University of Louisville

ThinkIR: The University of Louisville's Institutional Repository

Electronic Theses and Dissertations

$12-2018$

\title{
Characterizing the gut microbiota during plasmodium infection and antimalarial treatment.
}

Joshua E. Denny

University of Louisville

Follow this and additional works at: https://ir.library.louisville.edu/etd

Part of the Infectious Disease Commons

\section{Recommended Citation}

Denny, Joshua E., "Characterizing the gut microbiota during plasmodium infection and antimalarial treatment." (2018). Electronic Theses and Dissertations. Paper 3109.

https://doi.org/10.18297/etd/3109

This Doctoral Dissertation is brought to you for free and open access by ThinkIR: The University of Louisville's Institutional Repository. It has been accepted for inclusion in Electronic Theses and Dissertations by an authorized administrator of ThinkIR: The University of Louisville's Institutional Repository. This title appears here courtesy of the author, who has retained all other copyrights. For more information, please contact thinkir@louisville.edu. 
CHARACTERIZING THE GUT MICROBIOTA DURING PLASMODIUM INFECTION AND ANTIMALARIAL TREATMENT

\author{
By \\ Joshua E. Denny \\ B.S. Carson-Newman University, 2013
}

\begin{abstract}
A Dissertation
Submitted to the Faculty of the

School of Medicine of the University of Louisville

in Partial Fulfillment of the Requirements

for the Degree of
\end{abstract}

Doctor of Philosophy in Microbiology and Immunology

Department of Microbiology and Immunology

University of Louisville

Louisville, Kentucky

December 2018 

CHARACTERIZING THE GUT MICROBIOTA DURING PLASMODIUM INFECTION AND ANTIMALARIAL TREATMENT

By

Joshua E. Denny

B.S. Carson-Newman University, 2013

A Dissertation Approved on

November 9, 2018

by the following Dissertation Committee

Dr. Nathan W. Schmidt

Dr. Nejat Egilmez

Dr. Michele Kosiewicz

Dr. Yousef Abu Kwaik

Dr. Swati Joshi-Barve 


\section{DEDICATION}

\section{Dedicated to Faith}




\section{ACKNOWLEDGEMENTS}

My dissertation would not be complete without acknowledging all the people who helped me reach this point. First and foremost, of course, is Nathan, who let me join the lab despite my lack of experience with mice or malaria but also my lack of really any sort of clue about what I was doing. It is impossible to list all of the ways I am grateful to him for his role as my mentor, so instead I will simply say that as scientists we stand on the shoulders of the scientists that came before us, and there is no better or surer footing than I have found on Nathan's shoulders.

I am also indebted to the rest of the Schmidt lab over the years, to Nicolas, Sarah, Alivia, and briefly Chelsi and Bruce at UT, as well as Chris, Whitney, Morgan, and Rabindra here at UofL. From teaching me how to do things in the lab to making the lab a more enjoyable place, they all are irreplaceable.

My family has also been an essential source of support during my time as a grad student, and I would like to thank my parents and siblings for their encouragement. Also, now my dad can finally use his "pair o' docs"/paradox joke whenever we visit.

Finally, this list would be incomplete without my wife, Faith. She has supported me unconditionally throughout this process and all the difficulties it has 
entailed. I cannot thank her enough for her love and patience. She truly is my rock and my center. She deserves more words than I have space here to describe my gratitude at having her as my wife and partner during grad school. 


\author{
ABSTRACT \\ CHARACTERIZING THE GUT MICROBIOTA DURING PLASMODIUM \\ INFECTION AND ANTIMALARIAL TREATMENT \\ Joshua E. Denny
}

December 14, 2018

Plasmodium, the parasitic cause of malaria, is a global pathogen, annually causing 216 million infections and 445,000 deaths. As drug resistance continues develop and no effective vaccine is available, it is critical to understand the factors underlying the severity of this disease. Plasmodium is an extragastrointestinal tract infection where the parasite infects red blood cells causing clinical malaria. However, recent publications have pointed to interactions between the gut microbiota and malaria. With this in mind, the role of the gut microbiota in malaria infection was studied. C57BL/6 mice from different vendors displayed differential resistance and susceptibility to severe malaria, and cecal contents transplanted from these mice to germ-free mice recapitulated the observed phenotypes. Similarly, resistant mice possessed a much more robust humoral immune response than susceptible mice, which is critical for Plasmodium clearance. When the cecal contents from resistant and susceptible mice were sequenced, Lactobacillus and Bifidobacterium genera were enriched 
in resistant mice. Moreover, treating susceptible mice with probiotics containing these bacterial genera after antibiotic administration led to a lower parasite burden. These observations point to a previously unknown role for the microbiota in modulating the severity of malaria.

To further characterize the interactions between the host and gut microbiota in malaria, different components of gut homeostasis were investigated in both mild and severe disease. While intestinal permeability increased in both resistant and susceptible mice, there were no significant differences between the two groups. However, susceptible mice were shown to have greater numbers of lamina propria immune cells as well as greater abundances of cecal metabolites and bile acids during infection compared to resistant mice. Consistent with the decreased abundance of bile acids, histology showed much greater and prolonged damage and hemozoin deposition in the livers of susceptible mice compared to resistant mice. Despite these differences, the microbiota composition of resistant and susceptible mice became more similar during infection, although these changes were not associated with susceptibility or resistance when the altered cecal contents were transferred into germ-free mice. However, there were distinct differences in the functional capacity of the resistant and susceptible microbiota during infection. Susceptible mice showed significant increases in genes related to bacterial motility and flagellar assembly. Overall, there are profound differences in gut homeostasis during severe and mild Py infection. 
Finally, it was investigated whether antimalarial drugs, particularly clinically relevant artemisinin combination therapies (ACTs), could disrupt the gut microbiota. As previously shown, the composition of the gut microbiota alone can modulate the severity of Py infection; if ACTs change the microbiota composition, future infections could be more severe. To test this hypothesis, two common ACTs, artesunate plus amodiaquine and artemether plus lumefantrine, were used to orally treat mice while fecal pellets were collected to characterize the gut microbiota before and after treatment. After either ACT treatment, the overall species abundance in mice was similar to baseline. While alpha diversity remained unchanged by any treatment, there were minor, inconsistent changes in beta diversity that returned to baseline. With these findings, it does not appear that ACTs change the gut microbiota.

This work has greatly increased the scientific knowledge concerning the three-fold interaction between host, gut microbiota, and Plasmodium. While much work still needs to be done, these findings can provide a contextual foundation on which future work can be built. 


\title{
TABLE OF CONTENTS
}

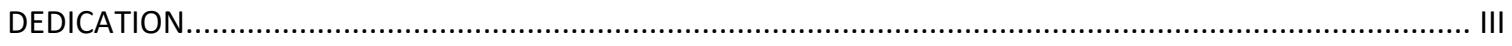

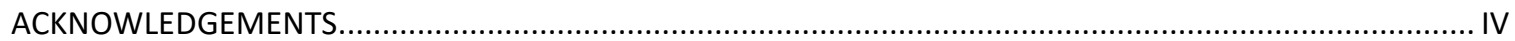

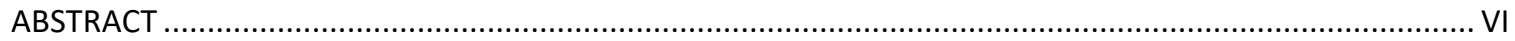

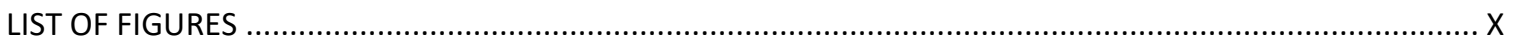

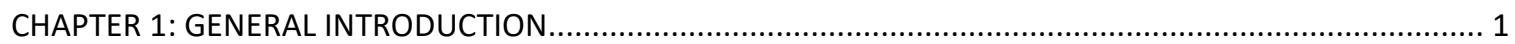 \\ CHAPTER 2: COMPOSITION OF THE GUT MICROBIOTA MODULATES THE SEVERITY OF MALARIA............. 16 \\ CHAPTER 3: SEVERITY OF PLASMODIUM INFECTION IN MICE DIFFERNTIALLY AFFECTS GUT-LIVER AXIS

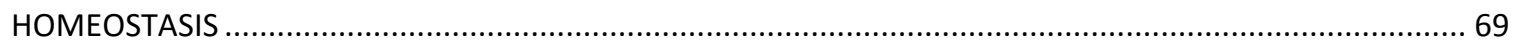 \\ CHAPTER 4: ORAL ADMINISTRATION OF CLINICALLY RELEVANT ANTIMALARIAL DRUGS DOES NOT MODIFY

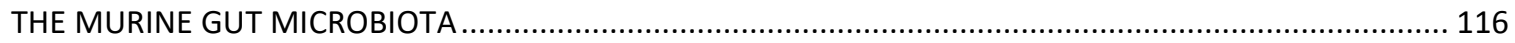 \\ CHAPTER 5: GENERAL DISCUSSION AND FUTURE DIRECTIONS ................................................ 130

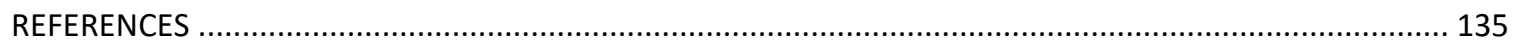

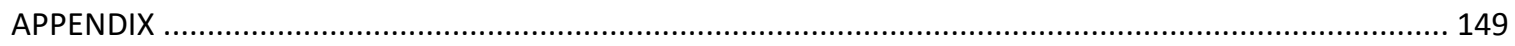

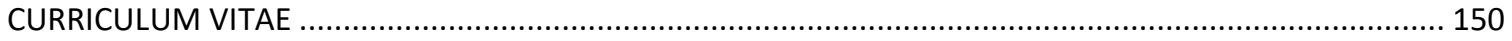




\section{LIST OF FIGURES}

Figure 2-1. Plasmodium parasite burden, morbidity and mortality vary by mouse vendor and diet.

Figure 2-2. Differential anemia and circulating parasitized red blood cells between mice from different vendors.

Figure 2-3. Similar susceptibility to parasitized red blood cell infection between C57BL/ 6

mice from different vendors.

Figure 2-4. C57BL/6 mice from Charles River are susceptible to high $P$. yoelii parasite burden.

Figure 2-5. Modulation of malaria pathogenesis by the gut microbiota is generalizable to another mouse strain and Plasmodium species.

Figure 2-6. Inversion of Jax and $\mathrm{NCl}$ in-house diets minimally effects severity of $P$. yoelii

infection.

Figure 2-7. Susceptibility to malaria correlates with differences in the gut microbial community structure.

Figure 2-8. Susceptibility to malaria correlates with differences in cecal bacteria populations.

Figure 2-9. Jackson mice fed Teklad 22/5 diet exhibit defined changes in cecal bacterial populations.

Figure 2-10. Distinct bacterial community structure in Jackson mice fed separate diets.

Figure 2-11. Changes in the gut microbial community structure correlate with changes in the gut and plasma metabolome in resistant and susceptible mice. ............................58

Figure 2-12. Variability in each sample type for PLS-DA. ........................................60

Figure 2-13. Gut microbiome shapes susceptibility to severe malaria. ............................61 Figure 2-14. Germ-free mice colonized with cecal content have similar bacterial diversity as donor sample.

Figure 2-15. Lactobacillus and Bifidobacterium are among the bacteria that drive differences between the gut-associated bacterial communities of resistant and susceptible mice.

Figure 2-16. Phylogenetic tree of bacteria isolated from stool samples and lab-cultured yogurt.

Figure 2-17. Treatment of gut microbiota intact mice with yogurt has a modest effect on parasite burden.

Figure 2-18. Susceptible mice treated with yogurt have decreased parasitemia and morbidity. .66 Figure 2-19. Resistant Jax mice have an elevated cellular and humoral immune response to Plasmodium. 
Figure 2-20. Gating strategy for T cell and B cell populations in Jax and $\mathrm{NCl}$ mice infected with $P$. yoelii.

Figure 3-1. Susceptibility to Plasmodium infection varies between vendors. C57BL/6N mice from Taconic (Tac) and Charles River (CR) were infected with P. yoelii (Py).........101 Figure 3-2. Small intestine lamina propria immune system changes during Py infection.

Figure 3-3. Gut bacterial community changes occurring post-Py infection do not change susceptibility to future Py infections .103

Figure 3-4. Metabolite profiles in selected tissues during Py infection. 105

Figure 3-5. Severity of liver damage following Py infection correlates with both parasitemia burden and kinetics. .106

Figure 3-6. Severity of Py infection correlates with loss of specific cecal bile acids......107 Figure 3-7. Severe Py infection increases the predicted functional capacity of the gut microbiota. 108

Figure 3-8. Schematic of the different host and microbiota factors..............................110

Figure 3-9. Gating strategies from the LP of mouse SI and LI.

Figure 3-10. Large intestine lamina propria immune system changes during Py infection.

Figure 3-11. The composition of gut bacteria populations changes after clearance of Py.

Figure 3-12. Small intestine bile acid profiles over Py infection.

Figure 3-13. Cecal bile acid profiles over Py infection .114

Figure 3-14. Plasma bile acid profiles over Py infection. .115

Figure 4-1. Relative bacterial abundances remain stable despite antimalarial treatments.

Figure 4-2. Antimalarial drug treatments cause minor changes to the diversity indices of the gut microbiota in mice. 


\section{CHAPTER 1}

\section{GENERAL INTRODUCTION}

\section{Plasmodium Life Cycle}

Malaria is a global health crisis, with approximately 2 million infections and 400,000 deaths annually, with the majority occurring in Africa in children under the age of $5^{1,2}$. Plasmodium, the causative agent of malaria, is a single-celled protozoan parasite with a multi-stage life cycle that occurs in the vertebrate host and the arthropod vector, generally a mosquito. In humans, the cycle begins when a female Anopheles mosquito takes a blood meal from a human host and injects Plasmodium sporozoites into the skin of the human host ${ }^{3}$. The sporozoites migrate to the bloodstream and are carried to the liver. At this stage, the infectious dose of sporozoites can be quite small; as few as 100 sporozoites can cause disease ${ }^{4}$. The sporozoites invade hepatocytes, where they differentiate into merozoites. This stage of disease is clinically silent with no symptoms of infection. Once the merozoites have matured, they exit the hepatocyte in membrane bound vesicles, called merosomes 5 . Upon rupture of the merosome membrane, merozoites enter the bloodstream where they will infect red blood cells (RBCs). Some Plasmodium species will infect differentially developed 
RBCs. For example, P. falciparum will infect RBCs at any developmental stage, while others, like $P$. vivax, will only infect immature reticulocytes 6 . Infection of RBCs has several steps: attachment, apical reorientation, junction formation, and invasion7. Initially, the merozoite must reach and attach to the RBC; once the merozoite is attached, it must reorient itself as the apical end must face the cell membrane for junction formation. Formation of the tight junction will "seal" the two cells together; as the merozoite moves into the RBC, the RBC membrane will fold around the merozoite to form the parasitophorous vacuole, where the parasite will reproduce ${ }^{8}$. At this point, most parasites will continue asexual reproduction, proliferating until lysing the $\mathrm{RBC}$ and infecting new RBCs. However, a few parasites will differentiate into gametocytes, which can be ingested by a mosquito, undergo sexual reproduction, and continue the Plasmodium life cycle in a new host ${ }^{8}$.

Plasmodium species can infect many different vertebrates, including humans, non-human primates, rodents, birds, and lizards ${ }^{9}$. The major Plasmodium species that cause morbidity and mortality in humans are $P$. falciparum $(\mathrm{Pf})$ and $P$. vivax $(\mathrm{Pv})$. Whereas $P$. ovale, $P$. knowlesi, and $P$. malariae also infect humans, they are of less clinical significance. Geographically, Plasmodium is restricted to tropical and subtropical regions due to distribution of mosquito vectors such as Anopheles gambiae and climate conditions with appropriate rainfall and temperature ${ }^{10}$. In areas with rainy seasons, malaria transmission is restricted seasonally as the mosquito vectors require standing 
water for development ${ }^{10}$. Generally, most human-specific Plasmodium species occupy the same geographic areas, and people can be infected with more than one species at a time ${ }^{3}$. However, $\mathrm{Pv}$ is restricted from sub-Saharan Africa due to the lack of the Duffy antigen receptor on RBCs in people of African descent; this receptor is required for $\mathrm{Pv}$ to invade $\mathrm{RBC} \mathbf{s}^{11}$. Due to this restriction, $\mathrm{Pf}$ is the dominant Plasmodium species in sub-Saharan Africa. One other significant difference between $\mathrm{Pf}$ and $\mathrm{Pv}$ is that $\mathrm{Pv}$ infection can relapse, which is the reemergence of merozoites after the primary parasite burden has been cleared ${ }^{12,13}$. During the Pv liver stage of infection, not all of the parasites differentiate into merozoites; some differentiate into hypnozoites that can remain dormant in the liver for months or even years after infection, the length of which can depend on the climate and the size of sporozoite inoculation ${ }^{14,15}$.

There are also several rodent-specific species of Plasmodium which are used as models for human malaria, the most common of which are $P$. berghei ANKA, $P$. yoelii, and $P$. chabaudi. Certain aspects of malaria infection are associated with specific mouse models. For example, cerebral malaria, which is one of the most lethal complications of infection, can be modeled by infecting C57BL/6 mice with Plasmodium berghei ANKA; approximately one week after infection, mice will begin to develop symptoms such as leaning, loss of balance, and paralysis followed by $100 \%$ mortality within 10 days of infection ${ }^{16}$. The mouse model recapitulates many of the mechanisms seen in human cerebral malaria, such as sequestration of parasitized RBCs (pRBCs) and localized 
inflammation ${ }^{17}$. The mechanism for these symptoms is discussed in more detail below. $P$. chabaudi infection, on the other hand, is generally non-lethal, and can model a more general human Plasmodium infection 6 . $P$. chabaudi, like Pf, infects RBCs at any stage of development and also reproduces many of the same pathological features as $\mathrm{Pf}$ or $\mathrm{Pv}$, such as anemia and splenomegaly as well as a similar immune response ${ }^{6}$. One interesting feature of $P$. chabaudi that is not present in other rodent models is recrudescence of blood stage parasite burden, although it is more limited compared to Pf. Finally, P. yoelii (Py) can be used to model the pathology and human immune response to $\mathrm{Pf}$, particularly the humoral response $^{18}$. Py has both lethal and non-lethal strains, $17 \mathrm{XL}$ and $17 \mathrm{XNL}$, respectively; the lethality of Py $17 \mathrm{XL}$ is multifactorial, but can include the induction of cerebral malaria and inflammation ${ }^{18,19}$. Mice infected with Py $17 \mathrm{XNL}$ develop sterilizing immunity to the parasite, and in this regard are considered good models for Pf vaccine development ${ }^{18}$.

\section{Host Response to Plasmodium Infection}

Clinically, Plasmodium infections are silent with no symptoms until the blood stage, where the parasite undergoes asexual replication. Once in the blood stage, common symptoms of uncomplicated malaria include fever and anemia; in complicated and severe cases, respiratory distress, coma or impaired consciousness, and severe anemia can be observed ${ }^{3}$. While the mortality rate for uncomplicated malaria is low, especially if treated with antimalarial drugs, the 
mortality rate for complicated severe malaria is estimated to be $90 \%$ if untreated and $20 \%$ if hospitalized 20,21 . However, the underlying pathology of these symptoms cannot be pinned solely on Plasmodium and its lytic behavior as host immunopathology has also been shown to play a role. During infection, Plasmodium causes a general inflammatory phenotype by inducing the production of inflammatory cytokines ${ }^{22}$. These cytokines, particularly tumor necrosis factor (TNF) family members, contribute to malaria symptoms, as administering recombinant TNF to naïve mice produces many of the same symptoms as malaria ${ }^{22}$. In a parallel process called sequestration, pRBCs will adhere to the host endothelium, particularly in the microvasculature; this can obstruct flow and lead to inflammatory cytokine and cell adhesion molecule (CAM) expression by the endothelial cells ${ }^{23,24}$. Several downstream effects of this response are increasing vascular permeability, leukocyte recruitment, and local ischemia and tissue damage ${ }^{23,24}$. Depending on the site of sequestration, the pathology will take different forms: sequestration in the intestinal microvasculature can lead to gastrointestinal symptoms such as diarrhea, while sequestration in the brain can lead to cerebral malaria, which is one of the more severe presentations and can lead to long-term neurological sequalae even after resolution of disease 23,25 .

While the immune system can be detrimental during malaria, it is critical for the resolution of infection. Both the innate and adaptive responses to Plasmodium play a role in resolution of blood-stage malaria. Natural killer (NK) 
cells produce interferon gamma (IFN $\gamma$ ) early in the infection, which correlates with resistance to malaria 26,27 . NK cell responses appear to be driven by IL-12 produced by other cell populations like monocytes and dendritic cells (DCs) ${ }^{28}$. While NK cells may not be the first to respond, they are early responders to Plasmodium infection and their activation correlates with protection.

Gamma delta T cells $(\mathrm{TCR} \gamma \delta)$ are innate-like T cell subsets that can have various functions depending on both differentiation and received signals, such as immunity to pathogens or tissue repair, and in many ways can act as a bridge between innate and adaptive immunity ${ }^{29}$. In some cases, $\operatorname{TCR} \gamma \delta$ cells can be the dominant producers of cytokines compared to CD4 or CD8 T cells ${ }^{30}$. During Plasmodium infection, a TCR $\gamma \delta$ subset can produce IFN $\gamma$ early in the infection to enhance the immune response, particularly differentiation and proliferation of germinal center $B$ cells and $T$ follicular helper cells $29,31,32$. Another subset can produce IL-17 in a Toll-like receptor-dependent manner, which can enhance the pathogen clearance and the humoral immune response in multiple infections, not just Plasmodium $30,33,34$.

Macrophages have dual roles in malaria infection, first acting as antigen presenting cells (APCs) but also phagocytosing infected RBCs and clearing them from circulation in a typically non-inflammatory fashion ${ }^{28}$. This occurs mainly through interactions with Plasmodium erythrocyte membrane protein 1 (EMP1) which is expressed on pRBCs and is anti-inflammatory35. However, macrophages can detect malarial DNA bound to hemozoin during phagocytosis, 
which will induce a strong pro-inflammatory response and can cause immune pathology36. Like macrophages, monocytes can also produce an antiinflammatory response to EMP135. However, it has also been shown that early after Plasmodium infection, monocytes infiltrate into the lamina propria of the intestine and cause inflammatory damage ${ }^{37,38}$. Plasmodium can also limit neutrophil populations, which can make patients susceptible to secondary infections ${ }^{39}$.

While these innate populations can in some ways bridge the innate and adaptive arms of the immune system during Plasmodium infection, dendritic cells (DCs) perform the majority of antigen presentation to T cells ${ }^{40}$. Generally, and unsurprisingly, dysregulation of DC antigen presentation is associated with morbidity and mortality during Plasmodium infection ${ }^{41}$. Studies looking at more particular aspects of DC function have been mixed and limited: for example, whether the parasite inhibits DC maturation. One study found in vitro that $\mathrm{pRBCs}$ bind to CD36 on DCs and inhibit MHCII expression and thus antigen presentation and $\mathrm{T}$ cell activation, while another study found that exposure to pRBCs did not affect the ability of DCs to promote a robust T cell response to malaria ${ }^{42,43}$. Despite conflicting data about the effect of malaria on DCs, antigen presentation to $T$ cells is critical for parasite clearance.

In particular, the humoral immune response is critical for clearance of blood-stage malaria ${ }^{44,45}$. To generate Plasmodium-specific antibodies, both T follicular helper (Tfh) cells and germinal center B (GC B) cells have to be 
activated and form germinal centers. Interactions between the Tfh cells and GC B cells lead to somatic hypermutation and class switching from $\lg M$ to $\lg G$, both of which enhance the antibody-mediated clearance of the parasite ${ }^{46}$. Common antibody targets of blood stage parasites include merozoite surface proteins 1 and 3 (MSP1 and MSP3) as well as apical membrane antigen 1 (AMA1), all of which are involved in parasite invasion of RBCs ${ }^{47,48}$. Trafficking of sporozoites from the dermis to the liver can be inhibited by sporozoite-specific antibodies against antigens such as circumsporozoite protein ${ }^{49}$. Antibodies can also target pRBCs themselves for opsonization and destruction by phagocytes, as well as inhibition of sequestration ${ }^{48}$. Like other aspects of anti-Plasmodium immunity, the parasite can modulate the host antibody response. It has been observed in humans that B cell memory is impaired during malaria infection, as antibody responses are short-lived and immunity to malaria is acquired gradually, particularly in areas of seasonal malaria transmission ${ }^{46,50}$. Both $\mathrm{T}$ and $\mathrm{B}$ cells can display "exhausted" phenotypes, characterized by a hypo-responsiveness to malaria and expression of PD-L1 and LAG3 on CD4 T cells ${ }^{51,52 . ~ I n ~ h u m a n s, ~ C D 4 ~}$ T cell expression of PD-L1 and LAG3 inversely correlates with B cell memory responses; this contributes to the difficulty in acquiring clinical immunity to malaria in areas with chronic Plasmodium exposure ${ }^{51,52}$. Blocking PD-L1 and LAG3 on T cells can reverse exhaustion and promote antibody-mediated Plasmodium clearance 51,52. Additionally, another mechanism Plasmodium can use to modulate humoral immunity in $P$. berghei ANKA infection in C57BL/6 mice 
is induction of a large IFN $\gamma$ and tumor necrosis factor-dependent inflammatory response to disrupt GC architecture and inhibit Tfh differentiation, both of which lead to an impaired humoral response ${ }^{53}$. Due to the prevalence of complicated malaria and the difficulty in achieving clinical immunity to Plasmodium, antimalarial drug treatments are essential for reducing malaria-associated morbidity and mortality.

\section{Treatment of Plasmodium Infections}

Frontline treatment of malaria is done with artemisinin combination therapies (ACTs). Artemisinin and its derivatives, artemether and artesunate, have a very rapid metabolism and half-life within the host; to complement this, longer-acting antimalarial drugs are combined to maximize the clinical efficacy the treatments. Two of the most common combinations are artemether paired with lumefantrine and artesunate paired with amodiaquine. ACTs are effective against the asexual stages of the Plasmodium life cycle, which translates clinically to the blood stage of infection. The World Health Organization recommends using the oral route of treatment, although ACTs can also be given intravenously, intramuscularly, or rectally ${ }^{20}$.

While ACTs have proven to be effective at treating malaria, the associated mechanisms of action for the drugs are unknown, to certain degrees. Lumefantrine and amodiaquine are thought to interfere with the ability of Plasmodium to metabolize heme, which is toxic to the parasite ${ }^{54}$. More is known 
about the mechanism of artemisinin and its derivatives. These drugs have been shown to be multifaceted in their effects on the host, but the major role these drugs appear to play is causing cellular damage and inhibiting proteasome function in Plasmodium 55 . This leads to the buildup of damaged proteins, endoplasmic reticulum stress, and induction of apoptosis, killing the parasite ${ }^{56}$. While artemisinin and derivatives have been relatively well characterized in their interactions with Plasmodium, much less is known about how artemisinin interacts with bacteria, particularly since the oral route is preferred for ACT administration. Artemisinin has been shown to kill Helicobacter pylori, but otherwise is not effective at killing various bacteria and fungi in vitro, such as Staphylococcus aureus or Bacillus subtilis57,58. Other tested artemisinin derivatives were shown to have moderate antibacterial and lesser antifungal properties, but most have not been approved for clinical use ${ }^{57}$.

\section{The Microbiota}

The microbiome is the collection of microbes and their genetic material associated with a host, and is typically delineated by occupied niche, such as skin and gut microbiome, while the microbiota refers to the collection of organisms. While bacteria have been the most widely studied, other organisms such as fungi and viruses are commensal as well. In the past, studying many of the microbiota constituents was out of reach due to their inability to be grown in lab conditions. However, the rise of $16 \mathrm{~S}$ rRNA sequencing has led to culture- 
independent methods of characterizing the microbiota. Variable regions within the $16 S$ rRNA gene are highly conserved between bacterial species and can be used to reliably identify bacterial taxa for phylogenetic analyses. Coupled with the low cost of DNA sequencing, the human and murine microbiomes have been well characterized, both in healthy conditions as well as in some disease states.

The gut microbiota can be influenced by many factors which can affect the composition or function of the microbiota, with two of the most studied being antibiotic treatment and diet. Given orally, antibiotics can act directly on the bacteria in the gut; given the right antibiotics and dosing, a majority of the microbiota can be depleted ${ }^{59}$. However, even subclinical doses of antibiotics can disrupt the microbiota and can contribute to the development of obesity or allergies 60,61 . Disruption of the microbiota can lead to disease as pathobionts in the gut such as Clostridium difficile or Enterococcus faecium can outgrow and establish niches ${ }^{59,62 .}$

The effect of host diet on the microbiota has also been studied extensively. Like antibiotics, shifts in diet can significantly reshape the microbiota. Adopting an animal-based diet, for example, can shift the microbiota composition to a more bile-tolerant community in as little as 48 hours $^{63}$. Diet-induced shifts in the microbiota can also be reversible. Different types of diet can have positive or negative effects, as different nutrients can be metabolized differently. For example, a high salt diet in mice has been shown to decrease Lactobacillus murinus in the gut; $L$. murinus can reduce Th17 cell numbers in the intestine and 
ameliorate experimental autoimmune encephalitis and salt-induced hypertension ${ }^{64}$. Conversely, a diet high in dietary fiber has been shown to be beneficial through fermentation and production of short-chain fatty acids (SCFAs) like butyrate and propionate 65 . SCFAs, particularly butyrate, have been shown to have many different effects on the host. Butyrate can induce an anti-inflammatory response in the intestine by acting as a histone deacetylase and regulating gene expression, primarily in macrophages and regulatory $T$ cells $(\text { Tregs })^{65}$. Modulation of the gut microbiota, through antibiotics or diet, has diverse effects on the host.

\section{The Gut Microbiota and Infectious Disease}

Due to the close nature of the relationship between the microbiota and the host, the microbiota has been investigated in infectious disease. As mentioned previously, $C$. difficile is a pathobiont in the gut microbiota and can take advantage of microbial and metabolic disruptions in the gut to proliferate ${ }^{66}$. Once C. difficile has established a niche in the host, it can cause abdominal pain and diarrhea67. While antibiotics can be used to treat acute $C$. difficile, recurrent infections can occur and antibiotic treatment can fail68. One overwhelmingly effective method of treating $C$. difficile infection is fecal microbiota transplantation (FMT) ${ }^{68}$. FMT is fairly straightforward, involving isolation of bacteria from donor feces and encapsulation for patient ingestion; a single treatment has been shown to resolve $C$. difficile infection in $70 \%$ of patients while that rate increases to $90 \%$ 
after a second FMT68. One reason for the success of FMT is the increase in overall bacterial diversity and abundance, particularly in the Bacteroides genus ${ }^{69}$. The increase in diversity can restore metabolic homeostasis in the gut, particularly secondary bile acid biosynthesis which confers resistance to $C$. difficile infection ${ }^{70}$. While $C$. difficile is an intestinal infection, the gut microbiota can also affect extra-intestinal infections as well.

One extra-intestinal disease that has been investigated is influenza virus, which despite infecting the respiratory tract, can be affected by the gut microbiota. Influenza can occasionally cause gastrointestinal symptoms like diarrhea. Treating mice with antibiotics has been shown to impair the ability of the host to form a sufficient virus-specific immune response to clear the virus ${ }^{71,72}$. Compared to untreated mice, antibiotic-treated mice have an impaired inflammatory response as well as significantly lower innate or adaptive virusspecific responses ${ }^{71,72}$. Conversely, influenza infection can change the composition of the microbiota, decreasing the abundance of Lactobacillus species and segmented filamentous bacteria but increasing the abundance of the Enterobacteriaceae family members and Sphingomonas species ${ }^{71,73}$. This dysbiosis was required for gastrointestinal symptoms. The dysbiosis, in turn, is caused by the expansion of inflammatory Th17 cells in the intestine that respond to the IFN $\gamma$ produced by lung-derived CD4+ T cells ${ }^{73}$. Overall, the gut microbiota is beneficial to the host, helping produce a robust anti-influenza immune response, but can itself be affected by influenza-induced inflammation. 
The microbiota has been shown to be important during Plasmodium infection. Members of the gut microbiota express the glycan Gala1-3Galß14GlcNAc-R (alpha-gal) on their surface, which induce anti-alpha-gal antibodies ${ }^{74}$. Plasmodium sporozoites, but not merozoites, also express alpha-gal on their surface, and the presence of anti-alpha-gal lgM antibodies correlates with moderate protection against malaria in humans ${ }^{74}$. Unlike humans, mice express alpha-gal; when alpha-gal deficient mice are orally treated with Escherichia coli O86:B7, which expresses alpha-gal, the mice develop anti-alpha-gal antibodies $^{74}$. While this antibody response did not affect disease severity, it inhibited Plasmodium sporozoite transmission, limiting the parasite's ability to reach the liver from its injection site in the $\operatorname{skin}^{74}$. Based on this data, the microbiota can provide protection from malaria through cross-reactivity of bacterial antigens.

Similarly, Plasmodium has been shown to cause intestinal pathology and affect the gut microbiota. 10 days after Py nigeriensis infection, there is considerable sequestration of pRBCs in the gut microvasculature, with significant infiltration of Ly6C+Ly6G- monocytes in the lamina propria along with an increase in inflammatory cytokine expression caused by the $\mathrm{pRBCs}^{37}$. During infection, a shift was seen in the microbiota composition at day 10 p.i., with an increase in Bacteroidetes and a decrease in Firmicutes; however, by day 15 p.i. the majority of the observed changes had returned to baseline ${ }^{37}$. When C57BL/6 mice are infected with $P$. berghei ANKA infection to model cerebral malaria, significant gut 
pathology occurs, including shortening of the intestines and villi along with increased mucus thickness and intestinal permeability 38 . Along with this pathology, the gut microbiota became increasingly different from baseline, with a decrease in abundance of the Lactobacillaceae family but increases in Enterobacteraceae and Verrucomicrobiaceae ${ }^{38}$. Overall, Plasmodium infections can cause inflammation-related damage to the intestine and modulate the composition of the gut microbiota.

With the convergence of malaria and the gut microbiota, the goal of this dissertation is to characterize interactions between the gut microbiota, the host, and Plasmodium using the mouse model of malaria. Additionally, the effect of antimalarial drugs on the composition of the gut microbiota is studied. 


\section{CHAPTER 2}

\section{COMPOSITION OF THE GUT MICROBIOTA MODULATES THE SEVERITY OF}

\section{MALARIA $^{1}$}

Plasmodium infections result in clinical presentations that range from asymptomatic to severe malaria resulting in approximately one million deaths annually. In spite of this, the factors that determine disease severity remain poorly understood. Here we show that the gut microbiota of mice influences the pathogenesis of malaria. Genetically similar mice from different commercial vendors, which exhibited differences in their gut bacterial community, had significant differences in parasite burden and mortality after infection with multiple Plasmodium species. Germ-free mice that received cecal content transplants from 'resistant' or 'susceptible' mice had low and high parasite burdens, respectively, demonstrating the gut microbiota shaped the severity of malaria. Among differences in the gut flora were increased abundances of Lactobacillus and Bifidobacterium in resistant mice. Susceptible mice treated with antibiotics

\footnotetext{
${ }^{1}$ Villarino, N. F. et al. Composition of the gut microbiota modulates the severity of malaria. Proceedings of the National Academy of Sciences of the United States of America 113, 2235-2240, doi:10.1073/pnas.1504887113 (2016).
} 
followed by yogurt made from these bacterial genera displayed a decreased parasite burden. Consistent with differences in parasite burden, resistant mice exhibited an elevated humoral immune response compared to susceptible mice. Collectively, these results identify the composition of the gut microbiota as a novel risk factor for severe malaria and modulation of the gut microbiota (e.g. probiotics) as a potential treatment to decrease parasite burden. 


\section{Introduction}

Plasmodium infections are a global health burden causing over 200 million cases of malaria and around one million deaths annually, with the vast majority of fatalities being children under the age of 5 living in sub-Saharan Africa ${ }^{75}$. Many Plasmodium infections are either asymptomatic or cause only mild malaria. Yet, some infections progress to severe malaria that most often manifest as impaired consciousness (cerebral malaria), respiratory distress, and severe anemia ${ }^{3}$. The best correlate of disease severity following $P$. falciparum infection in humans is parasite density 76,77 .

The gut microbiota impacts multiple facets of host physiology ${ }^{78}$ including shaping susceptibility to numerous diseases ${ }^{61,79-86}$. The effects of the gut microbiota on the host are strongly influenced by the collective composition of the bacterial populations ${ }^{87}$ and commensal flora are known to affect local pathogen burdens and host immunity88-90. In addition to influencing local gut immunity, the gut microbiome also affects host immunity to extra-gastrointestinal tract viral infections ${ }^{72}$.

Recent studies also support that the gut microbiome modulates Plasmodium infections in humans. Anti-a-gal antibodies, induced by the gut pathobiont E. coli O86:B7, cross-react with sporozoites from human and rodent Plasmodium species that impair transmission of the parasite between the vector and vertebrate host, however this cross-reactive immunity did not affect blood stage parasite burden ${ }^{74}$. Additionally, the stool bacteria composition of Malian 
children correlated prospectively with risk of $P$. falciparum infection, but not progression to febrile malaria91. Importantly, it remains unclear whether the gut microbiome also contributes to the development of severe malaria. Using a murine model of malaria, we demonstrate that the gut microbiome effects blood stage parasite burden and the subsequent severity of malaria.

\section{Results}

\section{Mice from Different Vendors Exhibit Differential Susceptibility to Malaria}

Genetically similar inbred strains of mice (C57BL/6) maintained by different vendors (Jackson Laboratories and Taconic) have differences in their gut bacterial communities ${ }^{92,93}$. To determine whether these differences had any effect on Plasmodium infections C57BL/6 mice from Jackson Laboratories (Jax), Taconic (Tac), National Cancer Institute/Charles River (NCl) and Harlan (Har) were infected with $P$. yoelii. Following infection, profound differences in parasitemia (the fraction of red blood cells infected with P. yoelii) were observed among the four groups of mice (Fig. 2-1A,B). Whereas resistant mice (Jax and Tac) exhibited a maximum of approximately $10 \%$ parasitemia, they had no signs of morbidity (weight loss) or mortality, which was in contrast to the substantial weight loss and mortality observed in susceptible mice ( $\mathrm{NCl}$ and Har) where parasitemia was $>60 \%$ (Fig. 2-1C,D). Moreover, $\mathrm{NCl}$ and Har mice exhibited more profound and longer lasting anemia (loss of red blood cells (RBCs) per $\mathrm{mL}$ ) compared to Jax and Tac mice (Fig. 2-2A). Additionally, when total number of 
RBCs per $\mathrm{mL}$ was used to derive total pathogen burden we noted similarities between the parasite burden as detected by parasitemia or parasitized RBCs (pRBCs) per $\mathrm{mL}$ of blood (Fig. 2-2B-E). Of note, mice infected with different doses of $P$. yoelii pRBCs showed similar parasitemia kinetics between the different doses and susceptibility to infection (Fig. 2-3) suggesting mice from different vendors are differentially susceptible to progression to severe malaria but not to blood stage infection.

To determine the broader applicability of these data, another mouse strain and two Plasmodium species were tested. BALB/c mice from Jax, Tac, Charles River (CR) and Har were infected with $P$. yoelii. Mice were purchased from $\mathrm{CR}$ in lieu of $\mathrm{NCl} / \mathrm{CR}$. Of note, $\mathrm{C} 57 \mathrm{BL} / 6$ mice purchased from $\mathrm{CR}$ exhibit similar parasitemia and morbidity as $\mathrm{NCl}$ mice following infection with $P$. yoelii (Fig. 2-4). Consistent with $P$. yoelii infections in C57BL/6 mice (Fig. 2-5), BALB/c mice from Jax and Tac exhibit reduced $P$. yoelii parasitemia compared to mice from $\mathrm{CR}$ and Har (Fig. 2-5A,B). Furthermore, C57BL/6 mice from Jax and Tac exhibited reduced parasitemia compared to $\mathrm{CR}$ and Har following Plasmodium chabaudi infection (Fig. 2-5C,D). Finally, we assessed the development of experimental cerebral malaria (ECM) in C57BL/6 mice infected with Plasmodium berghei ANKA. Jax and Tac mice trended towards reduced parasitemia compared to $\mathrm{NCl}$ and Har mice at early time points, moreover there was a significant $(p=0.04)$ difference in survival between these groups of mice (Fig. 2-5E,F). Collectively, these data indicate the severity of malaria is dependent on the source of mice. 
Diet is a strong modulator of organismal health as well as of the gut microbiome and its function ${ }^{63}$. To determine whether the diet could shape the severity of malaria, Jax and $\mathrm{NCI}$ mice were fed one of two commercially available rodent diets, either the in-house NIH-31 (used in Fig. 2-1A-D) or Teklad 22/5. Parasitemia in $\mathrm{NCl}$ mice was unaffected; however, Jax mice had high levels of parasitemia when fed Teklad 22/5 (Fig. 2-1E,F). Consistent with the parasitemia data, Jax mice fed Teklad 22/5 also exhibited substantial weight loss and elevated mortality compared to Jax mice fed NIH-31 (Fig. 2-1G,H). Since these diets had no effect on parasite burden in $\mathrm{NCl}$ mice, the changes in parasitemia in Jax mice were unlikely due to a direct effect of these diets on the parasite burden. Moreover, high parasite burdens in $\mathrm{NCl}$ mice fed $\mathrm{NIH}-31$, suggest this diet supported the proliferative expansion of $P$. yoelii. When Jax and $\mathrm{NCI}$ mice were placed on the reciprocal vendor-specific diet and then infected with $P$. yoelii we noted a modest increase in parasite burden in Jax mice fed the $\mathrm{NCl}$ in-house diet, but no effect of the Jax in-house diet on NCI mice (Fig. 2-6). Collectively, these data sets led to the hypothesis that the gut microbiota influenced Plasmodium infections.

\section{Gut Bacterial Community Structure and Function are Different in Resistant and Susceptible Mice}

To directly test for differences in the gut microbiome, sections of the gastrointestinal tract from resistant (Jax and Tac) and susceptible ( $\mathrm{NCl}$ and Har) 
mice were collected and the bacterial communities were characterized using $16 \mathrm{~S}$ rRNA gene analysis. There was a high degree of similarity between the microbial community assemblages found within the cecum and the colon of mice from the same vendor (Fig. 2-7A), while there were clear differences between the microbial communities of these regions compared to the distal half of the small intestine within the same vendor. Moreover, significant differences between mice from all vendors were apparent in the non-metric multidimensional scaling (NMDS) analysis of population structure within the cecum, with the susceptible $\mathrm{NCl}$ and Har libraries showing a comparative overlap with each other yet distinct differences when compared to the resistant Jax and Tac communities (Fig. 27B). Analysis of the cecal bacterial communities at the family level revealed substantial differences, with Clostridiaceae, Erysipelotrichaceae, Lactobacillaceae, and Peptostreptococcaceae (members of the Firmicutes phylum) being proportionally more abundant in resistant (Jax and Tac) mice, whereas Bacteroidaceae and Prevotellaceae (members of Bacteroidetes phylum), and Sutterellaceae (member of Proteobacteria phylum) were proportionally more abundant in susceptible ( $\mathrm{NCl}$ and Har) mice (Fig. 2-8A,B). Finally, dietary changes are capable of inducing significant changes in the gut microbiome ${ }^{94}$ that reach steady state within 3-4 days in mice ${ }^{95}$. Consistent with these reports, we observed defined changes in the gut bacterial communities in Jax mice fed Teklad 22/5 or NIH-31 (Fig. 2-9, 2-10), with a noted decrease in Peptostreptococcaceae in Jax mice fed Teklad shifting closer towards that seen 
in susceptible $\mathrm{NCl}$ and Har mice (Fig. 2-9C). These changes coincide with a shift in the severity of malaria between these two groups of mice (Fig. 2-1E-H).

Consistent with changes in the gut bacterial community, analysis of metabolites in the small intestine, cecum, and plasma of Jax and $\mathrm{NCl}$ mice revealed differential expression between each tissue (Fig. 2-11A). An F-test of partial least squared discriminant analysis (PLS-DA) ${ }^{96}$ used to probe variation between metabolite profiles in Jax and $\mathrm{NCI}$ mice on a per tissue basis confirmed that the means of the variate 1 , which differentiated Jax from $\mathrm{NCl}$ mice in all tissues, were significantly different $(p \leq 0.0003,0.0001,0.0001)$ for the small intestine, cecum, and plasma, respectively, (Fig. 2-11B-D, 2-12). Several metabolites exhibited large ( $\geq 1.5$ fold) and statistically significant $(p \leq 0.1)$ differences between Jax and $\mathrm{NCI}$ mice, with the top $25 \%$ of metabolites associated with distinct metabolic pathways (Fig. 2-11E,F). Collectively, differences in the gut bacterial populations and metabolites support the hypothesis that the severity of malaria is modulated by differences in gut bacterial communities.

\section{Differences in the Gut Microbiome Shape Susceptibility to Malaria}

To directly test this hypothesis, genetically identical germ-free (GF)

C57BL/6 mice received cecal content transplants from either Jax or $\mathrm{NCl}$ mice. Sequence analyses demonstrated the bacterial communities in colonized germfree mice reflected that of the donor communities and were different than the 
communities in germ-free mice exposed to only environmental microbes (Fig. 213A). Furthermore, there was only a slight decrease in community diversity between respective donor and colonized germ-free mice (Fig. 2-14). Following $P$. yoelii infection, germ-free mice that received either Jax or $\mathrm{NCl}$ cecal transplants had parasite burdens similar to control Jax and NCI mice (Fig. 2-13B,C). Both $\mathrm{NCl}$ control mice and germ-free mice that received $\mathrm{NCl}$ cecal transplants also had decreased survival compared to the Jax control mice and germ-free mice that received Jax cecal transplants (Fig. 2-13D). Collectively, these data provide a direct demonstration that the severity of malaria was modulated by the gut microbiota.

\section{Decreased Parasite Burden in Mice Treated with Lactobacillus and Bifidobacterium}

To identify individual microbial phylotypes that may shape the severity of malaria, a deeper analysis was performed on the bacterial communities in the cecum. When pooled by resistance (Jax/Tac) or susceptibility (NCl/Har) to $P$. yoelii, several phylotypes (referred to here as Operational Taxonomic Units, OTUs) emerged from a linear discriminant analysis (LDA) effect size (LEfSe)driven analysis ${ }^{97}$ as biomarkers of the resistant or susceptible phenotype. Among those differences, Lactobacillus and Bifidobacterium were overly abundant in the resistant mice compared to susceptible mice (Fig. 2-15), with differences in 
Lactobacillus being the greatest driver of the differential community structure between resistant and susceptible mice (Fig. 2-15B).

To evaluate the linkage between Lactobacillus and Bifidobacterium towards resistance to severe malaria, Jax and $\mathrm{NCI}$ mice were treated with lab-cultured yogurt supplemented with probiotics that contained Lactobacillus and Bifidobacterium species prior to and following infection with $P$. yoelii. DNA sequencing of Lactobacillus isolated from fecal pellets from Jax and $\mathrm{NCl}$ mice or lab-cultured yogurt demonstrated phylogenetic congruence (Fig. 2-16). Consumption of Lactobacillus and Bifidobacterium can modulate the gut microbial community structure ${ }^{98}$ or function ${ }^{99}$. Following infection with P. yoelii, both Jax and $\mathrm{NCl}$ mice treated with yogurt had a modest, but significant (Jax $p<0.0001 ; \mathrm{NCl} p=0.0418)$, decrease in parasite burden compared to control untreated mice (Fig. 2-17). Jax and $\mathrm{NCl}$ mice treated with milk used to make the yogurt showed a similar parasite burden as control Jax and NCI mice (mean

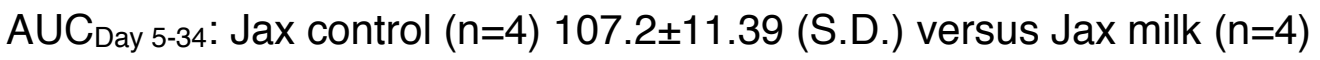
$83.55 \pm 24.83$ (S.D.) $p=0.13 ; \mathrm{NCl}$ control $(\mathrm{n}=4) 447.1 \pm 85.65$ (S.D.) versus $\mathrm{NCl}$ milk ( $n=3$ ) $384.8 \pm 73.08$ (S.D.) $p=0.36$ ). However, when mice were treated with antibiotics prior to yogurt treatment we observed a profound decrease in parasite burden in the susceptible $\mathrm{NCl}$ mice (Fig. 2-18A,B), and no weight loss in those mice compared to the other $\mathrm{NCl}$ groups (Fig. 2-18C). Whereas other constituents of the gut microbiota may contribute to regulating the severity of malaria, these 
data support the ability of Lactobacillus and Bifidobacterium to contribute towards the modulation of Plasmodium parasite burden.

\section{Severity of Malaria Tracks with the Magnitude of the Host Immune Response}

The gut microbiota can shape host immunity to systemic viral infections ${ }^{72}$ and T follicular helper (Tfh) produced IL-21 is required for GC B cell help and clearance of murine Plasmodium infections ${ }^{44}$. Consistent with these observations, resistant Jax mice exhibited an elevated $P$. yoelii-specific CD4+ T cell (CD49dhicD11ahi, Tfh cell, and GC B cell responses compared to susceptible $\mathrm{NCl}$ mice (Fig. 2-19A-C; Fig. 2-20) ${ }^{51}$. Jax and $\mathrm{NCl}$ mice had similar titers of IgM specific for the $19-\mathrm{kDa}$ fragment of merozoite surface protein $1\left(\mathrm{MSP} 1_{19}\right)$ from $P$. yoelii (Fig. 2-19D), suggesting similar activation of B cell in both groups. In contrast, Jax mice exhibited accelerated antibody class switching from MSP1 ${ }_{19-}$ specific IgM to IgG isotypes compared to NCl mice (Fig. 2-19D). These results support the possibility that the gut microbiome modulates the severity of malaria following $P$. yoelii infection through modulation of the host immune response.

\section{Discussion}

This study demonstrates that the murine gut microbiome influences the parasite burden of Plasmodium rodent species and modulates the severity of malaria in mice. Importantly, parasite burden is currently the best-known 
correlate of disease severity following $P$. falciparum infection in humans ${ }^{76,77}$. An association between the gut microbial community and Plasmodium parasites has been previously recognized in the mosquito vector ${ }^{100-104}$. Interestingly, the unique assemblage of skin bacteria on human skin has also been shown to impact the attractiveness of Anopheles mosquitoes to particular individuals ${ }^{105,106}$.

Two recent publications also support that the gut microbiota affects mammalian stages of the Plasmodium life cycle. The first study demonstrated that specific gut bacteria could impact the transmission of $P$. berghei sporozoites from mosquitos to mice ${ }^{74}$. The authors showed that the gut pathobiont, E. coli $\mathrm{O} 86: \mathrm{B} 7$, induced the production of anti-a-gal antibodies. When Plasmodium-infected mosquitoes injected sporozoites into the dermal tissue during a blood meal the anti-a-gal antibodies bound to the Plasmodium sporozoites, which prevented their migration to the liver ${ }^{74}$. These results also extended to humans where the presence of anti-a-gal IgM antibodies correlated with protection against $P$. falciparum infection. The effect of $E$. coli $\mathrm{O} 86: \mathrm{B} 7$ on Plasmodium infection was limited to transmission of sporozoites as there was no effect of the anti-a-gal antibodies on the symptomatic blood stage of the infection. Consistent with these findings a second report demonstrated that the unique composition of stool bacteria in Malian children correlated with prospective risk of $P$. falciparum infection, although not progression to febrile malaria ${ }^{91}$. Although the mechanism responsible for this observation is unknown, the similarities between these two studies (i.e., susceptibility to infection but not 
severity of blood stage infection) suggest the prospective risk of $P$. falciparum infection differentiated by stool bacteria composition may be attributed to differences in anti-a-gal IgM antibodies. In contrast to these two publications, we show that the gut microbiota modulates the severity of $P$. yoelii blood stage infections in mice, implying a novel and independent mechanism. Moreover, our findings show that the influence of the gut microbiome on Plasmodium infections is broad and not limited to the transmission of the parasite. Taken together our observation and those of Yilmaz et al. ${ }^{74}$, result in the intriguing speculation that the human intestinal microbiota might impact different stages of the Plasmodium life cycle in humans. Clearly, this is an area ripe for future research.

One potential mechanism by which the gut microbiota regulates the severity of malaria is a direct effect on the parasite itself where gut microbiotaderived products either promote or inhibit its growth. Whereas this possibility has not been formally excluded, we observe similar parasitemia expansion kinetics, when plotted on a log-scale, between days 5 and 11 post-infection in both resistant and susceptible mice. This observation suggests that the gut microbiota does not have a direct effect on the parasite. Consequently, it is more likely that the gut microbiota impacts the severity of malaria by modulating the host immune response to Plasmodium. Consistent with this possibility, resistant Jax mice exhibited an elevated anti-Plasmodium immune response compared to susceptible $\mathrm{NCI}$ mice. While these exciting data correlate with the parasite burden in these mice further experiments will be necessary to demonstrate the 
differential immune response is responsible for the difference in severity, and if so, how the gut microbiota modulates the host immune response to this extragastrointestinal infection. It has been previously shown that the gut microbiome provides signals to monocytes/macrophages that primed those cells to respond to and help control systemic LCMV infections ${ }^{72}$. Whether the gut microbiome modulates host immunity to Plasmodium through similar or different effects on the host immune system remains to be determined.

As mentioned above, diet has a major role in shaping the composition and activity of the gut microbiota94,107,108. Consequently, manipulating the structure and function of these complex communities through the diet provides an excellent opportunity to manipulate the host immune system ${ }^{107}$. In our study, we identified that Lactobacillus and Bifidobacterium species in cecal content could have a protective role by modulating the parasite burden and attenuating the severity of the disease. On the other hand, it is possible that these bacterial genera correlate with decreased parasitemia through niche competition that decreases the abundance of bacterial genera that cause elevated parasitemia. Since antibiotic treatment followed by yogurt treatment triggered a 14-fold reduction in parasite burden in susceptible mice, these exciting results suggest that through optimization (e.g., identifying and treating with the most effective 'protective' bacterial species or eliminating bacteria that contribute to high parasitemia) modulating the gut microbiome has the potential be a novel prophylaxis to prevent severe malaria. Consistent with this possibility, prior work 
has shown that children in a rural African village in Burkina Faso have an enrichment of the Bacteroidetes phylum and a depletion of the Firmicutes phylum, which contains Lactobacillus, compared to European children ${ }^{109}$. This resembles the community structure in susceptible mice that have increased Bacteroidetes and reduced Firmicutes compared to resistant mice (Fig. 2-15). Therefore, the commonality between the bacterial community structure in African children and Plasmodium susceptible mice suggests the possibility that probiotic modulation of the gut microbiota in mice to control severe malaria might work in humans.

This report demonstrates the novel observation that the severity of malaria in mice is profoundly impacted by the composition of the gut microbiota. The data lead to the hypothesis that differences in the gut microbiota may explain why some humans infected with Plasmodium progress to severe disease while others do not. The results also support the possibility that manipulating the gut microbiota has the potential to control the severity of malaria in humans. Whereas modulating the gut microbiota may not prevent Plasmodium infections, altering the gut microbiome has the potential to ameliorate severe disease and save hundreds of thousands of lives annually. 


\section{Materials and Methods}

\section{Animals and Housing}

Conventionally housed mice: Female C57BL/6J and BALB/cJ mice (6-8 weeks old) were purchased from The Jackson Laboratory (Bar Harbor, Maine);

C57BL/6N and BALB/cN mice (6-8 weeks old) were purchased from the National Cancer Institute (Frederick, MD), Charles River Laboratories (Wilmington, MD), Harlan Laboratories (Indianapolis, IN) and Taconic (Hudson, NY). Mice were acclimatized for a minimum of 7 days prior to starting experiments.

Germ-free mice: Female C57BL/6J mice (8-10 weeks old) were purchased from The National Gnotobiotic Rodent Resource Center at the University of North Carolina-Chapel Hill. Upon arrival at the University of Tennessee the mice were kept in the transporting box until fecal transplant was done, then mice were housed in conventional (specific pathogen free) conditions. Animal experiments were carried out at The University of Tennessee adhering to the local and national regulations of laboratory animal welfare. Procedures involving the care or use of mice were reviewed and approved by The University of Tennessee Institutional Animal Care and Use Committee.

Diets: Unless noted otherwise mice were fed NIH-31 Modified Open Formula Mouse/Rat Irradiated Diet (Harlan 7913) (Harlan, Indianapolis, IN). In some experiments mice were fed Teklad 22/5 Rodent Diet (Harlan 8640; Harlan, Indianapolis, IN), Jackson Laboratory in-house diet (5K67; Cincinnati Lab \& Pet 
Supply, Inc.; Cincinnati, OH) or NCI/CR in-house diet (5L79 Cincinnati Lab \& Pet

Supply, Inc.; Cincinnati, OH). Mice were placed on the respective diets upon arrival at the facility and maintained on the different diets for at least one week prior to P. yoelii infection or removal of intestinal tissues for bacterial population analysis.

\section{Plasmodium Infection}

Mice were infected with $P$. yoelii 17XNL, $P$. chabaudi AS, or P. berghei ANKA. For $P$. yoelii or $P$. berghei ANKA infections mice received $1 \times 10^{5}$ parasitized red blood cells (intravenously) prepared from frozen/thawed stabilites. For $P$. chabaudi infections, experimental mice were infected intraperitoneally with freshly prepared $1 \times 10^{5}$ parasitized red blood cells after one in vivo passage in C57BL/6 mice.

\section{Evaluation of Parasitemia}

Blood samples were taken from the tail at regular intervals from 3 to 35 days post-infection. Parasitemia was assessed by evaluation of thin blood smears or flow cytometry. Thin blood smears-at least 5 high-power (1000x) fields were assessed for each sample. Total red blood cells and parasitized red blood cells were counted in each field. Flow cytometry-about 5-10 $\mu \mathrm{l}$ of blood was added to PBS and then fixed with $0.00625 \%$ gluteraldehyde. Cells were stained with CD45.2-APC (clone 104; Biolegend; San Diego, CA), Ter119- 
APC/Cy7 (clone TER-119; Biolegend; San Diego, CA), dihydroethidium (Sigma Aldrich; St. Louis, MO), and Hoechst 33342 (Sigma Aldrich; St. Louis, MO). Red blood cells (CD45.2- Ter119+) were gated on and Plasmodium infected cells were subsequently identified as dihydroethium+ Hoechst 33342+. Parasitemia represents the percentage of red blood cells infected with $P$. yoelii, $P$. chabaudi or $P$. berghei.

\section{Enumeration of Red Blood Cells and Parasitized Red Blood Cells}

Blood samples were collected and used to quantify red blood cells per $\mathrm{mL}$ using a hemocytometer, and percent parasitemia. Red blood cells per $\mathrm{mL}$ and percent parasitemia were used to calculate the number of parasitized red blood cells per $\mathrm{mL}$ of blood.

\section{Cecum Transplant}

Cecal content was squeezed from donor mice ( $n=3-5$ mice per group) into a sterile petri dish. Immediately after harvesting the cecum material, it was diluted with sterile saline ( $2 \mathrm{~mL})$ and mixed gently for $\sim 30$ seconds. Each germfree mouse received the diluted cecum material ( $200 \mu \mathrm{L} /$ mouse) administered by oral gavage. For each mouse, a new sterile oral gavage needle was used. After the cecal transplant, mice were housed using conventional conditions. Mice were infected with $P$. yoelii 3 weeks after the cecal transplant. 


\section{Gut Microbiota Analysis}

Tissue collection: The distal half of the small intestine, cecum, and colon were excised from mice and flash frozen in liquid nitrogen. Samples were then stored at $-80^{\circ} \mathrm{C}$.

DNA isolation: DNA was extracted from samples using the MoBio PowerSoil DNA Isolation Kit (MoBio Laboratories Inc., Carlsbad, CA) according to manufacturer's protocols. No effort was made to separate mouse tissue from the bacterial component so as not to select against bacteria that were in close association with mouse tissue.

Ribosomal $16 S$ sequencing: For amplification of bacterial 16S rRNA genes, bacteria-specific PCR primers targeting the V4 region (bases 515-806) were used. Amplification, barcoding and sequencing were completed by the Genome Sequencing Center at the Hudson-Alpha Institute for Biotechnology (Huntsville, $A L)$ using the MiSeq platform with 150 bp paired-end reads.

Community analysis: We used the Mothur software package (version 1.33.1)110 to process sequences of sufficient length and quality similarly to the Schloss MiSeq SOP (http://www.mothur.org/wiki/MiSeq_SOP). Mothur was also used to cluster sequences into phylotypes and for phylogenetic classification and to sort our sequences into groups based on the region of the digestive tract from which the DNA was extracted. The Primer-E software package (Version 6) ${ }^{111}$ was used to interrogate the relationships between phylotypes across samples and to derive correlations between phylotype presence/abundance and other parameters. The 
“.shared" file (a matrix file containing phylotype abundances for each sample), created by Mothur, was imported directly into the Primer-E software package. All phylotype abundances were standardized to the total number of sequences per barcoded library (proportional abundances). Standardized abundances were square-root transformed to partially deemphasize more highly abundant phylotypes. A Bray-Curtis similarity matrix was constructed and used to perform non-metric multidimensional scaling analysis (NMDS) for visualization of community structure relationships between the different samples. Detection of "biomarker" sequences was performed using the software package LEfSe (http://huttenhower.sph.harvard.edu/galaxy/)97.

\section{Metabolomics Analysis}

Collection of small intestine and cecum content samples: Immediately after euthanasia, the abdominal cavity was exposed and the small intestine and the cecum were dissected from the other intestinal sections. The small intestine and cecum contents were collected into petri dishes by squeezing the tissues from the proximal to distal ends of the organs. In addition, extraction solvent $(1 \mathrm{~mL})$ (40:40:20 HPLC grade methanol, acetonitrile, water with $0.1 \%$ formic acid) was used to flush the small intestine and the cecum using a needle and syringe. The intestine contents were then transferred into separate cryotubes and flash frozen in liquid nitrogen. The samples were kept on ice for approximately one hour until extraction. 
Collection of plasma samples: Blood was collected from the peri-orbital sinus using heparinized capillary tubes under general anesthesia with isoflurane. Immediately after collection, blood samples were transferred to centrifuge tubes and centrifuged for 10 minutes at 2,000 $\mathrm{xg}$ at room temperature. Following centrifugation, plasma samples were transferred to cryotubes and flash frozen in liquid nitrogen. The samples were kept on ice for approximately one hour until extraction.

Sample extraction and analysis: Extraction of samples was allowed to proceed at $-78^{\circ} \mathrm{C}$ for 20 minutes. Samples were then centrifuged for 5 minutes (16.1 rcf) at $4^{\circ} \mathrm{C}$. The supernatant was transferred to new vials and the sample pellet was resuspended in $200 \mu \mathrm{L}$ of chilled $\left(4^{\circ} \mathrm{C}\right)$ extraction solvent. The extraction was allowed to proceed for $15 \mathrm{~min}$ at $-20^{\circ} \mathrm{C}$ at which time the samples were centrifuged for $5 \min (16.1 \mathrm{rcf})$ at $4^{\circ} \mathrm{C}$. The supernatant was transferred to vials and another $50 \mu \mathrm{L}$ of extraction solvent was added to the sample pellet where the extraction was repeated once more. Vials containing all of the collected supernatant were dried under a stream of $\mathrm{N}_{2}$ until the extraction solvent had evaporated. Solid residue was resuspended in $300 \mu \mathrm{L}$ of sterile water and transferred to autosampler vials. Samples were immediately placed in autosampler trays for mass spectrometric analysis.

Ultra-Performance Liquid Chromatograpy-High Resolution Mass Spectrometric (UPLC-MS) Analysis: Samples were placed in autosampler trays and kept at $4^{\circ} \mathrm{C}$. A $10 \mu \mathrm{L}$ aliquot of each was injected through a Synergi 2.5 micron Hydro- 
RP $100,100 \times 2.00 \mathrm{~mm} \mathrm{LC} \mathrm{column} \mathrm{(Phenomenex)} \mathrm{kept} \mathrm{at} 25^{\circ} \mathrm{C}$. The eluent was introduced into the MS via an electrospray ionization source conjoined to a Thermo Scientific Exactive Plus Orbitrap MS through a $0.1 \mathrm{~mm}$ internal diameter fused silica capillary tube, and the MS was run in fullscan mode with negative mode ionization using a method adapted from Rabinowitz ${ }^{112}$. Briefly, the samples were run with a spray voltage was $3 \mathrm{kV}$. The nitrogen sheath gas was set to a flow rate of $10 \mathrm{psi}$ with a capillary temperature of $320^{\circ} \mathrm{C}$. AGC target was set to 3e6. The samples were analyzed with a resolution of 140,000 and a scan window of 85 to $800 \mathrm{~m} / \mathrm{z}$ for from 0 to 9 minutes and 110 to $1000 \mathrm{~m} / \mathrm{z}$ from 9 to 25 minutes. Solvent A consisted of $97: 3$ water:methanol, $10 \mathrm{mM}$ tributylamine, and $15 \mathrm{mM}$ acetic acid. Solvent B was methanol. The gradient from 0 to 5 minutes was $0 \% \mathrm{~B}$, from 5 to 13 minutes was $20 \% \mathrm{~B}$, from 13 to 15.5 minutes was $55 \%$ $\mathrm{B}$, from 15.5 to 19 minutes was $95 \% \mathrm{~B}$, and from 19 to 25 minutes was $0 \% \mathrm{~B}$ with a flow rate of $200 \mu \mathrm{L} / \mathrm{min}$.

Metabolite feature extraction and data processing: The RAW files generated by the instrumental data collection software Xcalibur (Thermo Scientific) were converted to the mzML format ${ }^{113}$ using the ProteoWizard package ${ }^{114}$. The MAVEN software package (Princeton University) was used to automatically align the total ion chromatograms using the retention times of annotated metabolites and other dominant features from each sample ${ }^{115,116}$. Metabolites were manually identified and integrated using known masses ( $\pm 5 \mathrm{ppm}$ mass tolerance) and retention times ( $\Delta \leq 1.5 \mathrm{~min}$ ). Metabolite ion counts were normalized via mass for 
cecum and small intestine samples, and fold changes were calculated between samples. The resulting data were clustered using Cluster 3.0117 and heatmaps were then generated from clustered data using Java Treeview ${ }^{118}$ software. PCA analyses were performed and figures were generated using the statistical package $\mathrm{R}$ along with the ggplot2 ${ }^{119}$ and ggbiplot ${ }^{120}$ packages. PLS-DA plots were also generated via $\mathrm{R}$ along with the mixOmics ${ }^{121}$ package using metabolite areas as the predictors and mouse type as the discrete outcomes with a tolerance of $1 \times 10^{-6}$ and a max iteration of 500 .

\section{Yogurt treatment}

Antibiotic treated mice were treated orally with an antibiotic cocktail consisting of ampicillin $(0.5 \mathrm{mg} / \mathrm{ml})$ (Sigma Aldrich; St. Louis, MO), vancomycin $(0.25 \mathrm{mg} / \mathrm{ml})$ (Amnesco; Solon, $\mathrm{OH})$, metronidazole $(0.5 \mathrm{mg} / \mathrm{ml})$ (Spectrum Chemical Mfg. Comp.; Gardena, CA), neomycin sulfate $(0.5 \mathrm{mg} / \mathrm{ml})(\mathrm{EMD}$ Millipore; Billerica, MA), and gentamycin sulfate $(0.5 \mathrm{mg} / \mathrm{ml})$ (Corning; Manassas, VA). Mice were treated ad libitum for 3 weeks. Water bottles containing the antibiotic cocktail were changed weekly. After 3 weeks antibiotic treatment ceased and half of the antibiotic treated mice were then treated with yogurt for 3 weeks prior to the $P$. yoelii infection. Yogurt was made using a starter culture containing Lactobacillus bulgaricus, L. acidophilus, L. lactis, Bifidobacterium lactis, and Streptococcus thermophilus (Yogurt Starter Culture \#2, Custom Probiotics, Glendale, CA). In addition, yogurt was enriched with a probiotic 8 
powder supplement containing L. acidophilus, L. rhamnosus, L. salivarius, $L$. plantarum, L. casei, L. lactis, B. breve, B. infantis, B. longum, B. bifidum, and B. lactis (11 Strain Probiotic Powder, Custom Probiotics, Glendale, CA). Fresh yogurt was made for each treatment by adding the starter culture (1.2 grams) and the probiotic supplement (1.2 grams) to $15 \mathrm{~mL}$ of $2 \%$ reduced fat milk (Mayfield, Athens, TN) or organic $2 \%$ reduced fat milk-antibiotic pretreatment experiment (Organic Valley; La Farge, WI). The yogurt was then incubated at $37^{\circ} \mathrm{C}$ for $6-8$ hours before administration. Mice were treated with yogurt as described in figure legends with $0.2 \mathrm{ml}$ administered by oral gavage.

\section{Isolation and Sequencing of Lactobacillus}

Fecal pellets from Jackson and $\mathrm{NCl}$ mice were processed separately. Pellets were homogenized in buffered saline solution and plated onto Lactobacillus-selective MRS agar. Plates were subsequently incubated at $37^{\circ} \mathrm{C}$ in anaerobic culture jars. Single colonies were re-streaked three times to purify the strains. DNA was extracted from bacterial colonies, lab-cultured yogurt, and

probiotic powder using the MoBio Powersoil DNA extraction kit (MoBio, Carlsbad, CA) according to the manufacturer's protocols. An approximately $1500 \mathrm{bp}$ segment of the 16S rRNA gene was amplified from isolate DNA, via PCR, using the 9F-1522R primer set. PCR products were purified using the Qiaquick PCR purification kit (Qiagen, Valencia, CA) and sequenced at the University of Tennessee Molecular Biology Resource Facility using Sanger sequencing. 


\section{Cellular Immune Response}

Spleens were disrupted to generate single-cell suspensions in Hyclone RPMI 1640 media (Thermo Fisher Scientific Inc., Waltham, MA) supplemented with $10 \%$ fetal bovine serum (FBS) (Atlanta Biologicals, Inc., Lawrenceville, GA). RPMI 1640 was also supplemented with $1.19 \mathrm{mg} / \mathrm{ml}$ HEPES (Thermo Fisher Scientific Inc., Waltham, MA), 0.2 mg/ml L-glutamine (Research Products International Corp., Mt. Prospect, IL), (0.05 units $/ \mathrm{ml} \& 0.05 \mathrm{mg} / \mathrm{ml})$ penicillin/streptomycin (Invitrogen, Grand Island, NY), $0.05 \mathrm{mg} / \mathrm{ml}$ gentamicin sulfate (Invitrogen, Grand Island, NY), and $0.05 \mu \mathrm{M}$ 2-Mercaptoethanol (Thermo Fisher Scientific Inc., Waltham, MA). Single cell suspensions were treated with ammonium chloride potassium to lyse red blood cells. Spleens were harvested as indicated at the number of days post infection. Cells were stained with Fc block (anti-CD16/32; clone 2.4G2) and the following fluorescence-conjugated antibodies (CD45.2; clone 104, CD4; clone RM4-5, CD8; clone 53-6.7, CD3; clone 17A2, CD19; clone 6D5, Ter119; clone Ter-119, CD11a; clone M17/4, CD49d; clone R1-2, PD-1; clone 29F.1A12, CD95; clone Jo2, GL7; clone GL7, biotion-CXCR5; clone 2G8, CD44; clone IM7) purchased from Biolegend (San Diego, CA) and BD Biosciences (San Diego, CA). For CXCR5 staining cells were stained with biotinylated-CXCR5 for 30 minutes at room temperature prior to staining with fluorescence-conjugated streptavidin. Antibodies were resuspended in FACS buffer (1x PBS, 1\% FBS, $0.02 \%$ sodium azide) and cells were stained 
for 30 minutes at $4^{\circ} \mathrm{C}$. Following staining cells were fixed and permeabilized with Fixation Buffer (Biolegend, San Diego, California). Cells were acquired through an LSR II (BD Biosciences). Data were analyzed by FlowJo software (Tree Star, Ashland, OR).

\section{Detection of $P$. yoelii MSP1 ${ }_{19}$-specific Antibodies}

Serum was collected on the indicated days post- $P$. yoelii infection and stored at $-20^{\circ} \mathrm{C}$. MaxiSorp Immuno plates (Thermo Scientific) were coated with 1 $\mu \mathrm{g} / \mathrm{ml}$ recombinant MSP1 ${ }_{19}$ (The following reagent was obtained through the MR4 as part of the BEI Resources Repository, NIAID, NIH: Plasmodium yoelii yP.y.MSP1-19(XL)/VQ1, MRA-48, deposited by DC Kaslow.) Dilutions of serum were added to wells. Total MSP1 ${ }_{19}$-specific $\lg M$, IgG1, $\lg G 2 b, \lg G 2 c$, and $\lg G 3$ antibodies were detected with horseradish peroxidase-conjugated goat antimouse $\lg M$, IgG1, IgG2b, IgG2c, and IgG3, respectively, (Jackson ImmunoResearch) and 3,3',5,5'-tetramethylbenzidine substrate (Arcos Organics). Reactions were stopped by addition of $2 \mathrm{M} \mathrm{H}_{2} \mathrm{SO}_{4}$. Results are presented as average endpoint titers with absorbance readings below 0.1 (absorbance at $450 \mathrm{~nm}$ ).

\section{Statistical Analysis}

Descriptive and comparative statistical analyses of data, except the gut microbiota and metabolomics data, were done using GraphPad Software version 
6 (La Jolla, CA, USA). The area under the parasitemia curve (AUC) was estimated for each group following the trapezoidal rule with the following equation ${ }^{122,123:}$

$$
A U C_{\text {t1-t-last }}=0.5 \Sigma\left(Y_{i}+Y_{i+1}\right){ }^{*}\left(t_{i+1}-t_{i}\right)
$$

where "t" was sampling time and " $y$ " the observed outcome (e.g., \%parasitemia). 
Figures and Figure Legends
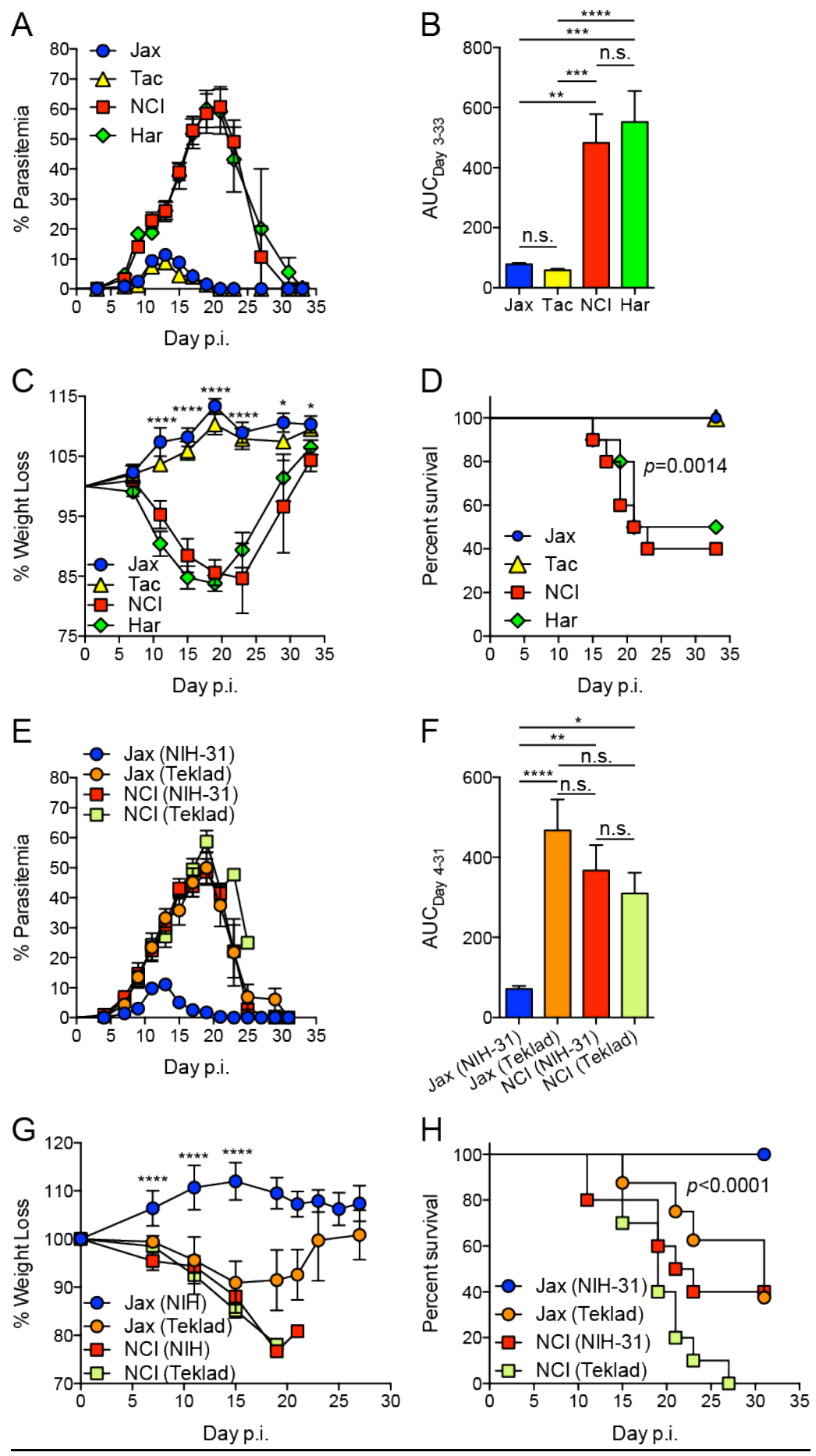

Figure 2-1. Plasmodium parasite burden, morbidity and mortality vary by mouse vendor and diet. C57BL/6 mice were infected with $P$. yoelii parasitized 
red blood cells. A. Fraction of red blood cells infected with $P$. yoelii

(\%parasitemia). B. Area under the parasitemia curve (AUC) analysis. Data were analyzed by one-way ANOVA and Tukey's multiple comparison post-test. C. Percent weight loss following infection. Data were analyzed by one-way ANOVA. D. Survival of mice following infection. Survival curves were analyzed by Logrank (Mantel-Cox) test. E-H. Mice were fed either NIH-31 or Teklad 22/5 diets before and after $P$. yoelii infection. E. Percent parasitemia following $P$. yoelii infection. F. AUC analysis. Data were analyzed by one-way ANOVA and Tukey's multiple comparison post-test. G. Percent weight loss following infection. Data were analyzed by one-way ANOVA. H. Survival of mice following infection. Survival curves were analyzed by Log-rank (Mantel-Cox) test. A-F, H. Data (mean \pm S.E.) are cumulative results ( $n=8-10$ mice/group) from two experiments. G. Data (mean \pm S.D.) are from 4-5 mice per group from one experiment. * $p<$ $0.05,{ }^{* \star} p<0.01,{ }^{* \star *} p<0.001,{ }^{* \star * *} p<0.0001$, n.s. $=$ not significant. 

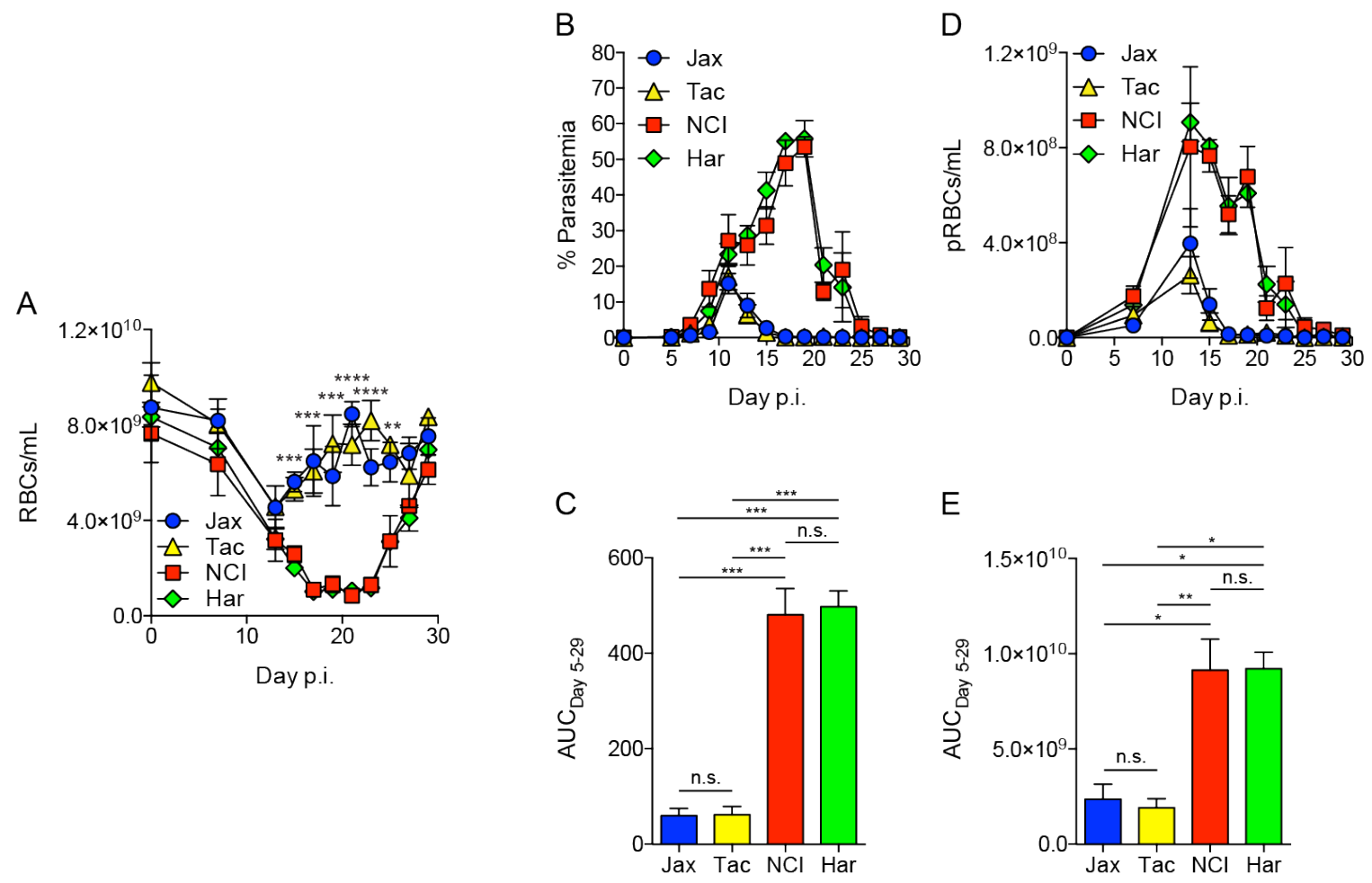

Figure 2-2. Differential anemia and circulating parasitized red blood cells between mice from different vendors. C57BL/6 mice were infected with $1 \times 10^{5}$ P. yoelii parasitized red blood cells. A. Number of red blood cells (RBCs) per mL of blood. B. Fraction of red blood cells infected with P. yoelii (\%parasitemia). C. Percent parasitemia AUC analysis. D. Number of parasitized red blood cells (pRBCs) per $\mathrm{mL}$ of blood. E. pRBCs/mL of blood AUC analysis. A-E. Data (mean \pm S.E.) are from 3-4 mice/group. A, C, and E. Data were analyzed by oneway ANOVA and Tukey's multiple comparison post-test. ${ }^{*} p<0.05,{ }^{* *} p<0.01$, ${ }^{* * *} p<0.001,{ }^{* * * *} p<0.0001$, n.s. $=$ not significant. 

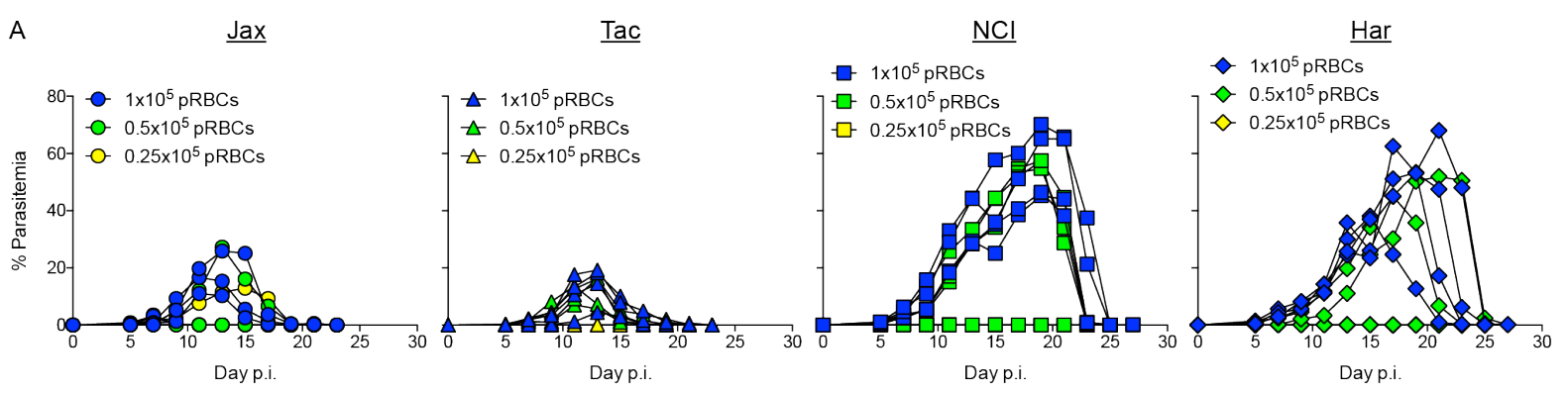

$\begin{array}{lccccc}\text { B } & \begin{array}{c}\mathrm{Jax} \\ \text { Infected/Total }\end{array} & \begin{array}{c}\text { Tac } \\ \text { Infected/Total }\end{array} & \begin{array}{c}\underline{\mathrm{NCl}} \\ \text { Infected/Total }\end{array} & \begin{array}{c}\underline{\mathrm{Har}} \\ \text { Infected/Total }\end{array} & \underline{\text { Fischer's Exact Test }} \\ 1.00 \times 10^{5} \mathrm{pRBCs} & 7 / 8 & 8 / 8 & 8 / 8 & 6 / 8 & 6 / 8 \text { vs. } 8 / 8 p=0.47 \\ 0.50 \times 10^{5} \mathrm{pRBCs} & 5 / 8 & 6 / 8 & 4 / 8 & 3 / 8 & 3 / 8 \text { vs. } 6 / 8 p=0.31 \\ 0.25 \times 10^{5} \mathrm{pRBCs} & 2 / 8 & 2 / 8 & 0 / 8 & 0 / 8 & 0 / 8 \text { vs. } 2 / 8 p=0.47\end{array}$

Figure 2-3. Similar susceptibility to parasitized red blood cell infection between C57BL/6 mice from different vendors. C57BL/6 mice from the indicated vendors were infected with the indicated number of $P$. yoelii parasitized red blood cells (pRBCs). A. Fraction of RBCs infected with P. yoelii (\%parasitemia). Each line represents parasitemia kinetics from an individual mouse ( $n=4$ mice/dose/vendor). Data are representative of two experiments. B. Number of mice from each vendor infected with the indicated dose of pRBCs and the total number of mice injected. Data are cumulative results from two experiments. Susceptibility to infection at the different doses between vendors was analyzed by two-tailed Fisher's Exact Test. 

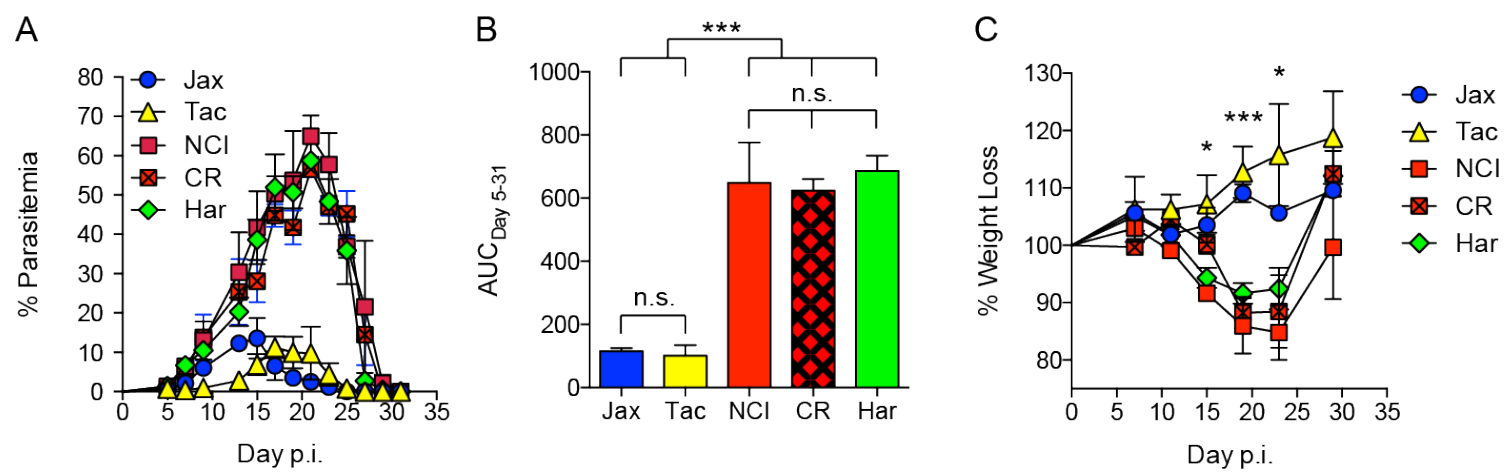

Figure 2-4. C57BL/6 mice from Charles River are susceptible to high $P$. yoelii parasite burden. C57BL/6 mice were infected with $1 \times 10^{5} P$. yoelii parasitized red blood cells. A. Fraction of red blood cells infected with $P$. yoelii (\%parasitemia). B. Area under the parasitemia curve (AUC) analysis. Data were analyzed by one-way ANOVA and Tukey's multiple comparison test. C. Percent weight loss following infection. Data were analyzed by one-way ANOVA. A-C. Data (mean \pm S.E.) are from 3-5 mice/group and representative of two experiments. ${ }^{*} p<0.05,{ }^{* * *} p<0.001$, n.s. $=$ not significant. 


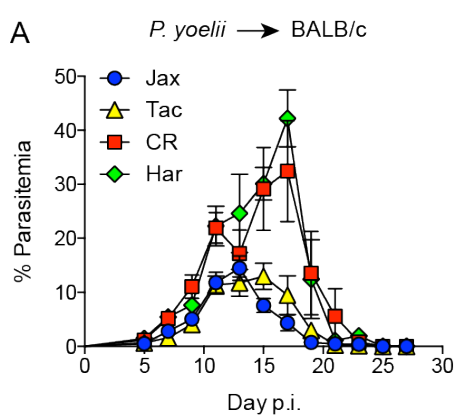

B

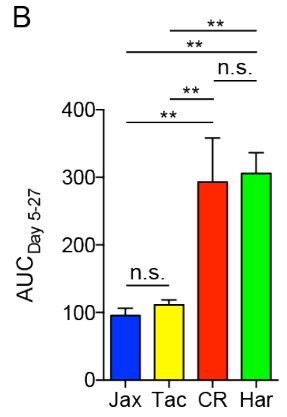

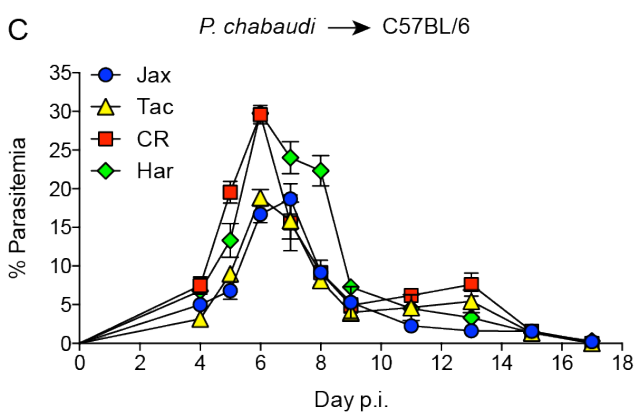

D

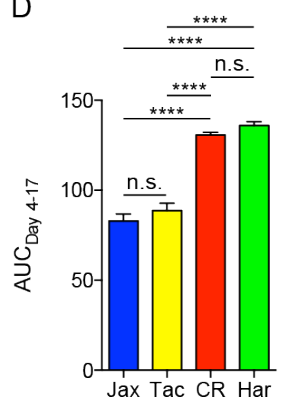

E P. bergheiANKA $\rightarrow$ C57BL/6

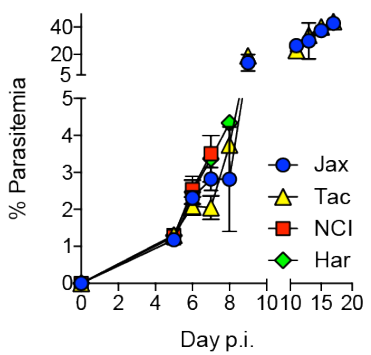

$\mathrm{F}$

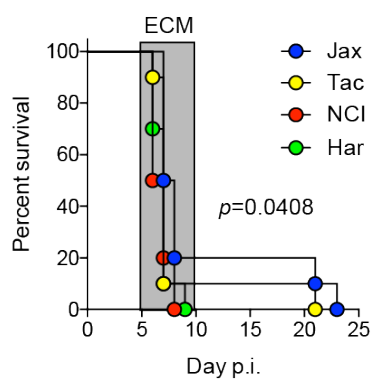

Figure 2-5. Modulation of malaria pathogenesis by the gut microbiota is generalizable to another mouse strain and Plasmodium species. A-B. BALB/c mice were infected with $1 \times 10^{5} P$. yoelii parasitized red blood cells. A. Fraction of red blood cells infected with $P$. yoelii (\%parasitemia). B. Area under the parasitemia curve (AUC) analysis. Data were analyzed by one-way ANOVA and Tukey's multiple comparison test. A-B. Data (mean \pm S.E.) are cumulative results ( $\mathrm{n}=8$ mice/group) from two experiments. C-D. C57BL/6 mice were infected with $1 \times 10^{5}$ P. chabaudi parasitized red blood cells. C. Fraction of red blood cells infected with $P$. chabaudi (\%parasitemia). D. Area under the parasitemia curve (AUC) analysis. Data were analyzed by one-way ANOVA and Tukey’s multiple comparison test. C-D. Data (mean \pm S.E.) are from 3-4 mice/group and representative of two experiments. E-F. C57BL/6 mice were infected with $1 \times 10^{5} P$. berghei ANKA parasitized red blood cells. E. Fraction of 
red blood cells infected with $P$. berghei (\%parasitemia) from surviving mice. Data (mean \pm S.E.) are cumulative results ( $n=10$ mice/group) from two experiments. $\mathbf{F}$. Survival of mice following infection ( $n=10$ mice/group). Survival curves were analyzed by Log-rank (Mantel-Cox) test. ${ }^{\star *} p<0.01,{ }^{\star \star \star \star} p<0.0001$, n.s. $=$ not significant.
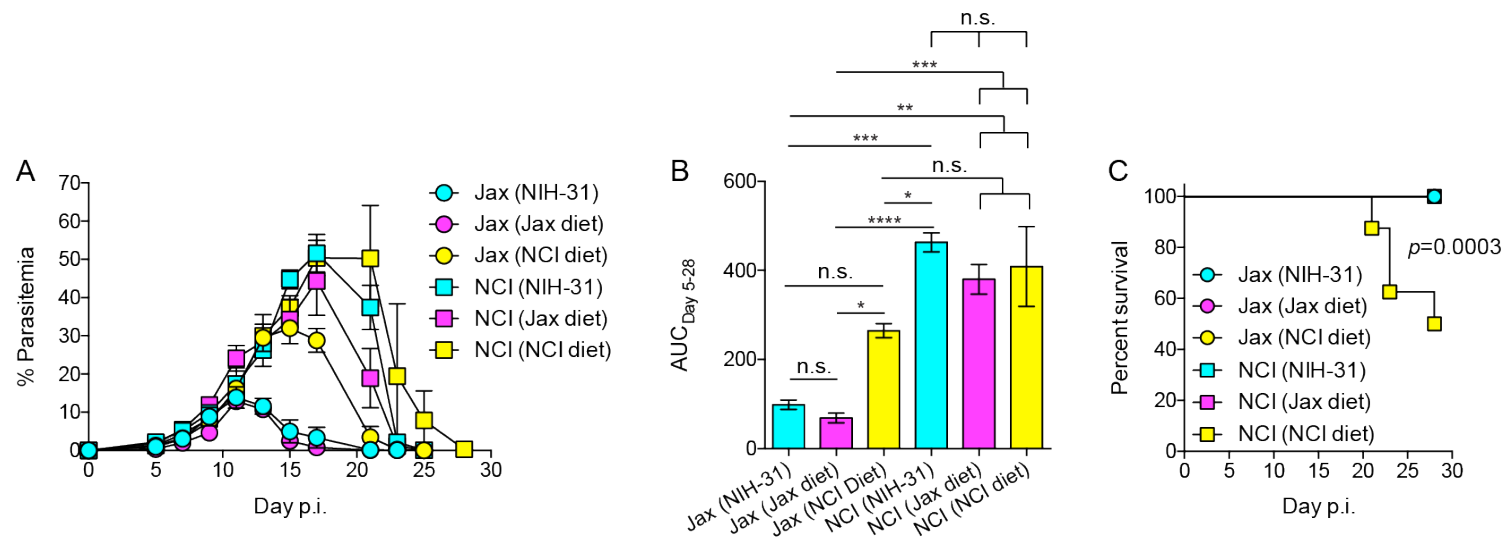

Figure 2-6. Inversion of Jax and NCl in-house diets minimally effects severity of $\boldsymbol{P}$. yoelii infection. C57BL/6 mice were placed on one of three different rodent chows; $\mathrm{NIH}-31$, Jax in-house diet, or $\mathrm{NCl}$ in-house diet, for oneweek prior to infection through resolution of infection. Mice were infected with $1 \times 10^{5}$ P. yoelii parasitized red blood cells. A. Fraction of red blood cells infected with P. yoelii (\%parasitemia). B. Area under the parasitemia curve (AUC) analysis. A-B. Data (mean \pm S.E.) are from 3-4 mice/group and representative of two experiments. ${ }^{*} p<0.05,{ }^{* *} p<0.01,{ }^{* * *} p<0.001,{ }^{* * *} p<0.0001$, n.s. $=$ not significant. C. Survival of mice following infection ( $n=7-8$ mice/group) from two experiments. Survival curves were analyzed by Log-rank (Mantel-Cox) test. 

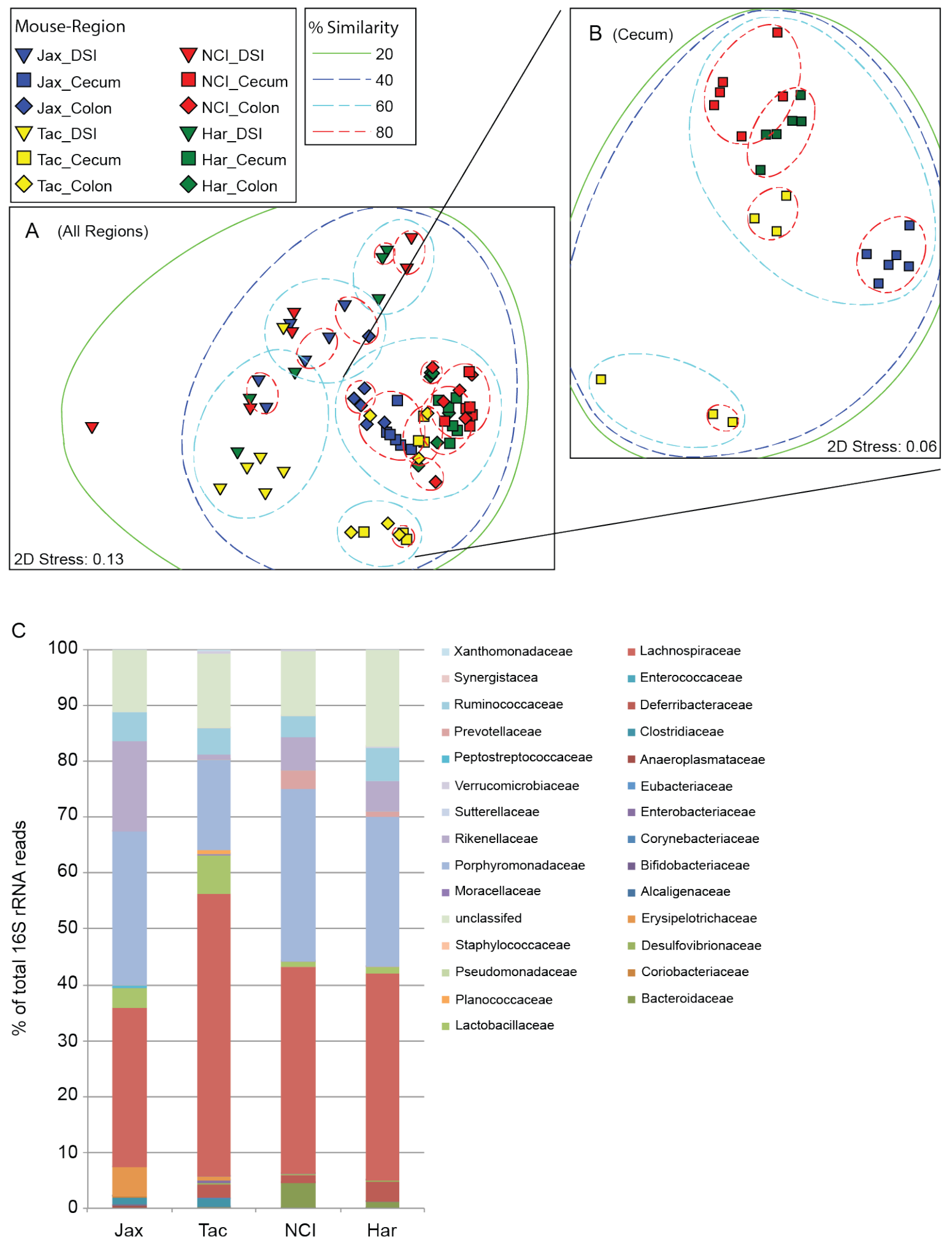
Figure 2-7. Susceptibility to malaria correlates with differences in the gut microbial community structure. Bacterial population analysis was performed using nonmetric multidimensional scaling analysis. The closer together two points are the more similar the libraries are to one another. Libraries with similarities greater than $20 \%, 40 \%, 60 \%$ or $80 \%$ are encircled with green, blue, teal or red dotted lines, respectively. Triangle=distal portion of the small intestine (DSI); Square=cecum; Diamond=colon. A. Analysis of all three regions of the digestive tract. B. Analysis of only the cecum bacterial populations. C. Percentage of $16 \mathrm{~S}$ rRNA gene reads of bacterial families comprising at least $0.01 \%$ of reads from at least 1 sample. Abundances of $16 S$ reads at the Family level were first normalized to proportional abundances and then the average proportional abundance $(n=6)$ for each family was calculated for mice from each of the four vendors. 

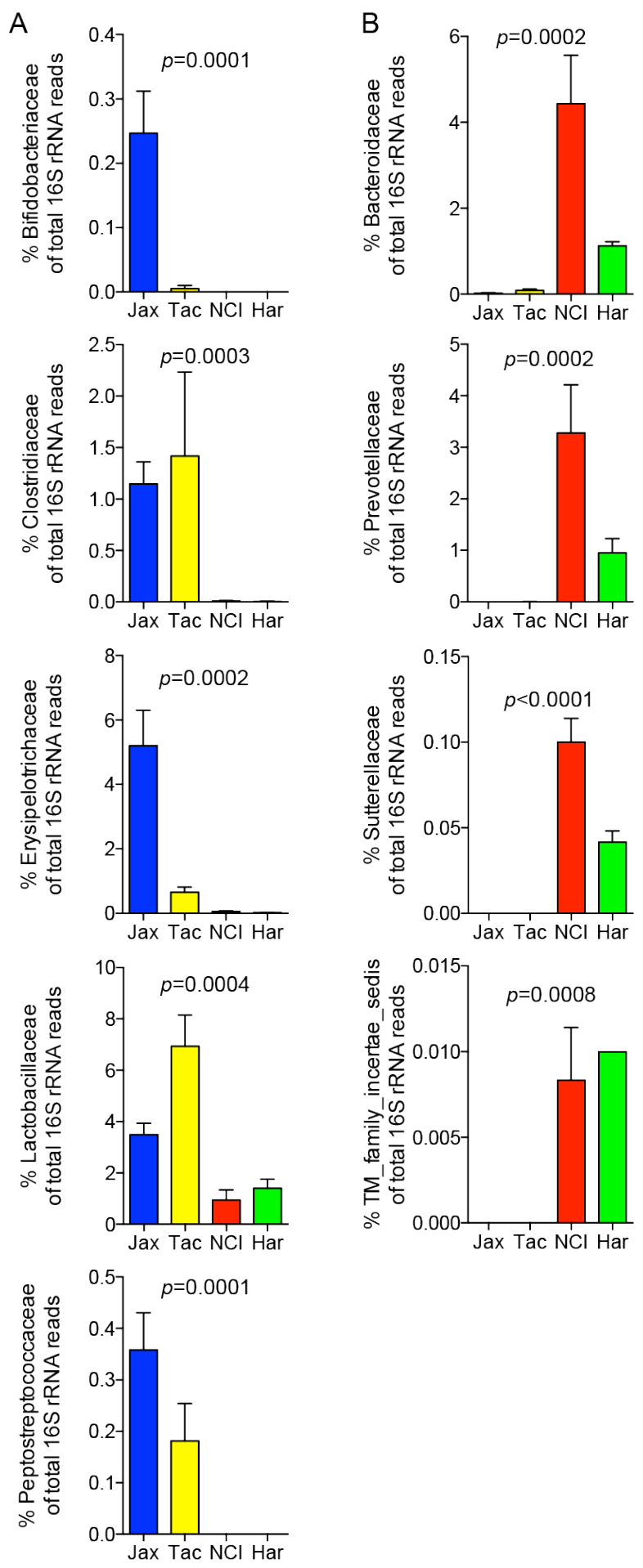

Figure 2-8. Susceptibility to malaria correlates with differences in cecal bacteria populations. A. Bacterial families that were identified as being significantly enriched in Jackson or Taconic mice. B. Bacterial families identified 
as being significantly enriched in $\mathrm{NCl}$ or Harlan mice. A-B. Data (mean \pm S.E.) are from 6 mice per group and extracted from analysis in Fig. 2-11c. Data were analyzed by Kruskal-Wallis test.
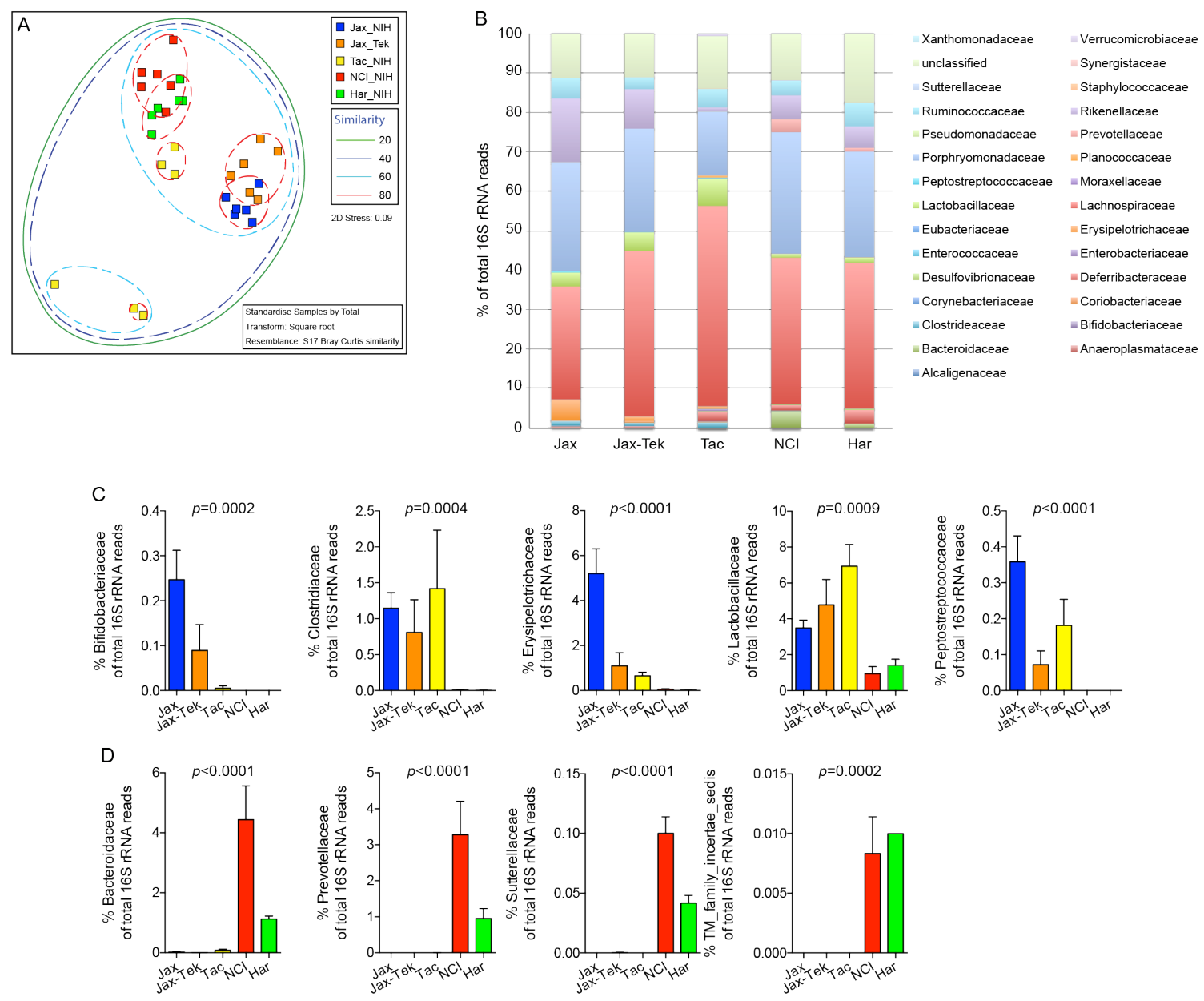

Figure 2-9. Jackson mice fed Teklad 22/5 diet exhibit defined changes in cecal bacterial populations. All mice were fed $\mathrm{NIH}-31$, except one group of Jackson mice that received Teklad 22/5 (Jax-Tek). A. Cecal bacterial population analysis was performed using NMDS. The closer together two points are the more similar the libraries are to one another. Libraries with similarities greater 
than $20 \%, 40 \%, 60 \%$ or $80 \%$ are encircled with green, blue, teal or red dotted lines, respectively. Analysis of cecal bacterial populations. Each symbol represents an individual mouse. B. Percentage of $16 \mathrm{~S}$ rRNA gene reads of bacterial families comprising at least $0.01 \%$ of reads from at least 1 sample. Abundances of $16 \mathrm{~S}$ reads at the Family level were first normalized to proportional abundances and then the average proportional abundance $(n=6)$ for each family was calculated for mice from each of the groups. C. Bacterial families that were identified as being significantly enriched in Jackson or Taconic mice. D. Bacterial families identified as being significantly enriched in $\mathrm{NCl}$ or Harlan mice. C-D. Data (mean \pm S.E.) are from 6 mice per group and extracted from analysis in panel B. Data were analyzed by Kruskal-Wallis test. 


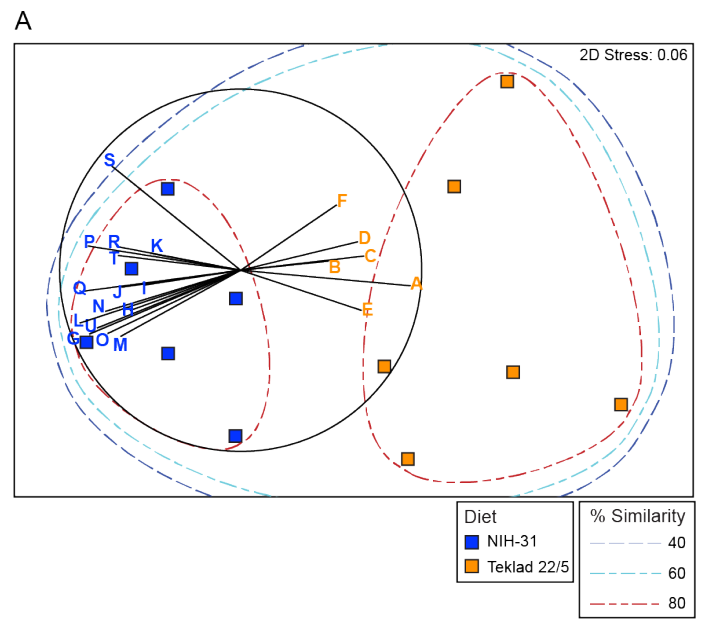

B

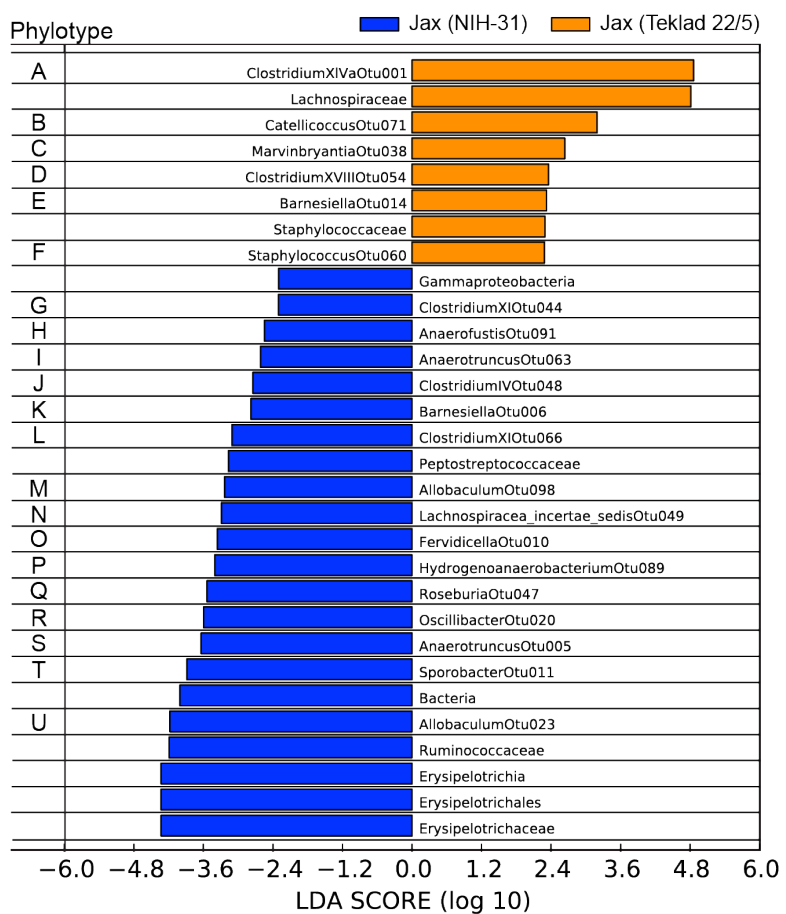

Figure 2-10. Distinct bacterial community structure in Jackson mice fed separate diets. C57BL/6 mice from Jackson Laboratory (Jax) were fed two separate diets $(\mathrm{NIH}-31(\mathrm{n}=6)$ or Teklad $22 / 5(n=6))$ for one to three weeks prior to removal of gut contents and analysis of gut bacterial community. A. NMDS plot displaying the similarity between bacterial populations within the cecum of mice fed the different diets. Each symbol represents a single mouse. The closer together two points are in the figure, the more similar the libraries are to one another. Libraries with similarities greater than $40 \%, 60 \%$ or $80 \%$ are encircled with blue, teal or red dashed lines, respectively. Selected vectors (lettered A-U), overlaid on the figure, were taken from a PCA plot made from all phylotype abundances in this study after identification by LEfSe analysis as having statistically significant differential abundances in mice fed different diets. B. 
LEfSe output highlighting differentially abundant phylotypes between Jax mice fed the two separate diets. Some of these phylotypes are included in A. as vectors. 

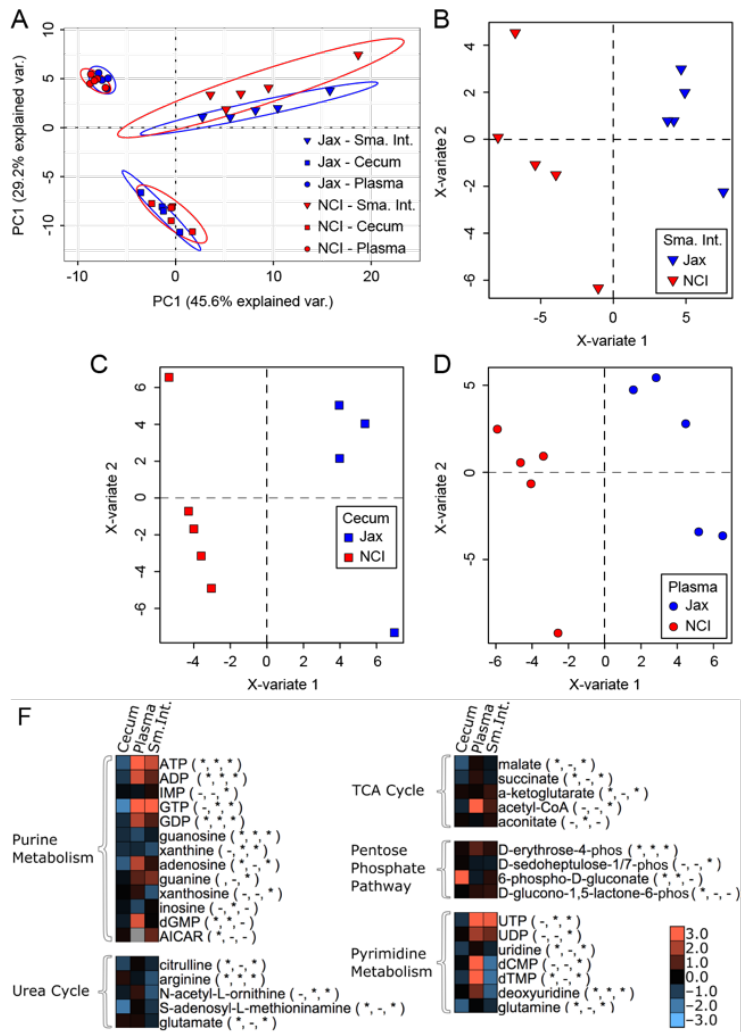

$\mathrm{E}$

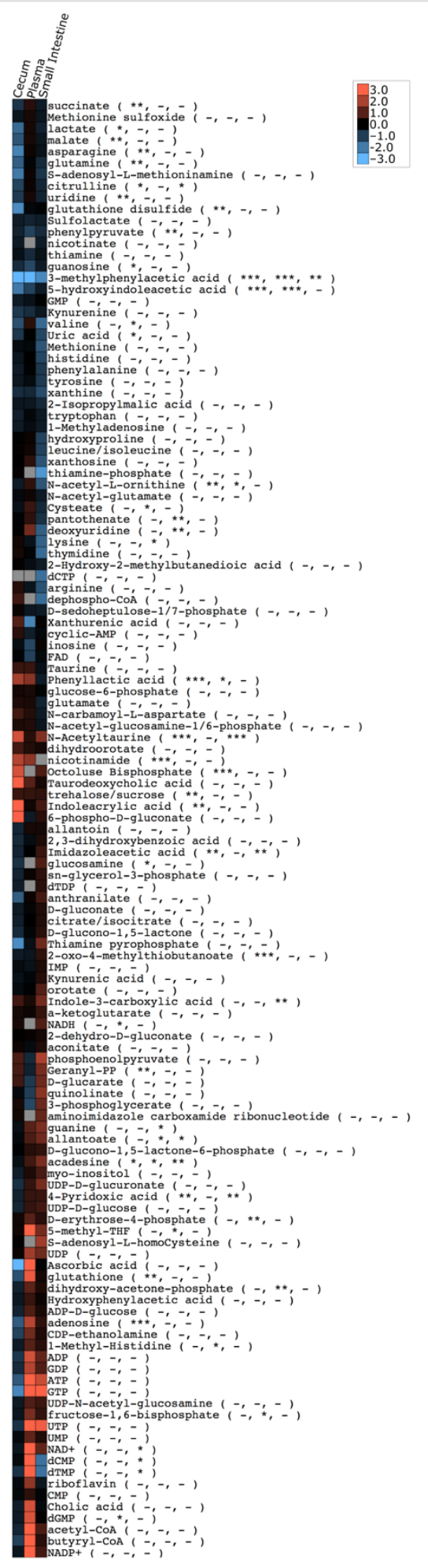

Figure 2-11. Changes in the gut microbial community structure correlate with changes in the gut and plasma metabolome in resistant and 
susceptible mice. A. Principal component analysis of metabolite profiles for all sample types. Ellipses size indicates $95 \%$ normal probability. Triangle=small intestine, square=cecum, circle=plasma. Each symbol represents an individual mouse. B-D. Partial least squares discriminant analysis within individual samples types: small intestine, cecum, and plasma, respectively. Separation between points indicates relative amount of variability. E. Color intensity indicates magnitude of the fold change with red being an increase and blue being a decrease in metabolite concentrations of Jax with respect to $\mathrm{NCl}$ mice. Asterisks in parentheses next to metabolite names indicate significance for the change for the corresponding row and column. ${ }^{* * *} p \leq 0.01,{ }^{* *} p \leq 0.05,{ }^{*} p \leq 0.10,(-)$ not significant. F. Metabolites grouped according to metabolic pathways that were major drivers in the differentiation of resistant and susceptible mice using PLSDA. Data are expressed as a ratio of Jax $/ \mathrm{NCl}$ with red shades representing metabolites higher in Jax mice and blue shades representing metabolites higher in $\mathrm{NCl}$ mice. $\left(^{*}\right)=$ Metabolite that was a driving factor in differentiating resistant and susceptible mice, respective to column order. $(-)=$ Metabolite that was not a driving factor in variation for the respective column. 


\begin{tabular}{|c|c|c|c|}
\hline \multicolumn{4}{|c|}{ Small Intestine } \\
\hline \multicolumn{2}{|c|}{ Jax Variability } & \multicolumn{2}{|c|}{ NCI Variability } \\
\hline Variate 1 & Variate 2 & Variate 1 & Variate 2 \\
\hline 3.84 & 5.23 & 6.90 & 10.86 \\
\hline \multicolumn{4}{|c|}{ Jax - NCI Variability } \\
\hline & Variate 1 & $\mathbf{Y}$ & \\
\hline Avg. Distance & 10.03 & 1.72 & \\
\hline$p$-value & 0.000269 & 0.411425 & \\
\hline \multicolumn{4}{|c|}{ Cecum } \\
\hline \multicolumn{2}{|c|}{ Jax Variability } & \multicolumn{2}{|c|}{$\mathrm{NCl}$ Variability } \\
\hline Variate 1 & Variate 2 & Variate 1 & Variate 2 \\
\hline 3.03 & 12.35 & 2.32 & 11.47 \\
\hline \multicolumn{4}{|c|}{ Jax - NCI Variability } \\
\hline & Variate 1 & Variate 2 & \\
\hline Avg. Distance & 9.12 & 1.76 & \\
\hline$p$ value & 0.000145 & 0.629464 & \\
\hline \multicolumn{4}{|c|}{ Plasma } \\
\hline \multicolumn{2}{|c|}{ Jax Variability } & \multicolumn{2}{|c|}{ NCI Variability } \\
\hline Variate 1 & Variate 2 & Variate 1 & Variate 2 \\
\hline 4.93 & 9.08 & 3.34 & 11.71 \\
\hline \multicolumn{4}{|c|}{ Jax - NCI Variability } \\
\hline & Variate 1 & Variate 2 & \\
\hline Avg. Distance & 8.23 & 2.36 & \\
\hline$p$ value & 0.000104 & 0.432392 & \\
\hline
\end{tabular}

Figure 2-12. Variability in each sample type for PLS-DA. For each sample type, the intra condition distance between the extreme values in each dimension are listed. Comparison is also shown for the distance between the average of the points for each condition in each dimension. Additionally, the $p$ values are shown for the inter conditional comparison. Results show a high level of significance between the Jax and $\mathrm{NCl}$ samples in the variate 1 dimension. 

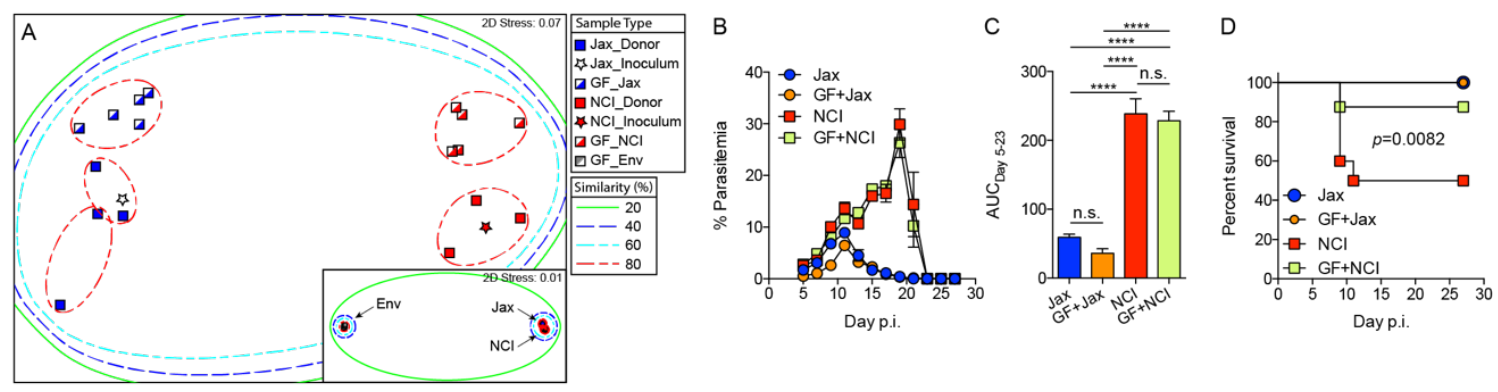

Figure 2-13. Gut microbiome shapes susceptibility to severe malaria. Germfree mice were colonized with cecal contents from Jax or NCI mice. A. Bacterial population analysis was performed using NMDS, as described in Fig. 2-11. B-D. Colonized germ-free mice and control Jax and $\mathrm{NCl}$ mice were infected with $P$. yoelii. B. Percent parasitemia following $P$. yoelii infection. C. AUC analysis. B-C. Data (mean \pm S.E.) from 4-5 mice/group are representative of two experiments. Data were analyzed by one-way ANOVA and Tukey's multiple comparison posttest. D. Survival of mice following infection. Data are cumulative results $(n=8-10$ mice/group) from two experiments. Survival curves were analyzed by Log-rank (Mantel-Cox) test. ${ }^{\star \star \star \star} p<0.0001$, n.s. $=$ not significant. 


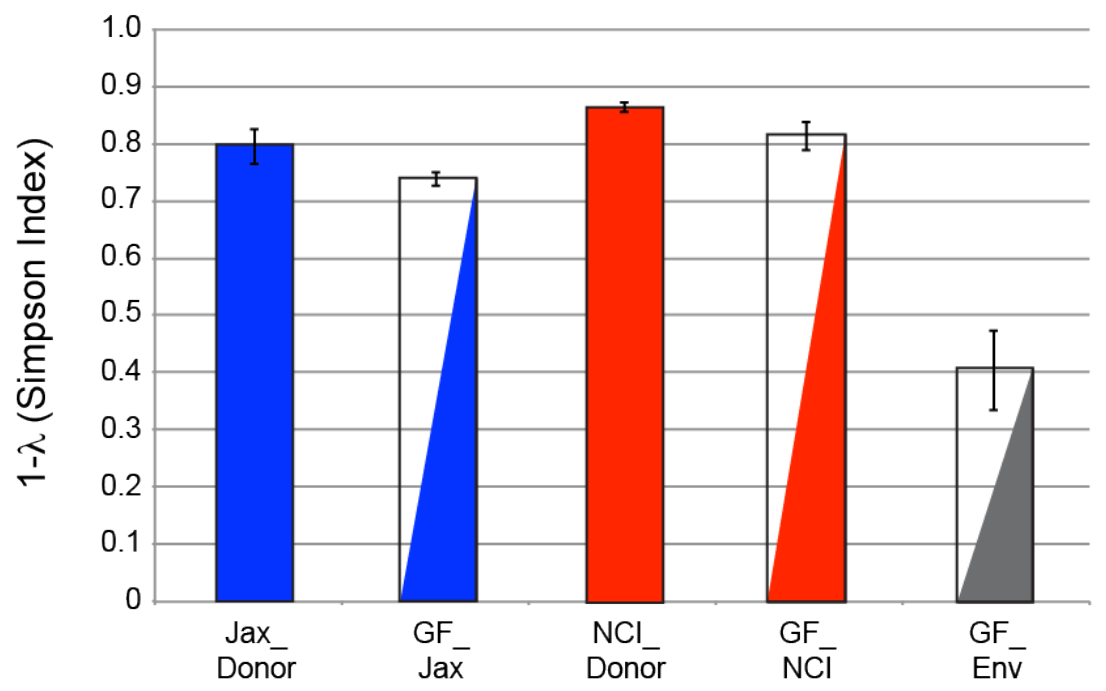

Figure 2-14. Germ-free mice colonized with cecal content have similar bacterial diversity as donor sample. Germ-free mice were colonized with cecal contents from Jax or $\mathrm{NCl}$ mice. The 1- $\lambda$ (Simpson Index) was calculated for 5 different mouse types (Jax-Donors $(n=4)$, GF-Jax recipients post-cecal transplant $(n=5), N C I$ Donors $(n=3)$, GF-NCI recipients post-cecal transplant $(n=5)$ and GF mice receiving no transplant $(n=5))$. Data are mean $\pm S D$. Communities with values closer to 1 are considered to be more diverse. 

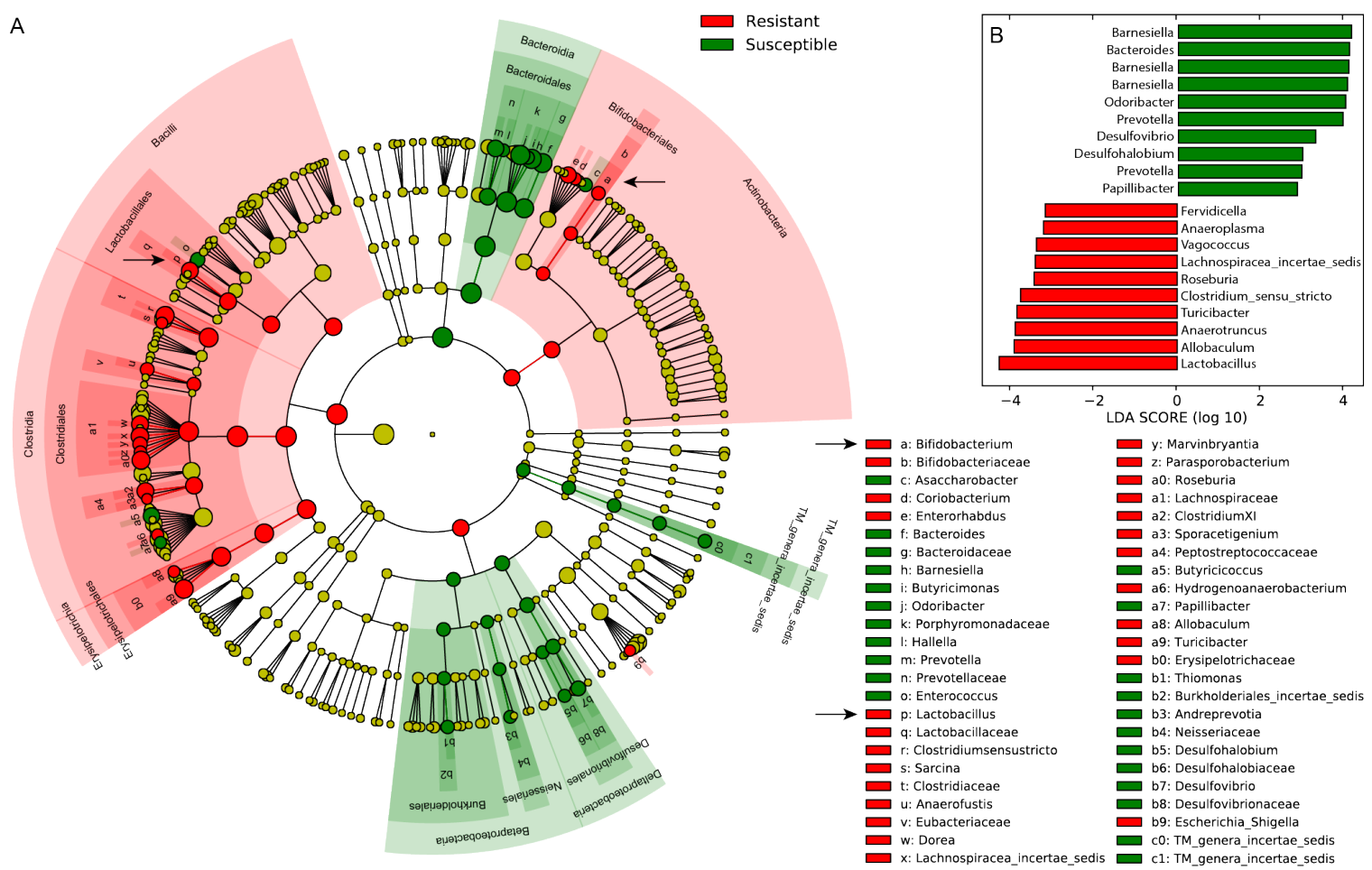

Figure 2-15. Lactobacillus and Bifidobacterium are among the bacteria that drive differences between the gut-associated bacterial communities of resistant and susceptible mice. A. LEfSe cladogram illustrating all 313 phylotypes in this study. Red identifies phylotype biomarkers for resistance to malaria and green identifies phylotype biomarkers for susceptibility to malaria. Circle size reflects the sequence abundance within the samples. B. Top ten phylotypes with largest effect sizes for susceptibility and resistance to malaria. 
$=$ Isolate
$\square=$ PCR Generated Clone

573 nucleotide alignment

$\longmapsto$

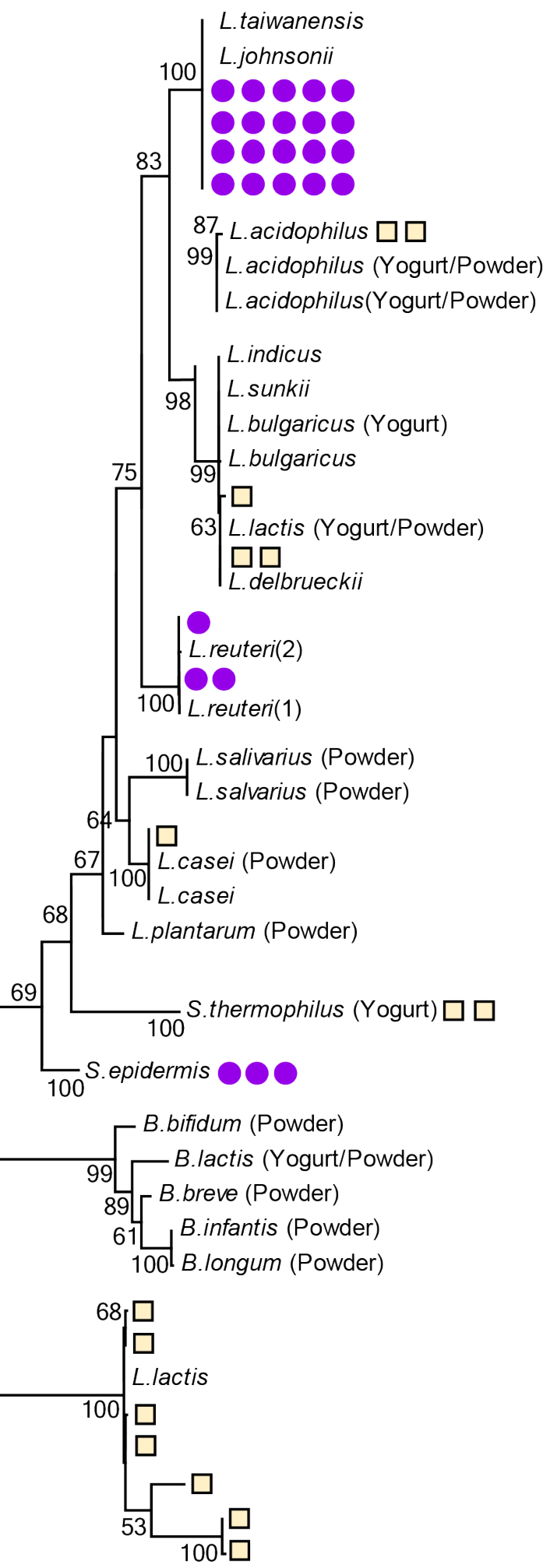

Figure 2-16. Phylogenetic tree of bacteria isolated from stool samples and

lab-cultured yogurt. A maximum likelihood tree was constructed from an 
alignment of $16 \mathrm{~S}$ rRNA gene sequences approximately 573 base pairs long.

Sequences used to construct the tree were obtained from bacterial isolates (cultured from Jackson and $\mathrm{NCI}$ mouse fecal pellets) and from PCR amplicons retrieved from DNA extracted from lab-cultured yogurt containing probiotic powder. Isolate sequences $=$ purple circles, clone sequences (yogurt or powder) $=\tan$ squares. Distance bar represents 0.1 substitutions per base.
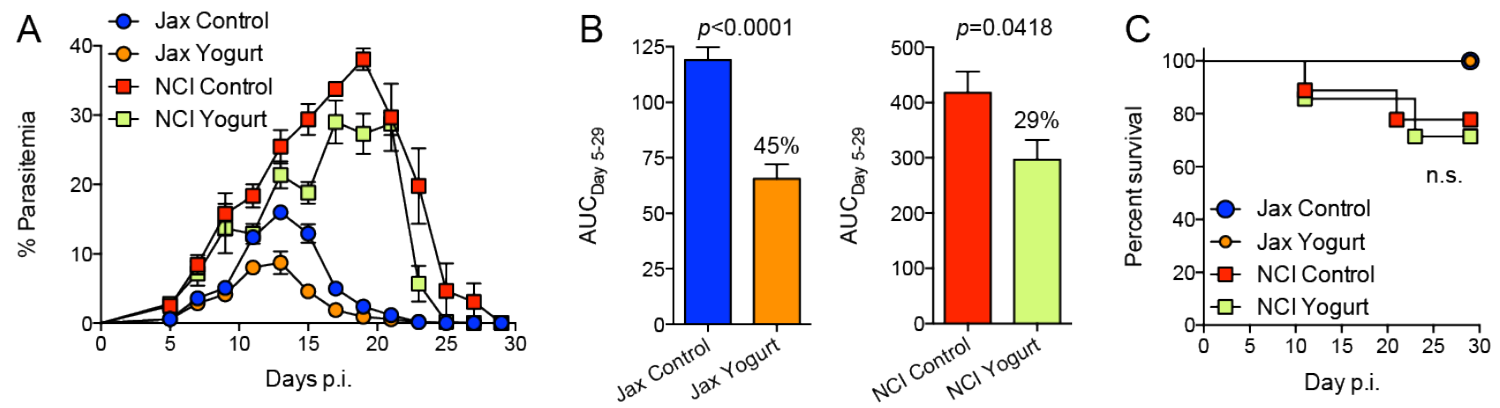

Figure 2-17. Treatment of gut microbiota intact mice with yogurt has a modest effect on parasite burden. Mice were treated 5-6 times/week with yogurt for 3 weeks prior to $P$. yoelii infection and 3 times per week following infection. A. Percent parasitemia following $P$. yoelii infection. B. AUC analysis. Data were analyzed by unpaired two-tailed t test. C. Survival of mice following infection. Survival curves were analyzed by Log-rank (Mantel-Cox) test. A-C. Data (mean \pm S.E.) from 7-9 mice/group are cumulative data from two experiments. 

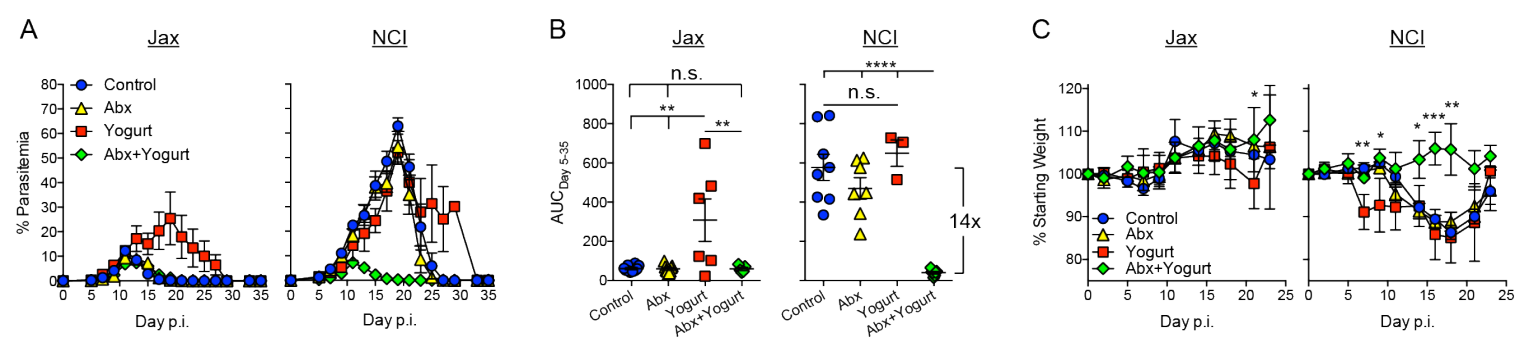

Figure 2-18. Susceptible mice treated with yogurt have decreased parasitemia and morbidity. Jax and $\mathrm{NCI}$ mice were left untreated (control), treated with antibiotics for three-weeks then left untreated for three additional weeks (Abx), left untreated for three-weeks followed by treatment with yogurt 5 times per week for three-weeks (Yogurt), or treated with antibiotics for threeweeks followed by treatment with yogurt 5 times per week for three-weeks (Abx+Yogurt). Mice were then infected with $P$. yoelii. Yogurt treated mice continued to receive yogurt 5 times per week following infection. A. Percent parasitemia following $P$. yoelii infection. B. AUC analysis. Data were analyzed by one-way ANOVA and Tukey's multiple comparison post-test. C. Percent weight loss following infection. Data were analyzed by one-way ANOVA. A-C. Data (mean \pm S.E.) are cumulative results ( $n=3-10$ mice/group) from two experiments. * $p<0.05,{ }^{* *} p<0.01,{ }^{* * *} p<0.001,{ }^{* * * *} p<0.0001$, n.s. $=$ not significant. 

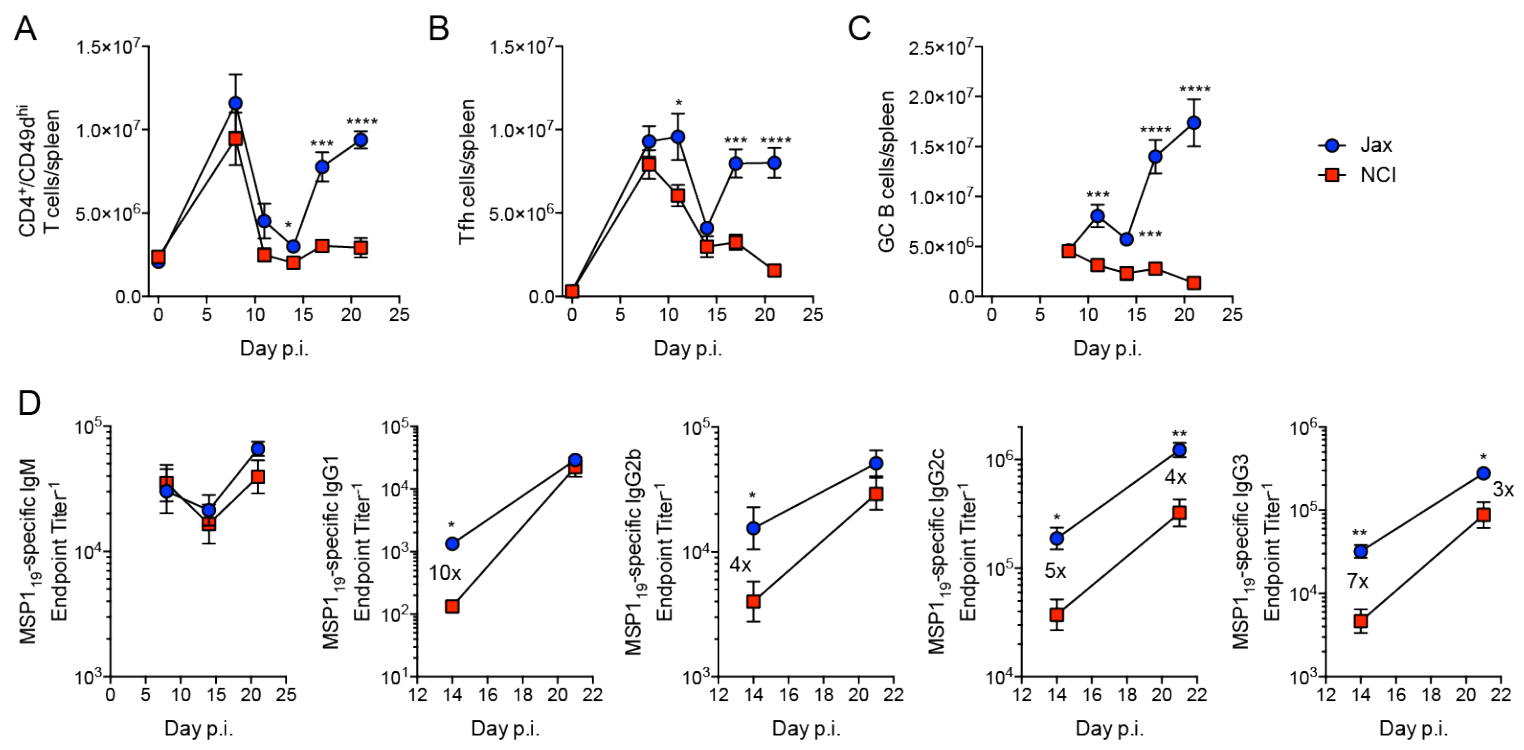

Figure 2-19. Resistant Jax mice have an elevated cellular and humoral immune response to Plasmodium. Jax and $\mathrm{NCl}$ mice were infected with $P$. yoelii. Total number of A. CD4+CD11ahiCD49dhi cells, B. T follicular helper cells, and C. germinal center (GC) B cells per spleen on the indicated day. Data (mean \pm S.E.) are cumulative results ( $n=5-10$ mice/data point) from three experiments. D. Serum MSP1 ${ }_{19}$-specific antibody endpoint titers. Data (mean \pm S.E.) are cumulative results ( $n=3-7$ mice/data point) from two experiments. Data were analyzed by unpaired two-tailed $t$ test. ${ }^{\star} p<0.05,{ }^{* \star} p<$ $0.01,{ }^{* * *} p<0.001,{ }^{* * *} p<0.0001$ 

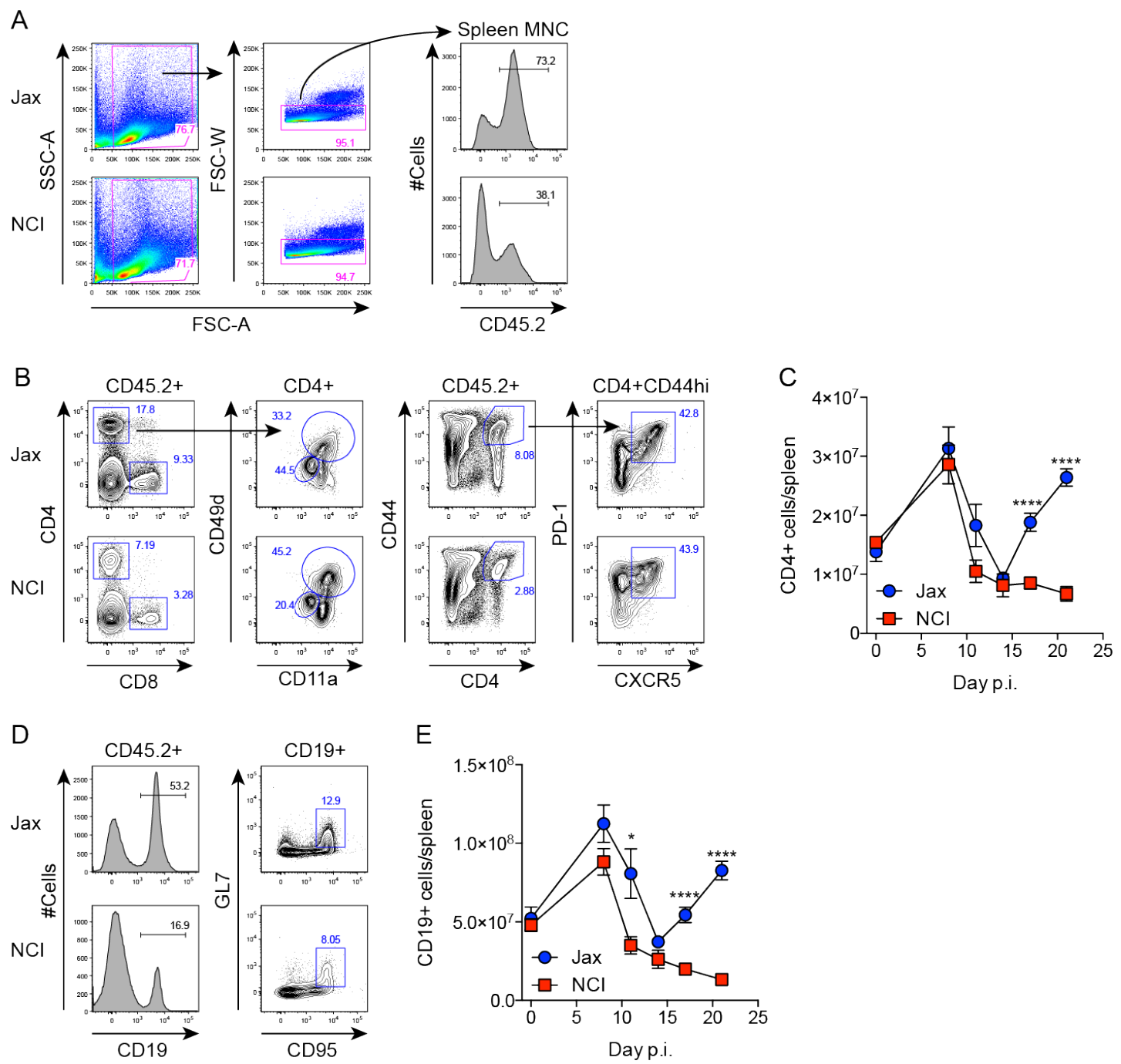

Figure 2-20. Gating strategy for $\mathrm{T}$ cell and $\mathrm{B}$ cell populations in Jax and $\mathrm{NCl}$ mice infected with $P$. yoelii. Jax and $\mathrm{NCl}$ mice were infected with $P$. yoelii. Representative contour plots and histograms are from day 21-post infection. A. Representative contour plots and histograms showing gating strategy of CD45.2+ cells. B. Representative contour plots showing gating strategy for T cell subsets: CD4+ T cells, $P$. yoelii-specific CD4+ T cells (CD4+CD49dhiCD11ahi), and T follicular helper (Tfh) cells (CD4+ CD44hiCXCR5+PD-1+). C. Total number of CD4+ cells per spleen. D. Representative contour plots and histograms showing 
gating strategy of $B$ cell subsets: CD19+ $B$ cells and GC B cells

(CD19+GL7+CD95+). E. Total number of CD19+ cells per spleen. Data

(mean $\pm S$.E.) are cumulative results $(n=5-10$ mice/data point) from three

experiments. Data were analyzed by unpaired two-tailed t test. ${ }^{*} p<0.05$, ${ }^{* * * *}$

$\mathrm{p}<0.0001$. 


\section{CHAPTER 3}

\section{SEVERITY OF PLASMODIUM INFECTION IN MICE DIFFERNTIALLY AFFECTS GUT-LIVER AXIS HOMEOSTASIS}

Malaria is a significant global health disease, with 216 million infections and 445,000 deaths occurring annually. With no effective vaccine and increasing anti-malarial drug resistance, it is imperative to gain a greater appreciation of the factors that influence the pathogenesis of this infectious disease. Recent reports have demonstrated dual interactions between gut microbiota and malaria. For example, in specific Plasmodium-mouse strain combinations, malaria has been shown to transiently modulate gut microbiota and cause intestinal shortening, indicating a disruption of gut homeostasis. However, these changes have not been characterized in the context of mild versus severe malaria. We show that severe Plasmodium infection in mice disrupts homeostasis along the gut-liver axis in multiple ways compared to mild infection. High parasite burden results in a larger influx of immune cells in the lamina propria and mice with high parasitemia display specific metabolomic profiles in the ceca and plasma during infection compared to mice with mild parasitemia. Liver damage was also more pronounced and longer lasting during severe infection, with concomitant changes 
in bile acids in the gut. Finally, severe Plasmodium infection changes the functional capacity of the microbiota, enhancing bacterial motility and amino acid metabolism in mice with high parasite burden compared to a mild infection. Taken together, Plasmodium infections have diverse effects on host gut homeostasis relative to the severity of infection. 


\section{Introduction}

Malaria infections, caused by Plasmodium, have long been of global clinical importance, with 216 million infections and approximately 445,000 deaths in $2016^{1}$. Several factors play a role in disease epidemiology, such as emerging resistance to frontline antimalarials ${ }^{124,125}$ and the lack of an effective vaccine. Further complicating efforts to eradicate this parasite is the dual life cycle wherein Plasmodium sexually reproduces and develops in its reservoir, the Anopheles mosquito, before being transmitted to the human host during a mosquito blood meal ${ }^{3,126}$. Within the human host the parasite undergoes development in the liver followed by asexual reproduction in red blood cells 3,126 . The liver stage is clinically silent, while the blood stage is associated with the clinical symptoms of malaria, including fever, anemia, and coma ${ }^{3,126}$.

The microbiota, which is the microbial consortia associated with the host, has been connected to host homeostasis and development. Bacteria are the most common inhabitants, while fungi and archaea make up smaller parts of the consortia. Indeed, recent calculations estimate there are approximately 3-4 x $10^{13}$ associated bacteria, corresponding to a bacteria:human cell ratio from around 1.3 to 2.3 depending on variables such as gender, age, and obesity ${ }^{127}$. The host microbiota has been examined in different sites such as skin ${ }^{128,129}$ and lung ${ }^{130-132,}$ but the gut microbiota has by far been the most studied. Intriguingly, the gut microbiota has also been shown to interact with the central nervous system in the "gut-brain axis", and is involved in processes like host development, circadian 
rhythm, and disease states such as major depression 81,133 . The gut microbiota has been shown to play a role in immunity, both locally in the intestine but also systemically in modulating host responses to diseases such as influenza and Klebsiella pneumonia ${ }^{71,73,134,135}$.

Likewise, it has previously been shown that Plasmodium infection and the severity of malaria can be modulated by the composition of gut microbiota. Antibody cross-reactivity with the gut commensal E. coli O86:B7 and the expressed malaria antigen Gala1-3Galß1-4GlcNAc-R (alpha-gal) leads to inhibition of sporozoite transmission through the $\operatorname{skin}^{74}$. We have previously observed that gut microbiota composition in mice can modulate the severity of $P$. yoelii 17XNL (Py) infection, and that susceptibility or resistance can be transferred to germ-free mice by transferring cecal contents from either susceptible or resistant mice ${ }^{136}$. Conversely, the Plasmodium infections also affect gut microbiota. Following Plasmodium berghei ANKA infection in C57BL/6 mice, which causes experimental cerebral malaria in C57BL/6 mice, gut microbiota became dysbiotic, or disrupted, as the infection progressed 38 . The authors concluded that the change in microbiota composition was due to $P$. berghei ANKA infection and not infection-associated inflammation, as the microbiota changes occurred before the intestines underwent inflammationmediated changes such as the intestine and villi shortening ${ }^{38}$. In contrast, there were little to no changes in gut bacteria observed in $P$. berghei ANKA infected BALB/c mice ${ }^{38}$. Finally, C57BL/6 mice infected with Plasmodium yoelii nigeriensis 
also exhibited changes in gut microbiota composition during peak infection, although these changes were transient with the composition returning to baseline following resolution of the infection ${ }^{37}$.

While it has been shown that gut microbiota affects and is affected by Plasmodium infection, these findings have not been explored in the context of mild vs. severe malaria. Using a mouse model of malaria, we show that severe malaria differentially disrupts gut homeostasis compared to a mild infection. Severe Py infection leads to more proinflammatory cell infiltration in the intestinal lamina propria, as well as differential metabolic changes during infection. Severe infection also leads to prolonged liver damage; surprisingly, mild Py infection led to longer-lasting changes in cecal bile acid abundances. Following infection there were shifts in the taxonomy of gut bacteria in both mild and severe Py infections, with the composition of the gut bacteria becoming more similar over the course of infection. Of note, these changes did not impact the severity of malaria in subsequent infections. Finally, severe infection drives a differential functional profile in the gut microbiota compared to mild infection. These results show that severe Py infection can differentially disrupt gut homeostasis in numerous ways.

\section{Results}

Intestinal Permeability Increases During Py Infection but is not a Function of Parasite Burden 
During Py infection, mice from Taconic Biosciences (Tac) and Charles River Laboratories (CR) show different parasitemia kinetics, leading to significantly different overall parasite burdens with CR mice exhibiting higher parasitemia than Tac mice (Fig. 3-1A and B). CR mice also display more weight loss, indicating greater morbidity during infection (Fig. 3-1C). These characteristics allow us to contrast a relatively mild Py infection in Tac mice to a severe Py infection in CR mice. We also looked at intestinal permeability during infection as one factor in gut homeostasis. While there were increases in intestinal permeability within the Tac and CR groups during infection, there were no differences between Tac and CR (Fig. 3-1D and E) indicating that increased intestinal permeability is not a function of overall parasite burden.

\section{The Lamina Propria Immune System Undergoes Differential Changes During Mild and Severe Py Infections}

The lamina propria (LP) houses a very diverse immune cell population responsible for maintaining tolerance to gut microbiota ${ }^{137-139}$. Additionally, it has been shown that a systemic infection like influenza can modulate the LP immune system ${ }^{71,73}$. With this in mind, we followed specific immune cell populations in both the small intestine (SI) LP and large intestine (LI) LP during Py infection. In general, CR mice had significantly more immune cells in the SI LP during infection than Tac mice (Fig. 3-2 and Fig. 3-9), particularly as the infection progressed and parasitemia peaked around days 14-21 post-infection (p.i.). More 
specifically, macrophages (CD45+SiglecF-CD11b+Ly6G-Ly6C-F4/80+) and CD8 T cells (CD45+CD8 $\left.{ }^{+}\right)$peaked at day 14 (Fig. 3-2B, I; Fig. 3-9), while monocytes (CD45+SiglecF-CD11b+Ly6G-Ly6C+) and neutrophils (CD45+SiglecFCD11b+Ly6G+) peaked at day 21 p.i. in CR mice (Fig. 3-2J, K; Fig. 3-9). The influx of monocytes and neutrophils indicates a more inflammatory LP environment for CR mice at the peak of Py infection, which could cause changes in gut microbiota. Tac mice similarly had an increase in CD8 T cells and macrophages at day 14 , along with a day 14 increase in neutrophils that correlate with peak parasitemia in Tac mice (Fig. 3-2B and I-J). Additionally, after Py clearance in the Tac SI: CD8 T cells (Fig. 3-2B), macrophages (Fig. 32I), and monocytes (Fig. 3-2J) all increased at day 60 p.i. In the LI LP (Fig. 310), there were fewer changes than in the SI LP: macrophages increased in Tac and CR LI at day 14 and day 60 p.i. (Fig. 3-10I) and IL17+ Th17 T cells (Fig. 310H) increased in both Tac and CR mice after Py clearance. While both Tac and CR had a significant increase in IL17+ gamma delta T cells (TCR $\gamma \delta$; CD45+TCR $\left.\gamma \delta^{+} \mathrm{IL}^{1+}\right)$ at days 7 and 14 p.i., Tac mice had more than CR at day 14 (Fig. 3-10F). Overall, distinct immune populations change during mild and severe malaria, with a potentially more inflammatory environment during severe malaria in CR mice.

Gut Microbiota Undergoes Significant Post-Infection Changes but does not Affect Susceptibility to Future Infections 
It has been shown previously that baseline gut microbiota composition in resistant and susceptible mice is sufficient to modulate the severity of infection ${ }^{136}$, but it has also been shown that the gut microbiota can experience Plasmodium-induced inflammation-related changes 37,38 . To initially determine how severity of infection impacts changes to bacterial community compositions over the course of infection, mice from Tac and CR were infected with Py and cecal contents were extracted for 16S rRNA (V6-V8) sequencing. While this was not a truly longitudinal analysis, it showed changes in both Tac and CR mice after infection, primarily after clearance of Py (Fig. 3-11). To confirm these results in a longitudinal analysis and increase the taxonomic assignment depth, Tac and CR mice were infected with Py and fecal pellets were collected at days $0,7,14,21,28,42$, and 56 p.i. for analysis of gut bacteria (Fig. 3-3A). Extracted DNA from fecal pellets was subjected to bacterial community analysis using $16 \mathrm{~S}$ rRNA gene sequencing based on a new technology that simultaneously utilizes all 9 hypervariable regions (manuscript describing this method is under peer review).

During Py infection, there are changes in the relative species abundance in both Tac and CR mice (Fig. 3-3B). For example, the Tac microbiota becomes more diverse as soon as 7 days p.i., with a significant increase in species diversity (alpha diversity) measured by observed OTUs (Fig. 3-3C). This increase is based on the appearance of different bacterial species such as Stomatobaculum longum that are not present at day 0 p.i. (Fig. 3-3B,C). 
Compared to the Tac microbiota, the CR microbiota is significantly more diverse before and during infection (Fig. 3-3C); however, the species diversity of the CR microbiota does not change from baseline (Fig. 3-3C). The beta diversity, or dissimilarity, of bacterial communities in both the Tac and CR mice increases significantly during infection compared to their respective baselines, with an earlier increase in Tac mice at day 7 (Fig. 3-3D). The increase in beta diversity illustrates the taxonomic changes that occur due to Py infection. Of note, BrayCurtis dissimilarity values range from 0 (identical similarity) to 1.0 (complete dissimilarity). Thus, while the increases in beta diversity are significant, they are relatively modest in both $\operatorname{Tac}$ (mean; day $0=0.19$ to day $56=0.40$ ) and $C R$ mice (mean; day $0=0.12$ to day $56=0.23$ ) with both Tac and CR communities, respectively, remaining relatively similar amongst themselves. These data demonstrate that Py infection alone, in contrast to the severity of infection, influences the observed changes in gut bacterial communities. Interestingly, the dissimilarity between the Tac and CR microbiota compositions decreases during infection (Fig. 3-3E), demonstrating a moderate convergence in the different microbiota compositions. Since different gut microbiota profiles have been shown to modulate susceptibility to Py infection ${ }^{136}$, these changes were investigated to determine how they impact susceptibility to future Py infections.

Mice infected with Py develop sterilizing immunity to Py after one infection, precluding the ability to directly reinfect these mice. Therefore, cecal contents were taken from Py-infected Tac and CR mice on days 56, 57, 60, and 61 p.i. 
and gavaged into germ-free (GF) mice (Fig. 3-3A). The recipient mice, along with non-gavaged control Tac and CR mice, were infected with Py seven days after the last cecal-content transplant and parasitemia was tracked.

To confirm the GF mice had been colonized properly, fecal samples from recipient mice were collected on the day of Py infection (Fig. 3-3A) for bacterial community analysis. The species diversity of both the GF+PyTac and GF+PyCR samples was similar to the input diversity, which means the GF mice were successfully colonized and their gut bacteria populations mirror the donor mice that had previously been infected with Py (Fig. 3-3F). More specifically, the Tac control samples had significantly lower species diversity than the input contents (Fig. 3-3F), confirming the observed changes during Py infection (Fig. 3-3C). Similarly, the Tac input and GF+PyTac samples are significantly dissimilar to the Tac controls but not each other (Fig. 3-3G). In the CR samples, the CR input samples had a slightly higher species diversity than the CR control (Fig. 3-3F), but this did not result in a bacteria community that was overall dissimilar (Fig. 33G). Following infection, the GF+PyTac and GF+PyCR contents phenocopied parasitemia in the respective Tac and CR control mice with regards to the infection kinetics (Fig. 3-3H) and overall parasite burden (Fig. 3-3I). These data suggest that Py-induced changes in gut bacterial communities do not change resistance or susceptibility to future Py infections.

\section{Cecal and Plasma Metabolite Profiles Vary Between Tac and CR Mice}


One mechanism by which gut microbiota can influence host homeostasis is through the production of metabolites. Different metabolites can have various effects on the host: nucleotides in the gut can reduce inflammation, while tryptophan metabolites can activate the aryl hydrocarbon receptor (AhR) and lead to an anti-inflammatory and xenobiotic clearance response ${ }^{140-142}$. To this end, SI and cecal contents as well as plasma were extracted from mice at days $0,7,14,21,28$, and 60 p.i. and characterized by mass spectrometry.

In the SI, no distinct clustering between Tac and CR samples is observed, with the exception of two noted outliers, one Tac sample at day 7 p.i. and one CR sample at day 0 p.i. (Fig. 3-4A-B). In contrast, the cecal PCA plot shows distinct differences between Tac mice and CR mice (Fig. 3-4C). In the CR mice, a similar metabolite profile is seen at days 0,7 , and 14 p.i.; however, at day 21 p.i., which correlates to the peak of severe infection, the metabolites in the cecum become much more abundant (Fig. 3-4D). This enrichment decreases by day 28 p.i. to below-baseline levels for metabolites in the bottom-half of the heatmap and remain low even one month after clearance of the infection. In contrast to CR mice, cecal metabolites remain largely stable in Tac mice over the course of infection, with only a few metabolites decreasing at days 28 and 60 p.i. (Fig. 34D). Tac and CR plasma metabolite profiles follow similar kinetics during infection. The most pronounced changes during infection are the similar shifts in metabolites at the peak of infection: day 14 p.i. in Tac mice and days 14 and 21 p.i. in CR mice (Fig. 3-4E). The clustering of these samples explains a robust 
$70 \%$ of the variation. The metabolite profiles seen in naïve mice become inverted, with abundant metabolites at day 0 p.i. becoming depleted, while less abundant metabolites becoming enriched at the peaks of infection in Tac and CR mice (Fig. 3-4F). In addition, after Py clearance, the metabolite profiles in both Tac and CR mice largely return back to pre-infection levels by day 60 p.i. (Fig. 34F).

Overall, Tac and CR mice have different metabolite profiles before and during infection, and the changes in cecal metabolite concentrations during infection correlates with the severity of infection. Meanwhile, the plasma metabolite dynamics appear to depend on the kinetics of infection more so than the severity of infection.

\section{Py Infection Causes Differential Liver Damage in Mice During Py Infection}

Differential cecal metabolites, noted gross anatomical changes in the liver of Py infected mice (data not shown), and the observation that Plasmodium infections cause fibrotic lesions in the liver ${ }^{143}$ led us to investigate a potential role of the liver in Py-induced changes in gut homeostasis. The gut-liver axis has been well-established ${ }^{144-147}$, and can influence metabolite profiles and microbial community structures through different mechanisms. Livers were extracted from mice at days $0,7,14,21$, and 28 days post-Py infection and stained with hematoxylin and eosin (H\&E). Before infection, normal liver structure is observed in Tac and CR mice (Fig. 3-5). After Py infection, immune cells begin to infiltrate 
the liver and disruption of the liver architecture can be seen. In CR mice, the infiltrating cells remain in close proximity to the vasculature, while in Tac livers the infiltrating cells appear to invade further into the liver tissue (Fig. 3-5). At day 14, which is around peak parasitemia for Tac mice, immune cell infiltration increases and modest hemozoin deposition is seen (Fig. 3-5). In CR mice at day 14 p.i., the immune cell infiltration has progressed deeper into the tissue, and more extensive hemozoin deposition is seen compared to the Tac livers; the liver architecture also becomes further disrupted (Fig. 3-5). By day 21 p.i., Tac mice have cleared the infection; however, low levels of hemozoin and infiltrating immune cells are still present (Fig. 3-5). At day 28, the Tac liver architecture has returned to its pre-infection state and the infiltrating immune cells have left, but small amounts of hemozoin can still be seen within the tissue (Fig. 3-5). In comparison, day 21 p.i. is peak parasitemia for the CR mice, which display a more severe infection. Consistently, the liver architecture is highly disrupted, with infiltrating immune cells and hemozoin scattered throughout the tissue (Fig. 3-5). Liver architecture is partially restored by day 28 with only few infiltrating immune cells around the vasculature (Fig. 3-5). Compared to Tac livers at day 21 and day 28 p.i., there is notably more hemozoin in CR livers. Collectively, the severity of blood stage infection leads to much more pronounced and prolonged damage to the liver. 


\section{Tac and CR Mice Have Different Bile Acid Profiles Before and During Py Infection}

One mechanism by which the liver can influence gut microbial communities is through bile acid production. Bile acids are detergent-like molecules that can be metabolized by gut bacteria, disrupt bacterial cell membranes, and act as signaling molecules to the intestinal epithelial cells ${ }^{148-151}$. We hypothesized that bile acid production would be altered as a function of the severity of infection. To test this hypothesis, bile acids were analyzed at days 0 , $7,14,21,28$, and 60 p.i. in the SI, cecum, and plasma of Tac and CR mice. In the SI, the most notable pattern in bile acid expression is that the Tac mice have a significant decrease in almost half of the detected bile acids at day 14 compared to pre-infection bile acid levels (Fig. 3-6A, Fig. 3-12). In terms of individual bile acids, conjugated bile acids glycochenodeoxycholic acid (GCDCA), taurochenodeoxycholic acid (TCDCA), and taurodeoxycholic acid (TDCA) in Tac and CR show significant changes at several time points (Fig. 312).

In the cecum, CR mice tend to have higher relative bile acid concentration before and during infection compared to Tac mice (Fig. 3-6B, Fig. 3-13). However, both Tac and CR mice exhibit a significant decrease in specific bile acids during infection, with the largest changes occurring in the taurineconjugated bile acids. In particular, taurine-conjugated bile acids become depleted at day 14 in Tac mice with a mild recovery before a long-term depletion 
up to day 60 p.i. (Fig. 3-6B, Fig. 3-13). CR mice have a similar depletion at day 14 that extends to day 21 p.i., consistent with the more severe infection in CR mice, but these bile acid levels return to baseline levels by clearance of the parasite at day 28 p.i. (Fig. 3-6B, Fig. 3-13). Additionally, several bile acids are significantly higher in CR mice over the course of the infection compared to Tac, such as glycocholate (GCA) and omega-muricholate (oMCA) (Fig. 3-6B, Fig. 313).

Plasma bile acids were low in abundance and were largely unchanged over the course of infection. The two noted exceptions were in Tac mice: glycineconjugated bile acids GCDCA and glycodeoxycholate (GDCA) decrease at days 7 and 14 p.i. and day 7 p.i., respectively (Fig. 3-6C, Fig. 3-14); alpha-muricholate (aMCA) significantly decreases at days 7 and 14 p.i. while beta-muricholate (bMCA) is significantly more abundant at those same time points (Fig. 3-14).

Like the metabolomics data (Fig. 3-4), the cecal bile acid profiles in Tac and $\mathrm{CR}$ are different at baseline and change differentially due to mild or severe infection. In this case, however, the milder Py infection induces long-lasting depletion of bile acids.

\section{Predicted Functional Changes in the Microbiota Align with Combined Datasets}

We have observed many changes within the host and the microbiota during infection. To investigate how the observed taxonomic and metabolite 
profiles interact, PICRUSt was utilized to predict the functional capacity ${ }^{152}$ of cecal bacteria in Tac and CR mice based on the sequencing data in Figure 3-11. Once in PICRUSt, KEGG ortholog (KO; a characterized gene or protein within a network) abundances were assigned to each sample; KOs were then categorized according to the KO reference hierarchy and analyzed ${ }^{153-155}$. When naïve Tac and $\mathrm{CR}$ mouse $\mathrm{KO}$ abundances are compared, the proportional abundances of each category are similar, but in spite of the lower species diversity (Fig. 3-11B) the naïve Tac microbiota have approximately $40 \%$ more KOs than the CR microbiota (Fig. 3-7A,B). "Metabolism" was the largest category, containing almost $47 \%$ of total KOs during infection. Tac microbiota possess significantly more KOs than CR mice at days 0 and 7 p.i., while from day 14 p.i. onward the Tac and CR microbiota possess similar KO abundances (Fig. 3-7C). The observed change in the functional capacity in the CR microbiota by day 14 p.i. precedes the large taxonomic changes and metabolomics changes observed at day 60 p.i. and day 21 p.i., respectively. Each level 1 KEGG category follows this same pattern except for one, "Cellular Processes"; Tac and CR microbiota are significantly different at day 7 p.i., but not at day 0 , indicating that Py infection differentially affects the Tac and CR microbiota during early infection (Fig. 3-7D). Similarly, the CR microbiota undergoes significant functional changes in Cellular Processes at day 21 p.i. compared to the day 0 p.i. CR microbiota (Fig. 3-7D). The only level 2 subcategory under Cellular Processes that also changes significantly from day 0 to day 21 p.i. is "Cell Motility", which includes flagellar 
assembly and bacterial chemotaxis (Fig. 3-7E). Finally, the Cellular Processes KOs at day 28 and day 60 p.i. correlate with the taxonomic changes: the Tac microbiota changes significantly at day 28 p.i., while the $\mathrm{CR}$ microbiota shows a delayed change at day 60 p.i., effectively "catching up" to the Tac microbial community structure. When comparing the PICRUSt data to the taxonomic data in Fig. S3, the bloom in the S24-7 bacterial family (Fig. 3-11A) correlates to a loss in the Cellular Processes functional capacity, and more specifically a loss in the microbiota's capacity for cell motility (Fig. 3-7D,E). Taken together, naïve Tac and CR microbiota each have distinctly different functional profiles; after Py infection, the functional capacity of these two bacterial communities become indistinguishable, even after the parasite is cleared. In the case of some KO categories like Cellular Processes and Cell Motility, the KO abundances correlate closely with the taxonomic changes seen in Tac and CR microbiota during convalescence.

The metabolomics data can be used to validate the predicted functionality of the cecal microbiotas. Tac mice are predicted to have a significantly higher functional capacity for "Amino Acid metabolism" (Fig. 3-7F) compared to CR mice. When compared to the observed metabolite abundances, the top enriched pathways at day 0 p.i. are related to metabolism of various amino acids, and this enrichment is associated with the Tac phenotype (Fig. 3-7G). In contrast, at day 21 p.i., the enriched pathways have changed and the majority are now associated with the CR phenotype (Fig. 3-7H); this shift correlates to the large 
increase in metabolite abundances in the CR cecum at day 21 p.i. (Fig. 3-4D).

Overall, the functional predictions in the ceca of Tac and CR mice fit well with the taxonomic and metabolomic data presented.

\section{Discussion}

In this study, we have shown that gut homeostasis is differentially disrupted by severe malaria compared to mild malaria. We examined several factors of gut homeostasis, including intestinal permeability, the intestinal immune system, gut microbiota and metabolites, and the gut-liver axis.

Altogether, severe malaria differentially influences gut homeostasis with distinct actions (Fig. 3-8). During infection, the LP in CR mice with severe malaria has a larger influx of CD8 T cells, monocytes, neutrophils, and TCR $\gamma \delta$ cells, all of which can produce proinflammatory cytokines. The microbiota of both Tac and CR mice differentially change during infection, with larger changes occurring earlier in Tac mice. The taxonomic changes in Tac and CR mice lead to enrichment of different bacterial species, highlighting the differential modulation of microbiota by Py infection. Initially, CR microbiota have a sparse predicted functional profile compared to Tac microbiota; during infection, the CR and Tac predicted functional capacity becomes more similar, reflecting the taxonomic similarity at the family level post-Py clearance. The cecal metabolite profiles in CR mice reflect the parasite burden, with the peak of infection mirroring a large increase in metabolite abundances, while Tac mice do not show a similar increase. CR mice 
also show major liver damage during infection with more extensive damage and hemozoin deposition compared to asymptomatic Tac mice. Finally, cecal bile acid profiles in Tac mice show a prolonged depletion during infection compared to $\mathrm{CR}$ mice, with $\mathrm{CR}$ bile acid abundances generally returning to baseline levels by days 28 and 60 p.i. Taken together, these data show patterns of gut homeostasis disruption during severe malaria.

While previous studies have shown changes to gut microbiota during Plasmodium infection as well, our data is unique in that it shows that there are long-term changes that occur during infection that persist after the infection is cleared. Mooney et al. showed that during Plasmodium yoelii nigeriensis infection, there were shifts in murine gut microbiota, but these returned to baseline within 30 days p.i. and were taxonomically restricted primarily to the phylum level with only a few noted changes at the genus leveli3. Taniguchi et al. also showed shifts in the gut microbiota of C57BL/6 mice infected with $P$. berghei ANKA, which models cerebral malaria, but not BALB/c mice, which do not model cerebral malaria ${ }^{38}$. Of note, all the $P$. berghei ANKA infected C57BL/6 mice succumbed to cerebral malaria, so it is unknown if these changes affect parasite burden, immunity to malaria, or would remain after clearance of infection. We have extended these analyses to show that the gut microbiota undergoes longterm, persistent changes down to the species level due to Py infection and that these changes do not affect susceptibility to future Py infections. 
We have also looked more closely at the LP immune response during Py infection. Previously, it had been observed that during $P$. yoelii nigeriensis infection, mononuclear cells infiltrate the LP up to 10 days p.i., with a large portion of these identified as inflammatory monocytes (Ly6C+ Ly6G-) ${ }^{38}$. Using Py 17XNL instead of $P$. yoelii nigeriensis, we have also found that large numbers of monocytes infiltrate into the LP of mice with high parasitemia 21 days p.i. before returning to baseline, while mice with low parasitemia show a significant increase 60 days p.i. In addition, we have observed a significant increase in macrophage infiltration at 7 days p.i. and 60 days p.i. We have corroborated the previously observed early mononuclear cell infiltration and extended the analysis further p.i. and to more cell types.

A potential mechanism for how severe malaria differentially impacts gut homeostasis may be due to parasite sequestration in the gut vasculature. In severe malarial anemia in humans, for instance, the bulk of parasite sequestration occurs in the vasculature of the small and large intestines; patients with cerebral malaria also show a large amount of parasite sequestration in the gut along with the brain ${ }^{156}$. Sequestration in the vasculature could lead to inflammation which in turn would damage the gut tissue, disrupting tissue homeostasis.

One promising avenue for further investigation within the malaria-gut microbiota axis involves bile acid metabolism in the gut. In the intestine, bile acids aid in digestion but can also behave as signaling molecules or 
bacteriostatic agents. As detergent-like molecules, bile acids can weaken or destroy bacterial cell membranes, keeping bacterial growth in check ${ }^{151,157 . ~ I n ~}$ both Tac and CR mice, the initial liver damage and depletion of bile acids correlates with the increase in gut bacterial diversity during and after infection, pointing to the potential role of bile acids in shaping the gut microbiota before and during Py infection.

Signaling by bile acids in the intestine can occur through the $G$ proteincoupled receptor TGR5. CDCA and lithocholic acid (LCA)/DCA are the strongest ligands, with other bile acids binding with lesser affinity; conjugation with taurine or glycine makes binding and activation more effective ${ }^{148-150}$. TGR5 is found in the intestine as well as in extra-intestinal tissues such as lung, spleen, and bone marrow ${ }^{158}$. Since plasma levels of bile acids did not vary between Tac and CR mice during infection, it is unlikely that activation of TGR5 in extra-intestinal tissue is involved. However, TGR5 activation on intraepithelial lymphocytes leads to the expression of GLP-1 (glucagon-like peptide 1) which in turn inhibits proinflammatory cytokine production ${ }^{159}$. As Tac mice have a greater abundance of taurine-conjugated bile acids than CR mice initially, TGR5 signaling may be play a protective anti-inflammatory role early in Py infection.

While there is still much work to be done, these results identify the complex network of interactions that influence gut homeostasis during Py infection in mice and provide an extensive characterization of how different factors in gut homeostasis respond during mild versus severe Py infection. Many 
of the perturbations in gut homeostasis were associated with a more severe infection. Given that many parasitized red blood cells sequester in intestinal villi in humans, it is paramount that future work begin assessing the effect of Plasmodium infections on human gut homeostasis.

\section{Materials and Methods:}

\section{Animals and Housing:}

Female C57BL/6 mice 6-8 weeks old were purchased from Taconic Biosciences (Hudson, NY) and Charles River Laboratories (Wilmington, MD). Germ-free (GF) mice were purchased from Taconic Biosciences. All mice were housed in a specific pathogen-free facility and acclimatized for a minimum of 7 days before starting experiments. Animals were fed the NIH-31 diet (Modified Open Formula Mouse/Rat Irradiated Diet; Harlan 7913; Envigo, Indianapolis, IN) and provided autoclaved, non-acidified municipal water ad libitum. The mice were kept on a 12-hour light/dark cycle from $6 \mathrm{AM}$ to 6PM and 6PM to 6AM, respectively. All animal experiments were carried out at the University of Louisville in compliance with local and national regulations of laboratory animal welfare. Additionally, all animal care and use procedures were approved by the University of Louisville Institutional Animal Care and Use Committee.

\section{Plasmodium Infection and Evaluation of Parasitemia:}


Mice were infected with Plasmodium yoelii 17XNL by intravenous injection of $1 \times 10^{5}$ infected red blood cells (RBCs) in $200 \mathrm{uL}$ of saline prepared from frozen stock. Parasitemia (i.e. percentage of total infected RBCs) was evaluated by flow cytometry between days 5-30 post-infection (p.i.) via blood taken from the tails of infected mice. Approximately $5 \mu \mathrm{L}$ of whole blood was diluted in $100 \mu \mathrm{L}$ of PBS followed by fixation in $0.00625 \%$ glutaraldehyde. The samples were then stained: CD45.2-APC (clone 104; Biolegend, San Diego, CA), Ter119-APC/Cy7 (clone TER-119; Biolegend, San Diego, CA), dihydroethidium (MilliporeSigma, St. Louis, MO), and Hoechst 33342 (MilliporeSigma, St. Louis, MO). After staining, samples were resuspended in flow cytometry buffer and analyzed; RBCs were gated by Ter119+CD45.2- followed by gating the infected subpopulation on dihydroethidium+Hoechst $33342+$ to find the percentage of infected RBCs.

\section{GF Cecal Content Transplant:}

Tac and CR cecal donor mice were infected with Py; at day 56 p.i., GF mice were received. GF mice were colonized immediately upon arrival with cecal contents from either the infected Tac or CR donor mice. GF mice were gavaged daily with cecal contents for a total of 4 treatments and then rested for 1 week before Py infection. Fecal pellets were collected after gavage to ensure colonization recapitulation of donor microbiota.

\section{Liver Histology:}


Liver samples were collected from mice at days $0,7,14,21$, and 28 p.i. Livers were extracted from mice, trimmed of connective tissue, and placed into Tissue-Tek Uni-Cassettes (Sakura Finetek, Torrance, CA) in 10\% neutral buffered formalin (MilliporeSigma, St. Louis, MO) for fixation. Livers were then processed using a graded ethanol series and embedded in paraffin. The paraffin sections were cut into $5 \mu \mathrm{M}$-thick slices using a microtome and stained with hematoxylin and eosin (H\&E). All stained sections were examined by light microscopy using an Olympus BX41 microscope. Representative images are shown (magnification - 20X).

\section{Intestinal Permeability Assay:}

Intestinal permeability was measured using 4kD FITC-dextran (MilliporeSigma, St. Louis, MO). Mice were fasted for 4 hours followed by oral gavage of $\sim 42 \mathrm{mg}$ FITC-dextran/100mg of body weight in $200 \mu \mathrm{L}$ of PBS, or $8.4 \mathrm{mg} / 200 \mu \mathrm{L} /$ mouse. Three hours post-gavage, serum was collected, diluted 1:1 in PBS to reach $100 \mu \mathrm{L}$ final volume, and read on a spectrophotometric plate reader for fluorescence intensity (Excitation at $485 \mathrm{~nm}$ and emission at $528 \mathrm{~nm}$ ).

\section{Lamina Propria Immune Cell Analysis:}

Lamina propria (LP) immune cells were isolated at days $0,7,14,21,28$, and 60 p.i. using the mouse Lamina Propria Dissociation Kit (Miltenyi Biotec, Auburn, $\mathrm{CA}$ ) according to the manufacturer's instructions. Briefly, small and large 
intestines were extracted from mice and cut open longitudinally and laterally into approximately $0.5 \mathrm{~cm}$ long pieces. The samples were then incubated and washed to dissociate the epithelial layer. The resulting samples were then run on a gentleMACS dissociator (Miltenyi Biotec, Auburn, CA) and filtered to obtain a single-cell suspension. Samples were split and either only surface stained or surface stained and intracellularly stained with fluorescence-conjugated antibodies. Antibodies were resuspended in FACS buffer (1x PBS, $0.02 \%$ sodium azide, and $1 \%$ FCS) for surface staining for 15 minutes at $4^{\circ} \mathrm{C}$ followed by fixation with Fixation Buffer (Biolegend, San Diego, CA), while intracellular staining was carried out with the eBioscience Foxp3/Transcription Factor Staining Buffer Set (ThermoFisher, Waltham, MA) according to the manufacturer's instructions. Samples were collected on a BD LSRFortessa (BD Biosciences, San Jose, CA) and analyzed using FlowJo software for Mac, version 10.4.2 (FlowJo, Ashland, OR).

\begin{tabular}{|l|l|l|}
\hline Marker-Fluorescence & Clone & Vendor \\
\hline CD4-APC-Cy7 & RM4-5 & Biolegend \\
\hline CD8-FITC & $53-6.7$ & Biolegend \\
\hline CD45-AF700 & 104 & Biolegend \\
\hline TCR $\gamma$-BV421 & GL3 & Biolegend \\
\hline CD19-PerCP-Cy5.5 & $6 D 5$ & Biolegend \\
\hline Foxp3-PE & $150 D / E 4$ & Invitrogen \\
\hline
\end{tabular}




\begin{tabular}{|l|l|l|}
\hline CD49b-APC & DX5 & Biolegend \\
\hline ROR $\gamma$-BV650 & Q31-378 & BD Biosciences \\
\hline IL17A-PE-Cy7 & TC11-18H10.1 & Biolegend \\
\hline CD3-FITC & $145-2 C 11$ & Biolegend \\
\hline CD11c-BV650 & N418 & Biolegend \\
\hline CD11b -PE-Cy7 & M1/70 & Biolegend \\
\hline Siglec F-PE & E50-2440 & BD Biosciences \\
\hline Ly6C-PerCP-Cy5.5 & AL-21 & BD Biosciences \\
\hline Ly6G-APC & 1 A8 & Biolegend \\
\hline F4/80-BV421 & BM8 & Biolegend \\
\hline CD16/32 (Fc Block) & $2.4 G 2$ & \\
\hline
\end{tabular}

\section{Metabolite Screening and Bile Acid Analysis:}

Ceca, small intestine, and plasma samples were collected from Tac and CR mice at days $0,7,14,21,28$, and 60 p.i. The ceca and small intestines were flushed with extraction buffer (a mix of 40:40:20 HPLC grade methanol, acetonitrile, and water with $0.1 \%$ formic acid overall), flash frozen in liquid nitrogen, and stored at $-80^{\circ} \mathrm{C}$. Samples were shipped overnight on dry ice to our collaborators for an untargeted metabolomics screen and targeted bile acid analysis. Sample preparation was the same for both mass spectrometric analyses. Shipped samples were extracted, dried, resuspended and immediately placed in a $4^{\circ} \mathrm{C}$ mass spectrometer autosampler according to a method 
previously described [19]. $10 \mu \mathrm{L}$ of sample was injected into the Ultra Performance Liquid Chromatography-High Resolution Mass Spectrometer (UPLC-HRMS), a Dionex Ultimate 3000 coupled to an Exactive Plus orbitrap mass spectrometer (Thermo Scientific, Walham, MA, USA).

The untargeted metabolomic screen achieved separations using a Synergy Hydro-RP column (100 mm x 2 mm, $2.5 \mu \mathrm{m}$ particle size, Phenomenex, Torrance, CA) at a flow rate of $200 \mu \mathrm{L} / \mathrm{min}$. The mobile phase consisted of $97: 3$ HPLC grade water:methanol, $11 \mathrm{mM}$ tributylamine, and $15 \mathrm{mM}$ acetic acid labeled as solvent A, as well as HPLC grade methanol, labeled as solvent B. The mobile phase gradient was programmed accordingly: From 0 to $5 \mathrm{~min}, 0 \% \mathrm{~B}$; from 5 to $13 \mathrm{~min}, 20 \% \mathrm{~B}$; from 13 to $15.5 \mathrm{~min}, 55 \% \mathrm{~B}$, from 15.5 to $19 \mathrm{~min}, 95 \%$ $\mathrm{B}$; and from 19 to $25 \mathrm{~min}, 0 \% \mathrm{~B}$, Eluent from the column was introduced into the mass spectrometer, an Exactive Plus orbitrap (Thermo Scientific, Waltham, MA, USA) via an electrospray ionization (ESI) source set to negative mode. Instrument settings include: spray voltage of $3 \mathrm{kV}$, nitrogen sheath gas flow rate of 10 units, capillary temperature set at $320^{\circ} \mathrm{C}$, and an AGC target set to $3 \mathrm{e} 6$. Samples were analyzed at a resolution of 140,000 in full scan mode. The scan window included 85 to $800 \mathrm{~m} / \mathrm{z}$ units from 0 to $9 \mathrm{~min}$ and 110 to $1000 \mathrm{~m} / \mathrm{z}$ units from 9 to $25 \mathrm{~min}$. Bile acids were analyzed by the same UPLC-HRMS instrument and column that was used as in the metabolomics analysis. The column compartment was kept at $40^{\circ} \mathrm{C}$ and the flow rate was kept at $300 \mu \mathrm{L} / \mathrm{min}$. Mobile phase composition was $0.1 \%$ formic acid in water labeled as solvent $A$ and $0.1 \%$ 
formic acid in acetonitrile. The mobile phase gradient consisted of: $0 \%$ to $100 \%$ B from 0 to $13 \mathrm{~min}, 100 \% \mathrm{~B}$ from 13 to $14 \mathrm{~min}, 100 \%$ to $0 \% \mathrm{~B}$ from 14 to $14.5 \mathrm{~min}$, 0\% B from $14.5 \mathrm{~min}$ to $20.5 \mathrm{~min}$. Eluent from this method was introduced into the mass spectrometer via a heated electrospray ionization (HESI) source also set to negative mode. Instrument settings include: spray voltage of $4.2 \mathrm{kV}$, nitrogen sheath gas flow of 25 units, capillary temperature set at $300^{\circ} \mathrm{C}$, and a AGC set to 3e6. Samples were analyzed at a resolution of 140,000 in full scan mode. The scan window was from $150-1000 \mathrm{~m} / \mathrm{z}$ units.

The collected data for each tissue was normalized by tissue weight (small intestine and ceca) or volume (plasma) followed by median normalization. Tissue data was then formatted for and analyzed with MetaboAnalyst v4.0 (http://www.metaboanalyst.ca/MetaboAnalyst/faces/home.xhtml), an online tool for metabolomic analysis ${ }^{160-163}$. Using the Statistical Analysis tool, PCA plots and heatmaps were generated for the untargeted metabolomics screen; for the heatmaps, time point groups were collapsed using group averages, and Ward's method was used for the clustering algorithm along with a Euclidean distance measure; relative abundance data was autoscaled to account for metabolites with very low or very high abundances. For bile acid heatmaps, neither the samples or features were clustered. The Enrichment Analysis tool was used to identify enriched pathways between Tac and CR mouse cecal samples at day 0 p.i. The library for analysis was the Pathway-associated Metabolite Sets and only metabolite sets containing at least 2 compounds were used. 


\section{Gut Microbiota Analysis:}

Mouse ceca and fecal pellets were extracted and flash frozen in liquid nitrogen followed by storage at $-80^{\circ} \mathrm{C}$. DNA was extracted using the QIAamp PowerFecal DNA kit (QIAGEN, Germantown, MD) according to the manufacturer's instructions. DNA samples were then shipped overnight on ice packs to either the Integrated Microbiome Resource within the Centre for Comparative Genomics and Evolutionary Bioinformatics at Dalhousie University (IMR-CGEB, Halifax, NS, Canada) or the Genome Technology Access Center at Washington University (GTAC, St. Louis, MO) for sequencing. Samples submitted to the IMR-CGEB were amplified using primers targeting the V6-V8 hypervariable regions. Sequencing was done on an Illumina MiSeq with 300bp paired-end reads. Sequence analysis was done using the Microbiome Helper pipeline, which provides wrapper scripts for common bioinformatics tools ${ }^{164}$. Briefly, sequences were inspected with FastQC v0.11.5; the paired-end reads were stitched together with PEAR v0.9.6 and filtered with BBMap v37.24 with a quality score cutoff of $30^{165-167}$. The filtered FASTQ files were converted to FASTA using the FASTX toolkit v0.0.13.2 and chimeras were removed with VSEARCH v1.11.1168,169. QIIME v1.9.1 was then run for open reference OTU picking using SortMeRNA v2.0 and SUMACLUST v1.0.20 for reference picking and de novo OTU picking; alignment was done using PyNAST v1.2.2 and Greengenes 13_8170-174. The resulting OTU table was cumulative sum scaled 
(CSS). Analyses were done within QIIME to produce taxa plots as well as calculate alpha diversity and Bray-Curtis beta diversity.

Samples submitted to the GTAC were amplified using a novel approach that consists of 12 primer pairs that span portions of all 9 of the 16S rRNA hypervariable regions ${ }^{175}$. Upon receiving the OTU table from GTAC, it was CSS normalized and analyzed within QIIME to produce taxa plots and calculate alpha diversity and Bray-Curtis beta diversity. Both the map file and OTU table used for analyses are included as supplementary data as Additional Files 8 and 9 , respectively.

PICRUSt was used to predict the functional capacity of the samples sent to the IMR-CGEB ${ }^{152}$. The OTU table was filtered to remove de novo OTUs to produce a compatible OTU table. After filtering, the OTU table was uploaded to the Langille Galaxy server (http://galaxy.morganlangille.com/) running PICRUSt v1.1.1 for normalization and analysis. All analyses used KEGG Orthologs for functional predictions.

The joint analysis of the PICRUSt data and metabolite data utilized MetaboAnalyst's Network Explorer tool. The KOs and metabolites were added as lists without fold changes for the day 0 time points in Tac and CR mice. The mode of analysis used the KEGG Global Metabolic Network and the table containing the significantly enriched pathways was downloaded.

\section{Statistical Analysis:}


Statistical analyses were performed using GraphPad Prism 7 software (GraphPad Software, La Jolla, CA, USA); the alpha value for each analysis was set at 0.05 . Specific analyses are described in figure legends. For area under the curve (AUC) parasite burden analyses, the trapezoidal rule was used:

$$
\operatorname{AUC}_{(\mathrm{t} 1-\mathrm{t}-\text { last })}=\Sigma\left(\mathrm{p}_{\mathrm{i}}+\mathrm{p}_{\mathrm{i}+1}\right)^{*}\left(\mathrm{t}_{\mathrm{i}+1}-\mathrm{t}_{\mathrm{i}}\right) / 2
$$

where " $\mathrm{p}$ " is percent parasitemia at the designated time point " $\mathrm{t}$ "123. 


\section{Figures and Figure Legends}

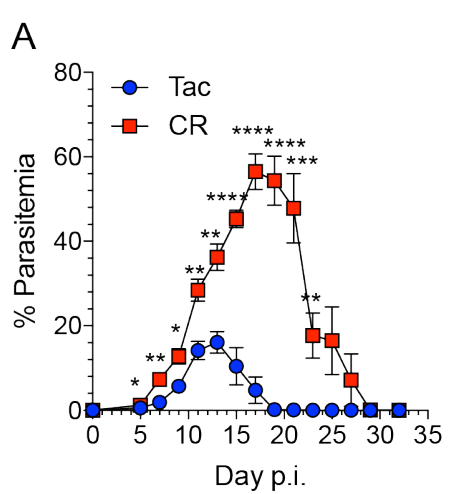

D

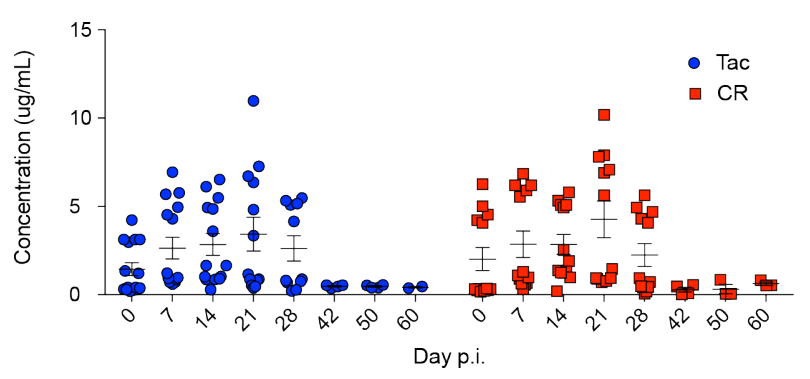

B

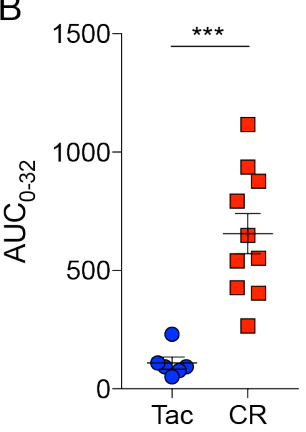

$\mathrm{E}$

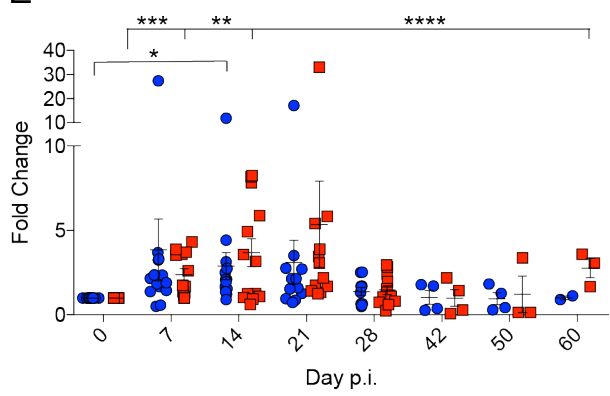

Figure 3-1. Susceptibility to Plasmodium infection varies between vendors. C57BL/6N mice from Taconic (Tac) and Charles River (CR) were infected with $P$. yoelii (Py). A. Percent parasitemia (percentage of red blood cells (RBCs) infected with Py) over the course of infection. Individual time points between Tac and CR were analyzed by unpaired two-tailed t-test. B. Area under the curve (AUC) analysis of percent parasitemia. Data were analyzed by unpaired two-tailed ttest. C. Percentage of weight lost post-infection (p.i.). Individual time points were analyzed by unpaired two-tailed t-test. D. FITC-dextran concentrations in serum over the course of infection. E. Data from panel D normalized to the Day 0 time point to show fold changes. All data were analyzed by unpaired two-tailed t-test. A-C. Data (mean $\pm S E$ ) are cumulative results ( $n=3-5$ mice/group) of two experiments. D-E. Data (mean \pm SE) are cumulative results of 3 experiments 
( $n=4-5$ mice/group/experiment). ${ }^{*} p<0.05 ;{ }^{* \star} p<0.01 ;{ }^{* \star *} p<0.001 ;{ }^{* \star \star *} p<$ 0.0001
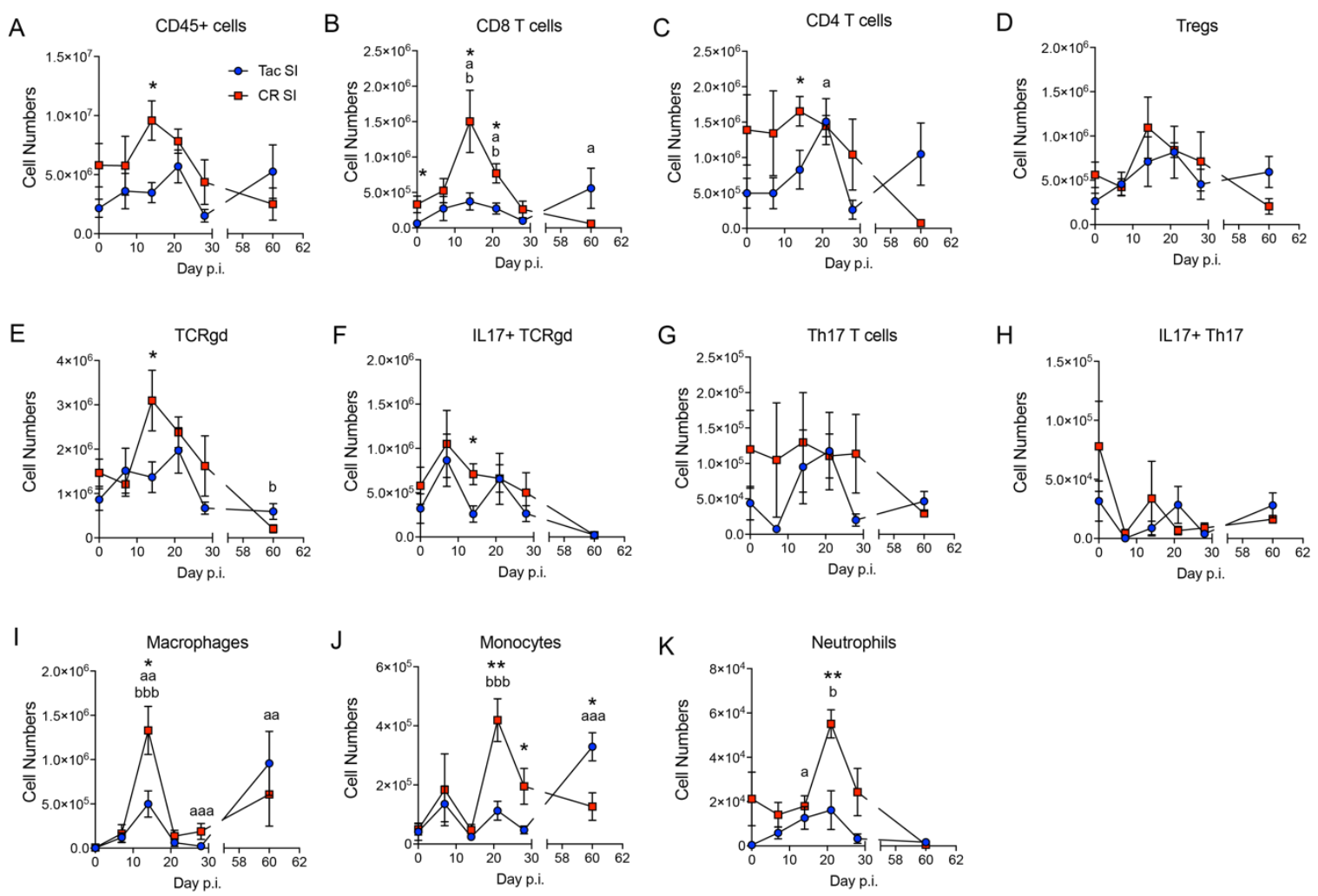

Figure 3-2. Small intestine lamina propria immune system changes during Py infection. Total cell numbers of A. CD45+ cells, B. CD8+ T cells, C. CD4+ T cells, D. Tregs, E. Gamma delta T cells (TCR $\gamma \delta)$, F. IL17+ TCR $\gamma \delta$ G. Th17 cells, H. IL17+ Th17 cells, I. Macrophages, J. Monocytes, and K. Neutrophils. Each time point was compared by one-way ANOVA with Tukey's post-hoc multiple comparison test. Data (mean $\pm S E$ ) are cumulative results of 2 experiments (3 mice/group/experiment). 1 symbol, $p<0.05 ; 2$ symbols, $p<0.01 ; 3$ symbols, $p<$ $0.001 ; 4$ symbols, $\mathrm{p}<0.0001 .{ }^{*}=$ Tac and CR SI comparisons; $\mathrm{a}=\mathrm{Tac} \mathrm{SI}$ comparisons to Day 0; b = CR SI comparisons to Day 0 . 


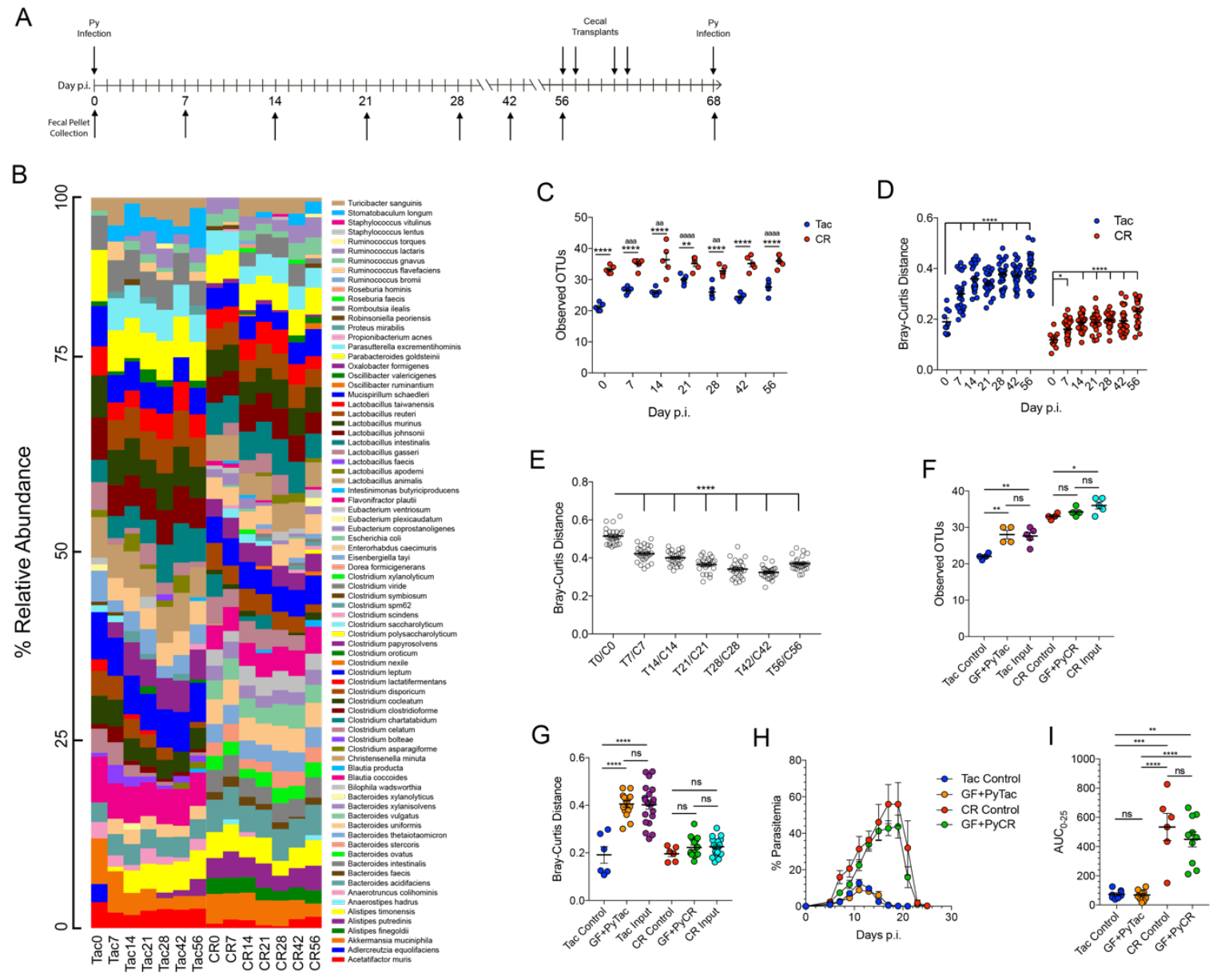

Figure 3-3. Gut bacterial community changes occurring post-Py infection do not change susceptibility to future Py infections. A. Experimental design of time course and cecal transplants along with fecal pellet collection times. B. Relative taxonomic abundance of bacterial species during Py infection. C. Alpha diversity (sample richness) between Tac and CR mice during infection using observed OTUs. Data were analyzed with a repeated measures two-way ANOVA with Dunnett's post-hoc multiple comparison test for comparisons to Day 0 p.i. and Sidak's post-hoc multiple comparison test to compare Tac and CR diversity at each time point. D. Beta diversity (sample dissimilarity) between Tac and CR 
mice during Py infection using the Bray-Curtis distance metric; each time point is compared to the respective Day 0 time point. Data were analyzed by one-way ANOVA with Dunnett's post-hoc multiple comparison test. E. Matched beta diversity comparisons between Tac and CR mice at each time point. Data were analyzed by one-way ANOVA with Dunnett's post-hoc multiple comparison test. F. Bacterial community alpha diversity from fecal pellets taken from mice receiving cecal contents. Data were analyzed by unpaired two-tailed t test. G. Bacterial community beta diversity from fecal pellets taken from mice receiving cecal contents. The comparisons are Tac control vs Tac control, GF+PyTac vs Tac control, CR control vs CR control, and GF+PyCR vs CR control. Data were analyzed by unpaired two-tailed t test. H. Parasitemia of GF mice gavaged with post-Py Tac or post-Py CR cecal contents along with controls. I. AUC of the parasite burdens shown in panel H. Data were analyzed by unpaired two-tailed $t$ test. Data $(m e a n \pm S E)$ in panels $\mathbf{B}-\mathbf{G}$ are from one experiment (4-5 mice per group); panels $\mathbf{H}-\mathbf{I}$ are cumulative results of 2 experiments (4-5 mice/group/experiment). 1 symbol, $p<0.05 ; 2$ symbols, $p<0.01 ; 3$ symbols, $p<$ $0.001 ; 4$ symbols, $\mathrm{p}<0.0001 ; \mathrm{ns}=$ not significant. ${ }^{*}=$ Tac and $\mathrm{CR}$ comparisons; $a=$ Tac comparisons with Day 0; $b=C R$ comparisons with Day 0. 

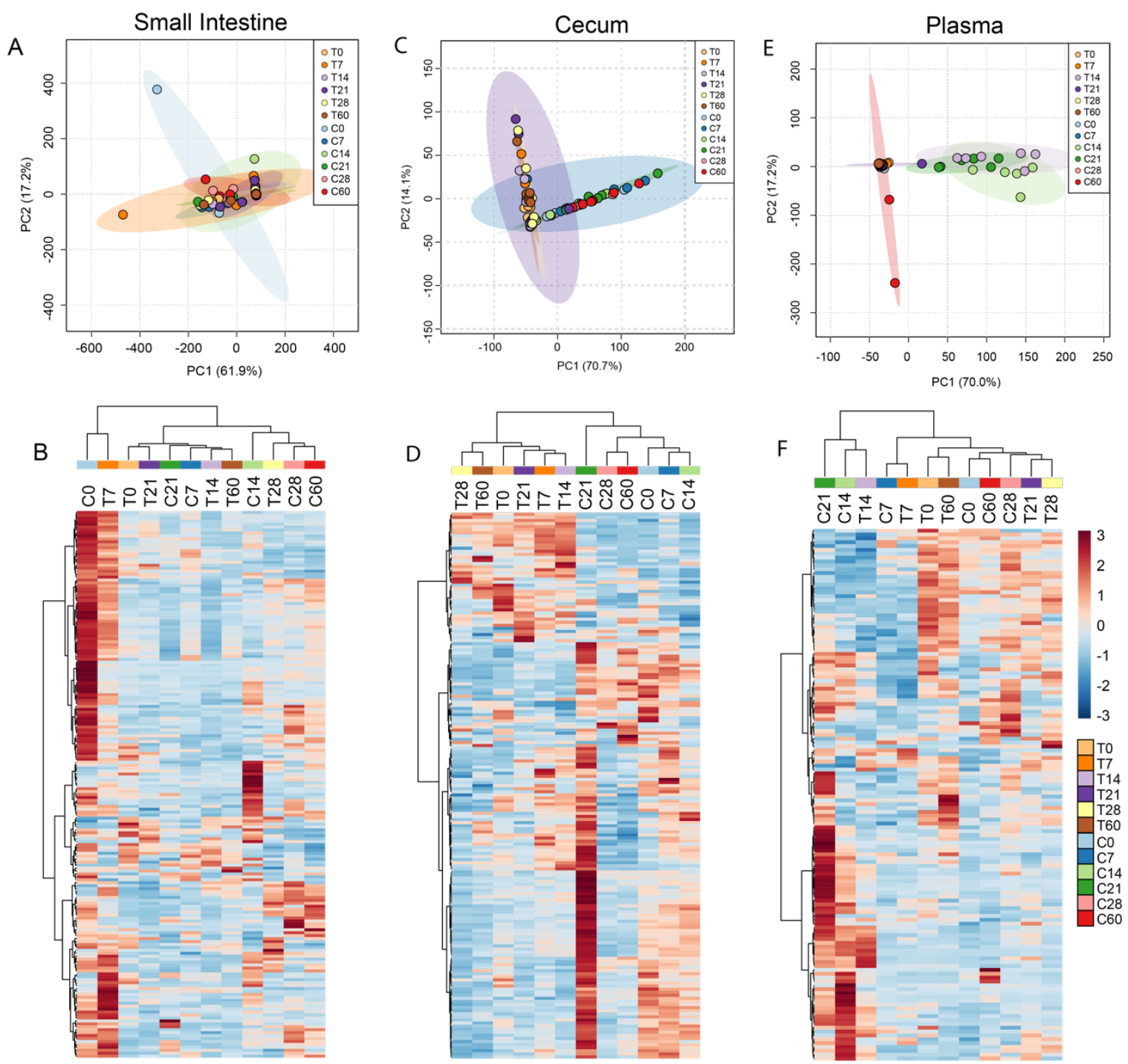

Figure 3-4. Metabolite profiles in selected tissues during Py infection.

Principal Component Analysis (PCA) plot showing similarity of metabolite profiles in Tac and CR samples in the small intestine A., cecum C., and plasma $\mathbf{E}$. Ellipses contain the $90 \%$ confidence area of each group. Metabolite profile heatmap from the small intestine B., cecum D., and plasma F. Sample group averages and metabolites are clustered using Ward's method and Euclidean distance measure. Data are the cumulative results of 2 experiments (3 
mice/group/experiment). Scale bar represents scaled relative abundance of metabolites.

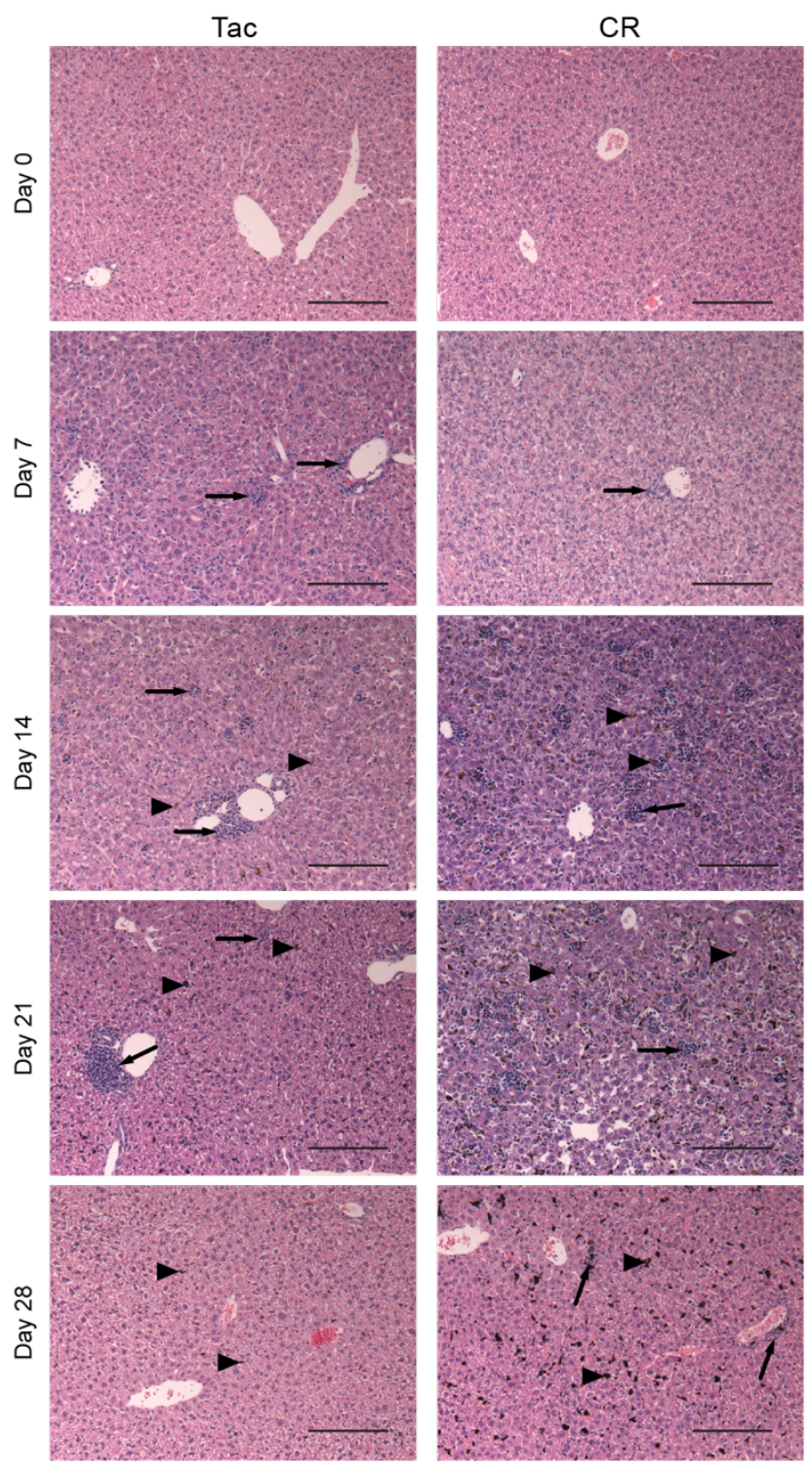

Figure 3-5. Severity of liver damage following Py infection correlates with both parasitemia burden and kinetics. Histology of representative livers (3 mice/group/time point) stained with H\&E in naive and Py-infected mice. Scale bar 
$=100 \mu \mathrm{m} ;$ magnification $=20 \mathrm{x}$. Arrowheads indicate hemozoin deposition; arrows indicate immune cell infiltration.

A

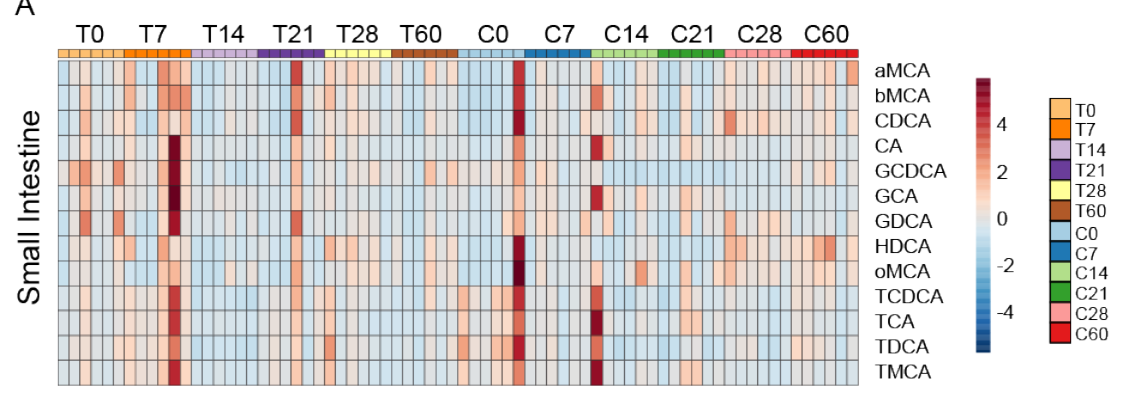

B

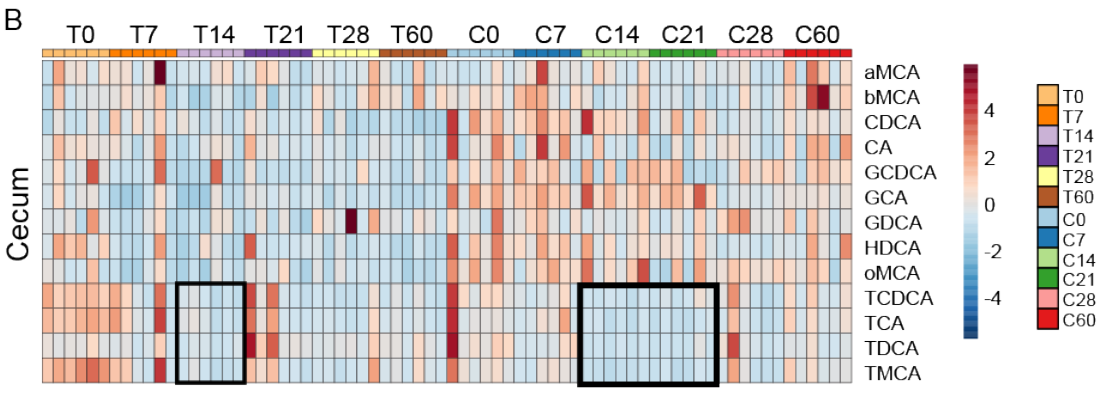

$\begin{array}{lllllllllllll}\text { C } & \text { T0 } & \text { T7 } & \text { T14 } & \text { T21 } & \text { T28 } & \text { T60 } & \text { C0 } & \text { C7 } & \text { C14 } & \text { C21 } & \text { C28 } & \text { C60 }\end{array}$

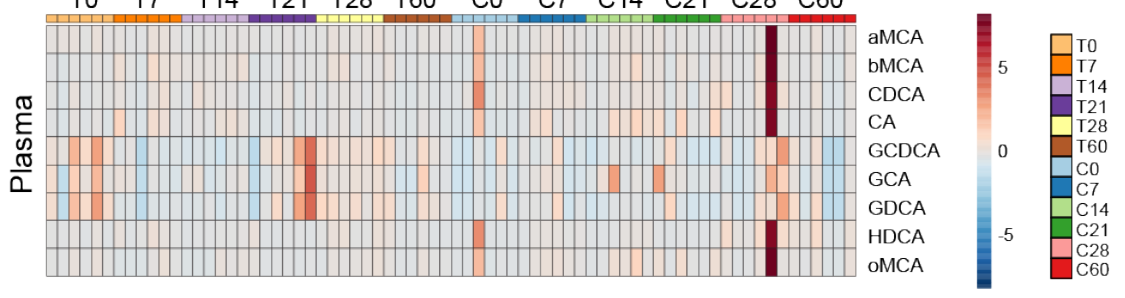

Figure 3-6. Severity of Py infection correlates with loss of specific cecal

bile acids. A. Small intestine bile acid heatmap. B. Cecal bile acid heatmap. C.

Plasma bile acid heatmap. Data are cumulative over 2 experiments (3 mice/group/experiment). Scale bars indicate scaled relative abundance of bile acids. aMCA = alpha-muricholic acid; bMCA = beta-muricholic acid; $\mathrm{CDCA}=$ chenodeoxycholic acid; $\mathrm{CA}=$ cholic acid; GCDCA = glycochenodeoxycholic acid; GCA = glycocholic acid; GDCA = glycodeoxycholic acid; HDCA = hyodeoxycholic 
acid; oMCA = omega-muricholic acid; TCDCA = taurochenodeoxycholic acid; TCA = taurocholic acid; TDCA = taurodeoxycholic acid; $\mathrm{TMCA}=$ tauromuricholic acid.

A

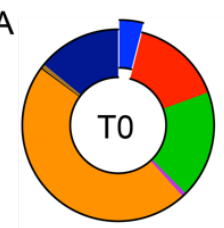

Total $=3.19774 \mathrm{E}+07$

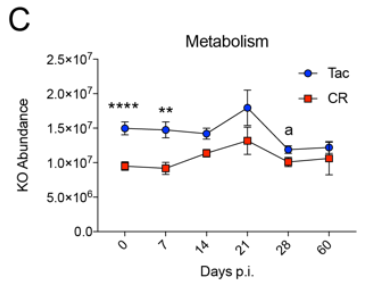

D
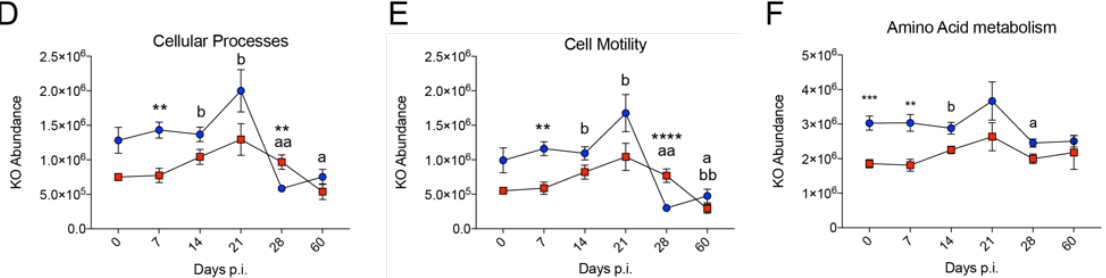

G Day 0 p.i. - Enrichment Overview (top 50)

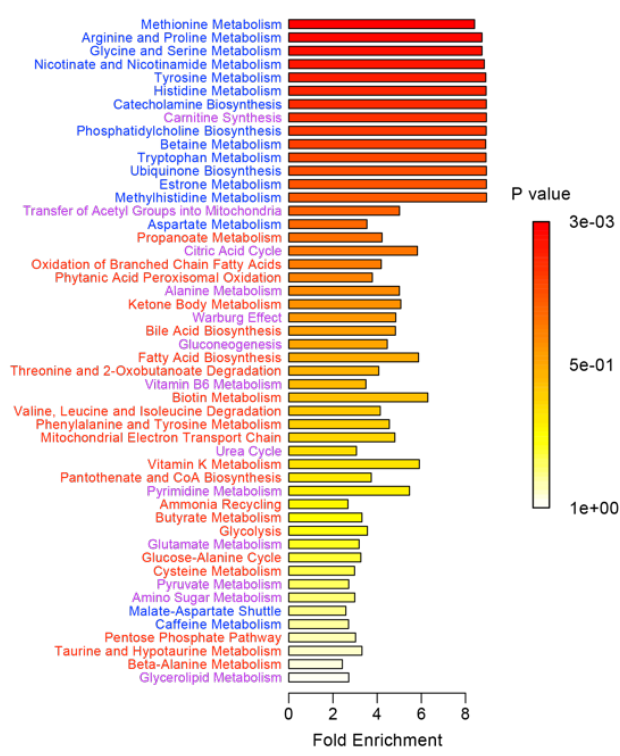

E
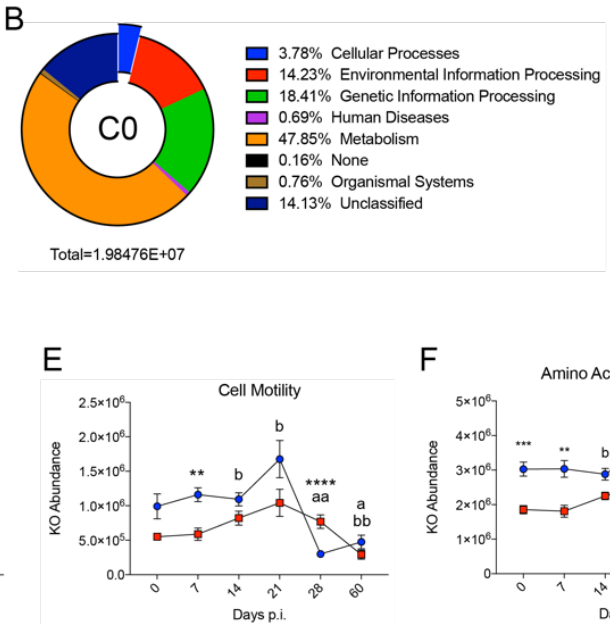

Total $=1.98476 \mathrm{E}+07$

$\mathrm{H}$

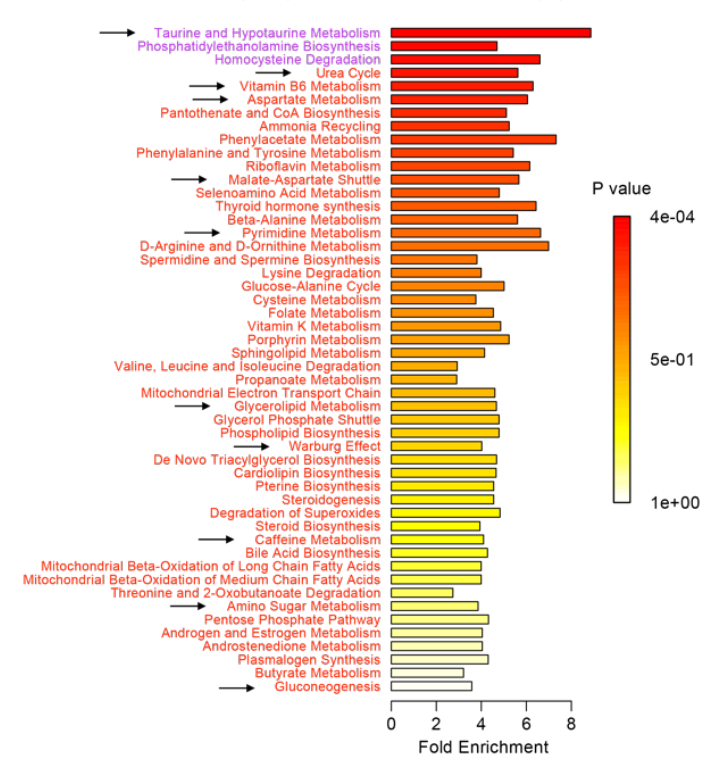

Figure 3-7. Severe Py infection increases the predicted functional capacity of the gut microbiota. A. KO proportions and total abundance in the Tac cecal microbiota at day 0 p.i. B. KO proportions and total abundance in the $\mathrm{CR}$ cecal 
microbiota at day 0 p.i. C. KO abundances during Py infection classified to the level 1 KEGG category "Metabolism" between Tac and CR microbiota. D. KO abundances during Py infection classified to the level 1 KEGG category "Cell Processes" between the Tac and CR microbiota. E. KO abundances during Py infection classified to the level 2 KEGG category "Cell Motility" between the Tac and CR microbiota. F. KO abundances of the "Amino Acid metabolism" category during Py infection. G. Cecal metabolite pathway enrichment between Tac and CR mice at day 0 p.i. Blue means a pathway is enriched in Tac while red is enriched in CR; purple indicates enrichment in both. H. Cecal metabolite pathway enrichment at day 21 p.i. Arrows represent pathways that were enriched in Tac at ay 0 p.i. but are enriched in CR at day 21 p.i. and vice versa. Data in C-F were analyzed by unpaired two-tailed t-test. G-H. Data were analyzed using Metaboanalyst's Enrichment Analysis based on the globaltest algorithm and are Holm-Bonferroni adjusted. Data (mean \pm SE) originate from Figure S3 samples and are cumulative results ( $n=2-3$ mice/group/experiment) of two experiments. 1 symbol, $p<0.05 ; 2$ symbols, $p<0.01 ; 3$ symbols, $p<0.001 ; 4$ symbols, $p<$ 0.0001. ${ }^{*}=$ Tac and CR comparisons; $a=$ Tac comparisons with Day 0; $b=C R$ comparisons with Day 0. 


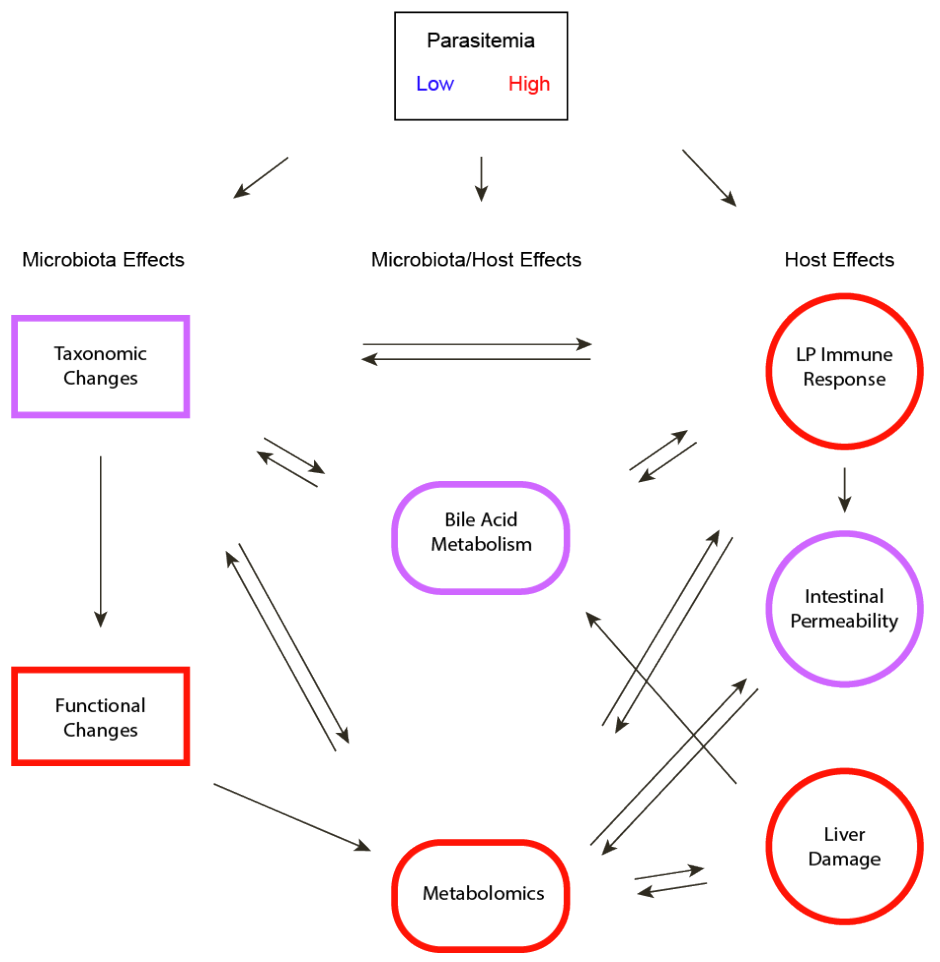

Figure 3-8. Schematic of the different host and microbiota factors. Arrows represent the ability of one factor to influence another. Colors represent differential effects driven by low or high parasitemia. 

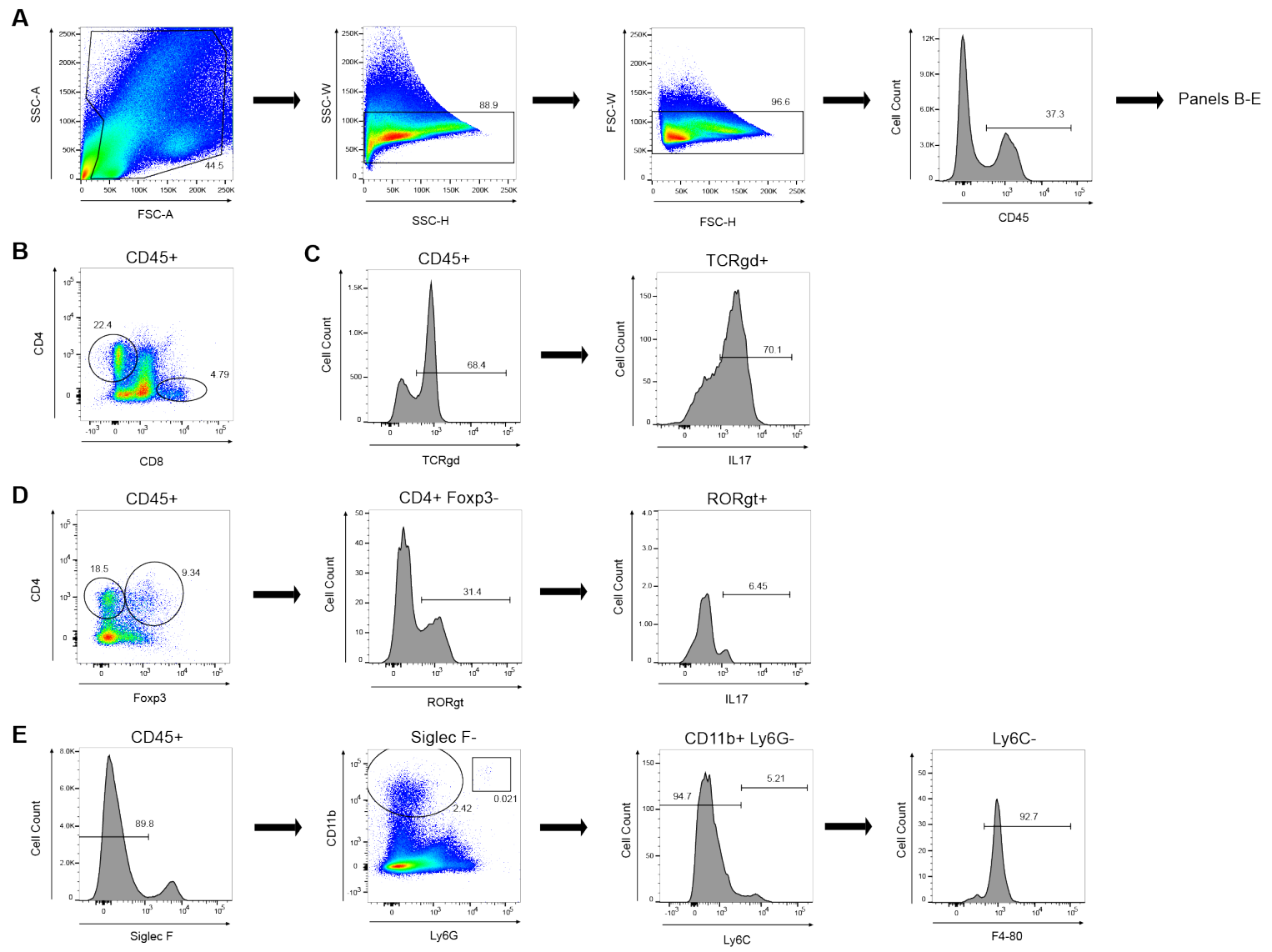

Figure 3-9. Gating strategies from the LP of mouse SI and LI. A. Gating strategy of single cell suspension and CD45+ cells. B. Gating strategy for CD4+ and CD8+ T cells. C. Gating strategy for TCR $\gamma \delta$ T cells and IL17+ TCR $\gamma \delta$ T cells. D. Gating strategy for Tregs, Th17 T cells, and IL17+ Th17 T cells. E. Gating strategy for neutrophils, monocytes, and macrophages. 

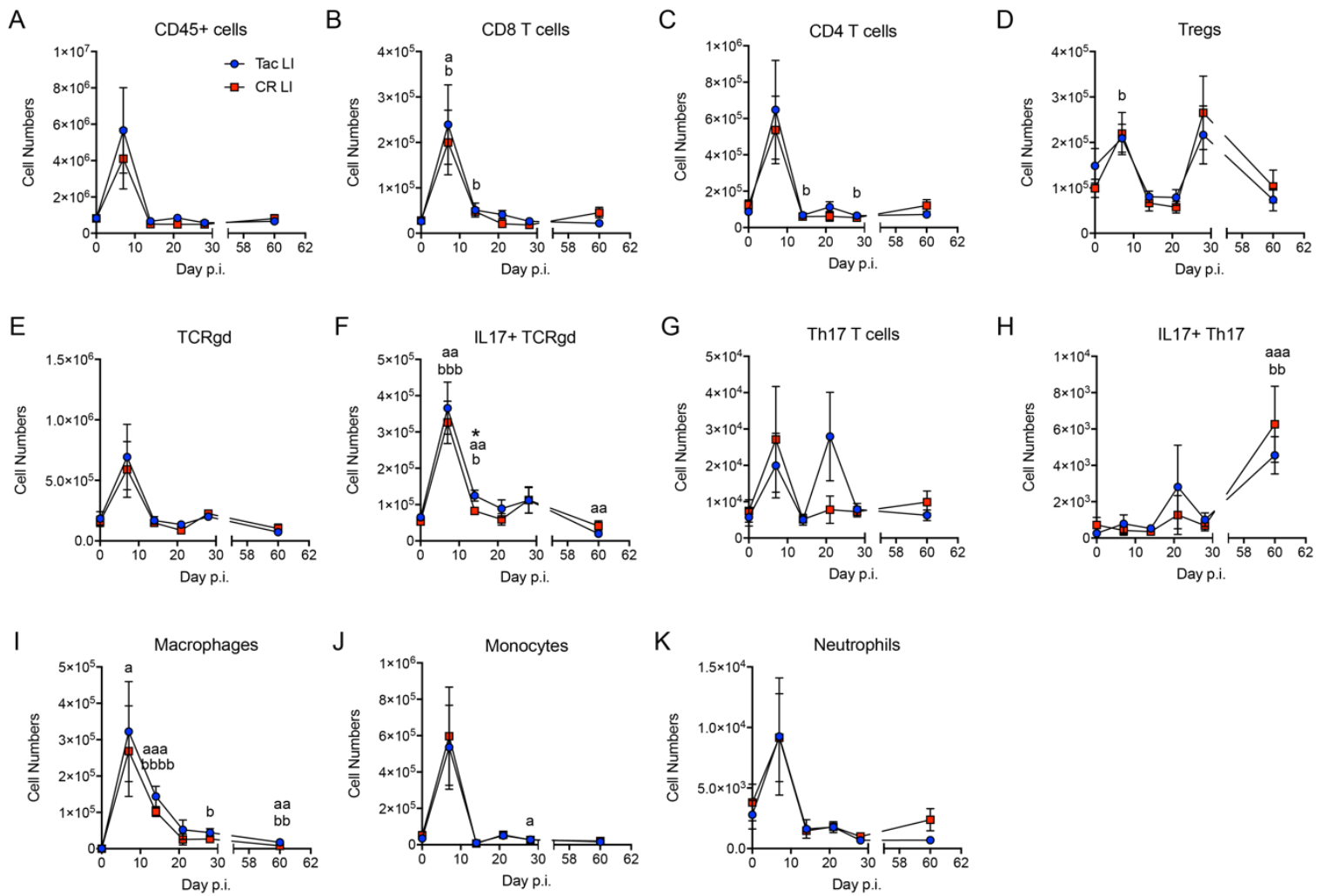

Figure 3-10. Large intestine lamina propria immune system changes during Py infection. Total cell numbers of A. CD45+ cells, B. CD8+ T cells, C. CD4+ T cells, D. Tregs, E. Gamma delta T cells (TCR $\gamma \delta)$, F. IL17+ TCR $\gamma \delta$ G. Th17 cells, H. IL17+ Th17 cells, I. Macrophages, J. Monocytes, and K. Neutrophils. Each time point was compared by one-way ANOVA with Tukey's post-hoc multiple comparison test. Data (mean \pm SE) are cumulative results of 2 experiments ( 3 mice/group/experiment). 1 symbol, $p<0.05 ; 2$ symbols, $p<0.01 ; 3$ symbols, $p<$ $0.001 ; 4$ symbols, $\mathrm{p}<0.0001 .{ }^{*}=$ Tac and CR LI comparisons; $\mathrm{a}=\mathrm{Tac} \mathrm{LI}$ comparisons to Day 0; b = CR LI comparisons to Day 0. 

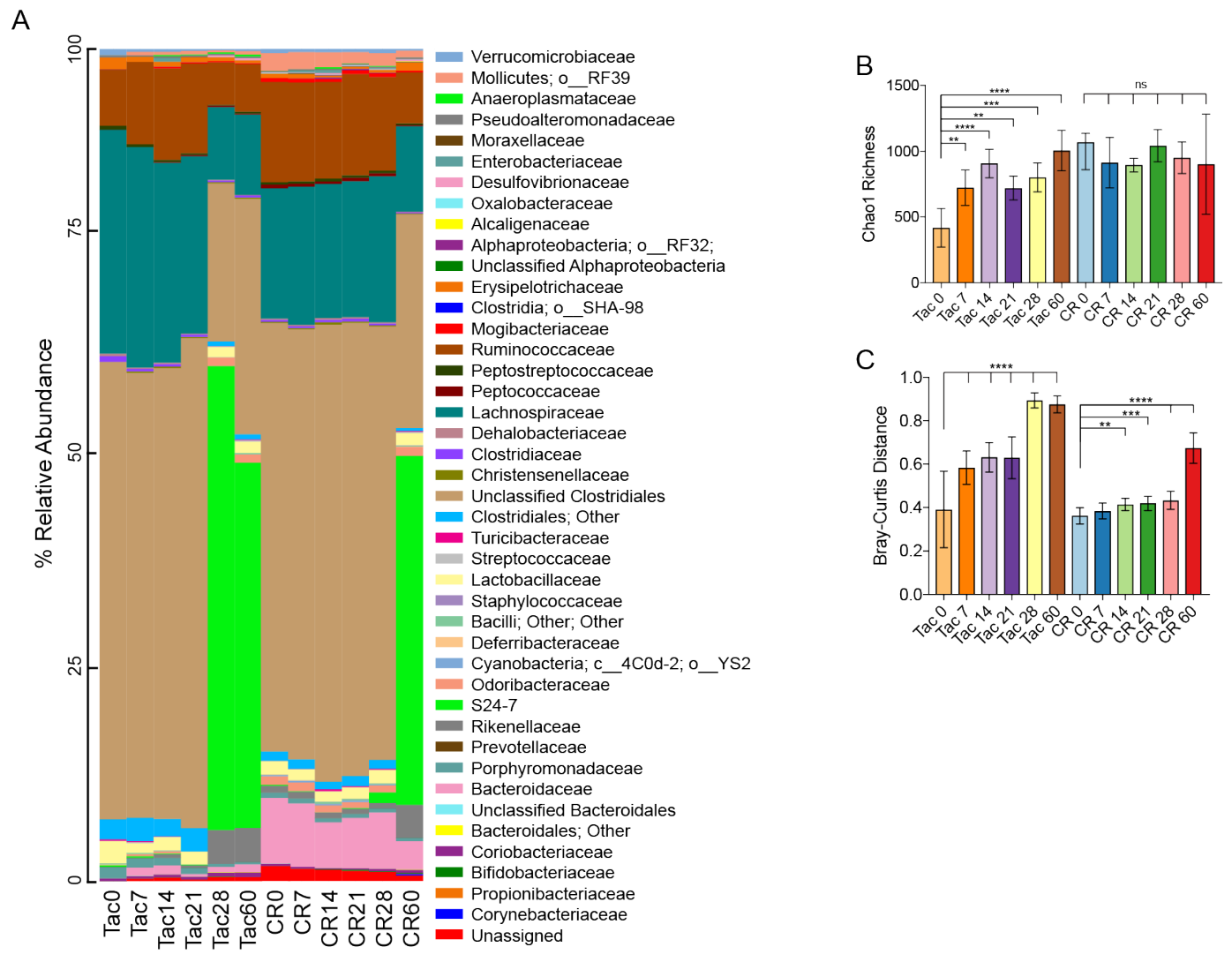

Figure 3-11. The composition of gut bacteria populations changes after

clearance of Py. A. Relative taxonomic abundance of bacterial families during

Py infection. $\mathrm{C}=$ class, $\mathrm{O}=$ order. $\mathrm{B}$. Alpha diversity (sample richness) between

Tac and CR mice during infection using the Chao1 metric. C. Beta diversity (sample dissimilarity) between Tac and CR mice during Py infection using the Bray-Curtis distance metric; each time point is compared to the respective Day 0 time point. Data in B-C were analyzed by one-way ANOVA with Dunnett's posthoc multiple comparison test. Data (mean $\pm \mathrm{SE}$ ) are cumulative results of 2 experiments (2-3 mice/group/experiment). ${ }^{*} \mathrm{p}<0.05 ;{ }^{* \star} \mathrm{p}<0.01 ;{ }^{* \star *} \mathrm{p}<0.001$; ${ }^{* * *} p<0.0001 ; n s=$ not significant 

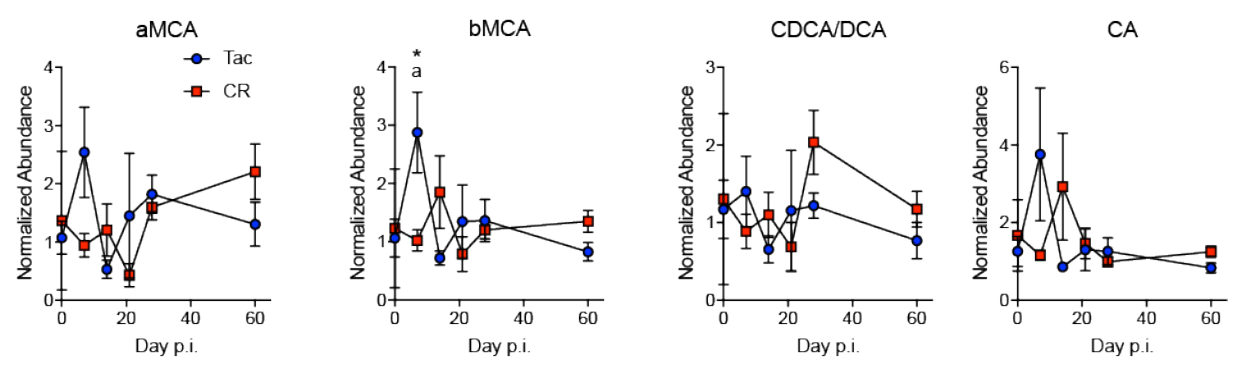

GCDCA

GCA
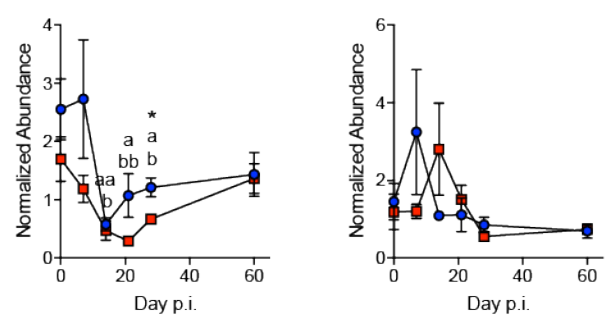

GDCA

HDCAUUDCA

OMCA

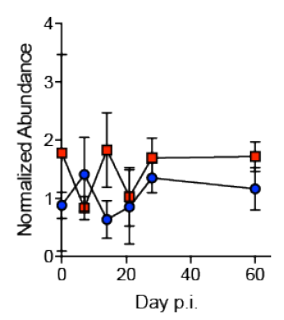

TCDCA
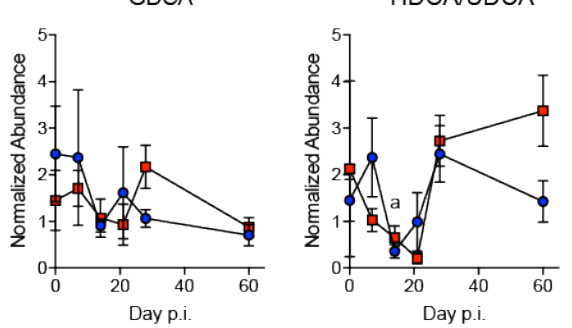

TCA
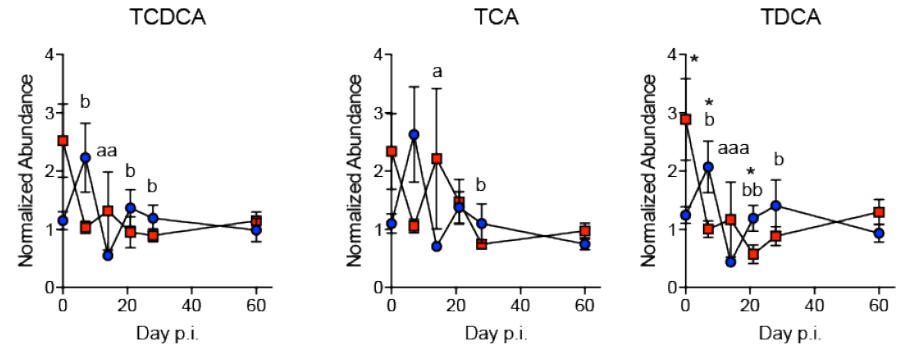

aTMCA

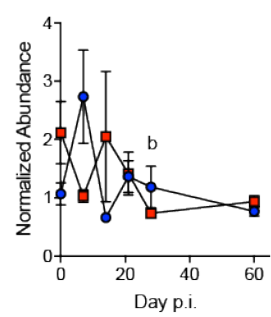

Figure 3-12. Small intestine bile acid profiles over Py infection. aMCA = alpha-muricholic acid; $\mathrm{bMCA}=$ beta-muricholic acid; $\mathrm{CDCA}=$ chenodeoxycholic acid; $\mathrm{CA}=$ cholic acid; $\mathrm{GCDCA}=$ glycochenodeoxycholic acid; $\mathrm{GCA}=$ glycocholic acid; GDCA = glycodeoxycholic acid; HDCA = hyodeoxycholic acid; oMCA = omega-muricholic acid; TCDCA = taurochenodeoxycholic acid; TCA = taurocholic acid; TDCA = taurodeoxycholic acid; TMCA = tauromuricholic acid. Data were analyzed by unpaired t-test. Data (mean $\pm S E)$ are cumulative results 
( $n=3$ mice/group/experiment) of two experiments. 1 symbol, $p<0.05 ; 2$ symbols, $\mathrm{p}<0.01 ; 3$ symbols, $\mathrm{p}<0.001 ; 4$ symbols, $\mathrm{p}<0.0001 .{ }^{*}=$ Tac and $\mathrm{CR}$ comparisons; $\mathrm{a}=$ Tac comparisons with Day $0 ; \mathrm{b}=\mathrm{CR}$ comparisons with Day 0 .
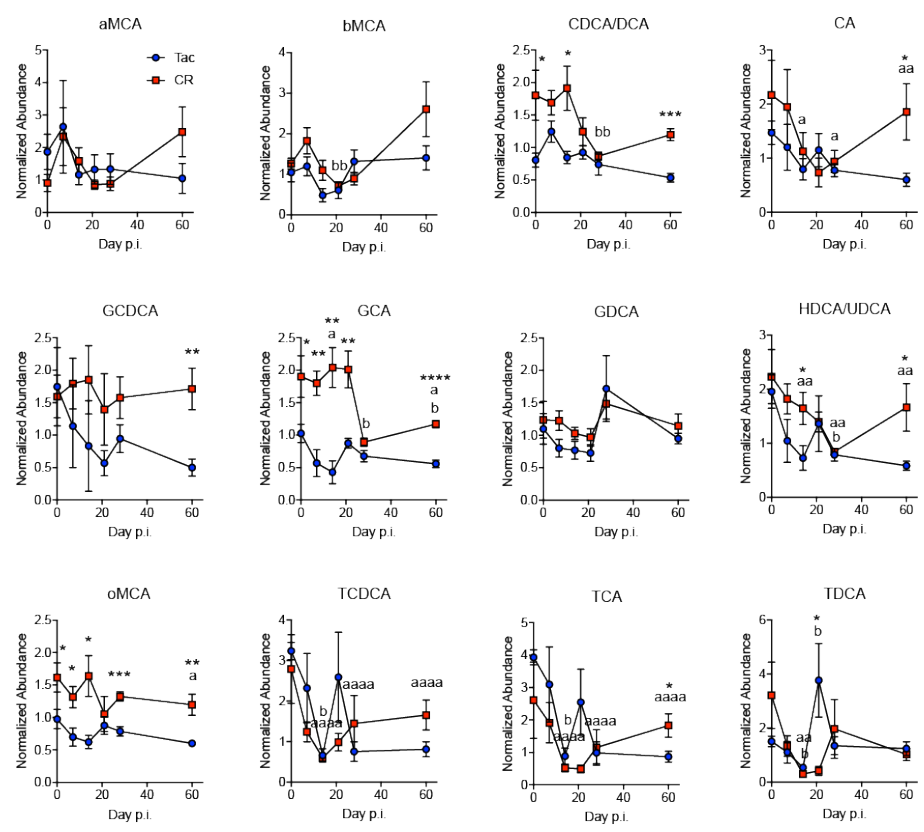

aTMCA

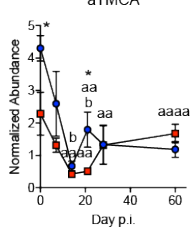

Figure 3-13. Cecal bile acid profiles over Py infection. aMCA = alpha-

muricholic acid; $\mathrm{bMCA}=$ beta-muricholic acid; $\mathrm{CDCA}=$ chenodeoxycholic acid; $\mathrm{CA}=$ cholic acid $; \mathrm{GCDCA}=$ glycochenodeoxycholic acid; $\mathrm{GCA}=$ glycocholic acid; GDCA = glycodeoxycholic acid; HDCA = hyodeoxycholic acid; oMCA = omega-muricholic acid; TCDCA = taurochenodeoxycholic acid; TCA = taurocholic acid; TDCA = taurodeoxycholic acid; TMCA = tauromuricholic acid. Data were analyzed by unpaired t-test. Data (mean $\pm S E)$ are cumulative results 
( $n=3$ mice/group/experiment) of two experiments. 1 symbol, $p<0.05 ; 2$ symbols, $\mathrm{p}<0.01 ; 3$ symbols, $\mathrm{p}<0.001 ; 4$ symbols, $\mathrm{p}<0.0001 .{ }^{*}=$ Tac and $\mathrm{CR}$ comparisons; $\mathrm{a}=$ Tac comparisons with Day $0 ; \mathrm{b}=\mathrm{CR}$ comparisons with Day 0 .
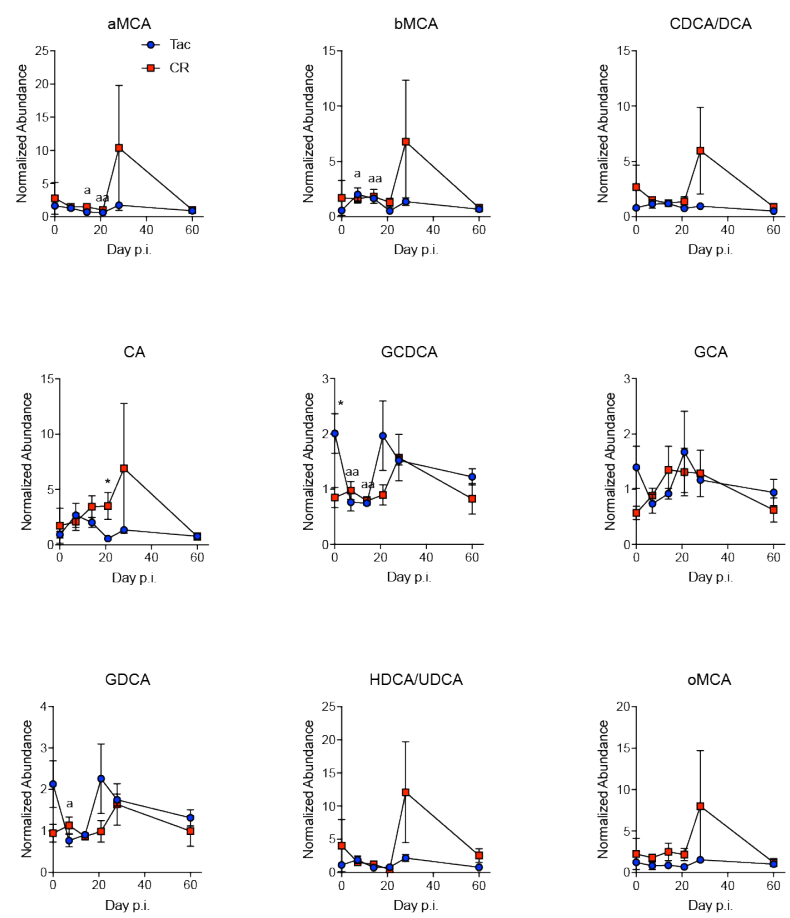

Figure 3-14. Plasma bile acid profiles over Py infection. aMCA = alphamuricholic acid; bMCA = beta-muricholic acid; CDCA = chenodeoxycholic acid; $\mathrm{CA}=$ cholic acid GCDCA = glycochenodeoxycholic acid; $\mathrm{GCA}=$ glycocholic acid; GDCA = glycodeoxycholic acid; HDCA = hyodeoxycholic acid; oMCA = omega-muricholic acid. Data were analyzed by unpaired t-test. Data (mean $\pm S E)$ are cumulative results ( $\mathrm{n}=3$ mice/group/experiment) of two experiments. 1 symbol, $\mathrm{p}<0.05 ; 2$ symbols, $\mathrm{p}<0.01 ; 3$ symbols, $\mathrm{p}<0.001 ; 4$ symbols, $\mathrm{p}<$ 0.0001. * $=$ Tac and CR comparisons; $a=$ Tac comparisons with Day 0; $b=C R$ comparisons with Day 0. 


\title{
CHAPTER 4
}

\section{ORAL ADMINISTRATION OF CLINICALLY RELEVANT ANTIMALARIAL DRUGS DOES NOT MODIFY THE MURINE GUT MICROBIOTA}

\author{
Malaria is an infectious disease responsible for the death of around \\ 450,000 people annually. As an effective vaccine against the parasite that \\ causes malaria is not available, antimalarial drug treatments are critical in fighting \\ the disease. Previous data has shown that the gut microbiota is important in \\ modulating the severity of malaria. Although it is well appreciated that antibiotics \\ substantially alter the gut microbiota, it is unknown how antimalarial drugs impact \\ the microbiota. We show here that the two commonly used artemisinin \\ combination therapies of artesunate plus amodiaquine and artemether plus \\ lumefantrine do not change the gut microbiota. The overall relative species \\ abundance and alpha diversity remained stable after treatment, while beta \\ diversity analysis showed minimal changes after cessation of drug treatment, \\ which were transient and quickly returned to baseline. Taken together, \\ antimalarial drug administration does not affect the gut microbiota.
}




\section{Introduction}

Plasmodium is a significant global pathogen which infected 216 million people and caused 445,000 deaths in 2016; in sub-Saharan Africa, children under the age of 5 years old are the most vulnerable ${ }^{1}$. Currently, there is no effective vaccine against Plasmodium. Although there is growing resistance to frontline antimalarial drugs, particularly in Southeast Asia ${ }^{124,176}$, these drugs are still critical for treating those infected with Plasmodium. The gold standard for malaria treatment is artemisinin combination therapy (ACT). Artemisinin itself was originally isolated from the plant Artemisia annua in 1972 and has several semi-synthetic derivatives, including artemether, artesunate, and dihydroartemisinin ${ }^{177}$. Pharmacologically, these derivatives are more effective at clearing Plasmodium parasites than other anti-malarial drugs but tend to have shorter half-lives ${ }^{178}$. Consequently, artemisinin derivatives are usually combined with another anti-malarial drug that has a longer half-life to create an ACT to ensure continual parasite eradication and reduce instances of resistance to antimalarial drugs. Two of the most common ACTs are artemether combined with lumefantrine and artesunate combined with amodiaquine; these are commonly given orally but can also be administered intravenously, rectally, or intramuscularly20.

The mechanism of action for artemisinin and its derivatives has recently been shown to involve cellular damage in Plasmodium-infected red blood cells (iRBCs) along with inhibition of the proteasome; this leads to endoplasmic 
reticulum stress and the buildup of damaged proteins ${ }^{55}$. In vitro experiments have also shown that artesunate can generate reactive oxygen species within iRBCs that lead to caspase activation and apoptosis 56 . The mechanisms for lumefantrine and amodiaquine are currently unknown, but it is suspected that both drugs interfere with Plasmodium's ability to detoxify heme ${ }^{54}$. Both types of mechanisms of action synergize to give ACTs their effectiveness in treating malaria.

Previous research has shown antibacterial effects of artemisinin and its derivatives on specific pathogens. Derivatives of artemisinin were shown to be differentially effective at killing various fungi and bacteria, including methicillinresistant Staphylococcus aureus (MRSA) ${ }^{57}$. Additionally, encapsulation of artemisinin with beta-cyclodextrin enhances its antibacterial capacity when tested against MRSA ${ }^{179}$. While modifying artemisinin and its derivatives increases the antibacterial efficacy, these modified derivatives are not used clinically. However, unmodified artemether and artemisinin have been shown to be relatively potent at killing Helicobacter pylori, which is a human pathobiont 57,58 .

The gut microbiota has been shown to be important for many aspects of health and disease, including malaria. We have previously shown in mice that the gut microbiota can modulate the severity of infection, and modifying the microbiota through something as simple as changing the diet was sufficient to make mice susceptible to severe malaria ${ }^{136}$. Whereas oral antibiotic treatment has profound effects on the gut microbiota, the impact of antimalarial treatment 
on the gut microbiota is unknown. Using a mouse model, we show that two commonly used ACTs do not substantially change the gut microbiota at clinical dosing.

\section{Results}

\section{Treatment with Clinically Relevant Doses of ACTs does not Change the Taxonomic Composition of the Murine Gut Microbiota}

To test our hypothesis, we used two common ACTs: artesunate combined with amodiaquine (AA) and artemether combined with lumefantrine (AL). Due to the hydrophobic nature of lumefantrine, AL was dissolved in olive oil $(O)$, while AA was dissolved in saline $(H)$. The ACTs and vehicle controls were given by oral gavage daily for three days, similar to clinical practice. Fecal pellets were collected before treatment (day 0 ) and at days $1,2,3,5,7,10,14$, and 21 posttreatment (p.t.) (Fig. 4-1A).

Overall, the relative abundance of bacterial species remains consistent over treatments when compared to the pooled, pretreatment day 0 samples (Fig. 4-1B). When the Bray-Curtis beta diversity is viewed in a PCoA plot and the samples are colored by time p.t., there is no appreciable separation between any of the time points, indicating high similarity (Fig. 4-1C). Whereas a separation between two populations can be seen in the panel displaying PC2 vs PC3 (Fig. 4-1C), this separation is not attributed to any of the treatments or time points but rather due to variability between the first and second experiments. The overall 
variability is small (12.16\%) and is explained by the presence or absence of a small handful of species in one experiment. Within each experiment, there is no discernable pattern between the different time points p.t. Similarly, when the samples are colored by treatment received (Fig. 4-1D), there are no major differences, indicating neither ACTs or vehicle treatments lead to major changes. Overall, when comparing the relative taxonomic abundances and beta diversity plots, there are no consistent patterns indicating changes due to antimalarial treatment.

\section{Antimalarial Treatments do not Affect the Overall Diversity of the Murine}

\section{Gut Microbiota}

Alpha diversity analysis (measured by the number of observed OTUs per sample) of samples from mice treated with $A A$ and the vehicle control, $H$, shows that the species composition does not change significantly over the course of the experiment (Fig 4-2A, B). For the beta diversity/dissimilarity comparisons, the day 0 p.t. within-group dissimilarity is compared to either the dissimilarity between the day 0 samples and the $\mathrm{H}$-treated mice or between the day 0 samples and the AA-treated mice, with higher beta diversity indicating greater dissimilarity. This comparison will identify time points in either the H- or AAtreated mice that become more or less similar to the pre-treatment day 0 bacterial communities following treatments, designated here as the "day 0 comparison". In this case, neither $\mathrm{H}$ nor AA caused the gut microbiota to change 
from the day 0 baseline (Fig. 4-2C). The day 0 comparisons are then compared between treatments at each time point; this "treatment comparison" identifies dissimilarities in the gut microbiotas between treated and untreated mice even if there are no significant differences in the day 0 comparisons. Even though mice treated with $\mathrm{H}$ or $\mathrm{AA}$ show no significant day 0 comparisons, there are significant differences between the treatment groups at days 7 and 10 p.t., with the $\mathrm{H}$ treatment having a modestly higher Bray-Curtis distance (Fig. 4-2C). The lack of significance of the day 0 comparisons at these time points indicates that the differences in treatment comparisons, while significant, are not biologically relevant. That these differences were not until 4 to 7 days post $\mathrm{H}$ - and $\mathrm{AA}$ treatment after the last treatments suggests that these differences were not attributed to treatments.

The next ACT examined was AL and its vehicle $\mathrm{O}$. The alpha diversity for mice receiving either vehicle or AL again showed that there were no significant changes to the number of species found in the gut microbiota during the experiment (Fig. 4-2D, E). The day 0 comparisons of beta diversity found that several of both $\mathrm{AL}$ - and O-treated samples have a greater dissimilarity to the day 0 composition than day 0 has internally at day 3 p.t. for both $A L(0.3468$ versus $0.2974 ; p=0.0011)$ and $O(0.3498$ versus $0.2974 ; p=0.0002)$, day 5 p.t. for $A L$ ( 0.3429 versus $0.2974 ; p=0.0032)$, and day 21 p.t. for $O$ ( 0.3552 versus 0.2974 ; $\mathrm{p}<0.0001$ ) (Fig. 4-2F). These differences indicate that there are significant, though modest, differences in the gut microbiota of these mice at the specified 
time points. Based on this data, it can be concluded that the treatments are contributing to the observed changes. However, with the increase in dissimilarity at day 3 for both $\mathrm{O}$ and $\mathrm{AL}$, it cannot be ruled out that the olive oil vehicle alone caused the difference. The treatment comparisons show significant differences between $O$ and $A L$ at days 5 and 21 p.t., with $A L$ samples having higher dissimilarity at day 5 p.t. compared to $\mathrm{O}$, and $\mathrm{O}$ samples having higher dissimilarity at day 21 p.t. (Fig. 4-2F). This is consistent with the previous findings from the day 0 comparison, as the time points that show significant differences in treatment dissimilarity are the same time points to be significantly different compared to day 0 . However, it must also be pointed out that these time points occur at least three days after the last AL treatment and 19 days after the last $\mathrm{O}$ treatment, which is considerably later when compared to previous data examining diet- or antibiotic-induced changes to the gut microbiota ${ }^{13,14}$. Additionally, these differences, at least for AL, appear to be transient, occurring only at the single time point. When the data is considered as a whole, there are several significant but minor differences in the microbiotas of mice treated with antimalarials, but no robust, long-term changes are seen.

\section{Discussion}

In this study, we have shown that treatment with common ACTs does not change the gut microbiota of mice. This is an important observation, given the widespread use of these antimalarials around the globe and the diverse effect of 
the gut microbiota on the host, including its ability to shape the severity of malaria ${ }^{136}$. Importantly, this work needs to be corroborated in humans, as mice and the murine gut microbiota are not perfect analogs of humans.

Since the gut microbiota does not change appreciably, it does not appear that the antimalarial treatments affect the microbiota directly, despite previous research on the antibacterial effects of artemisinin and its derivatives ${ }^{57,58}$. However, in the context of an inflammatory disease like malaria that has been shown to have an effect on the composition of the gut microbiota ${ }^{37,38}$, antimalarials may in fact protect the microbiota. In a model of hepatitis, artesunate treatment inhibited production of inflammatory cytokines such as interferon gamma and tumor necrosis factor alpha while driving production of the anti-inflammatory IL-10180. The cytokine modulation was driven by inhibition of the NF-kB signaling pathway, as artesunate enhanced phosphorylation of the NF-kB pathway and signaling components in liver tissue ${ }^{180}$. In macrophages, artesunate has also been shown to block autophagy-dependent activation due to LPS and thus downstream production of inflammatory cytokines ${ }^{181}$. With this in mind, artemisinin and its derivatives may not only eliminate malaria parasites during infection but also have secondary effects in maintaining host gut homeostasis.

There is still much that is unknown about artemisinin and its interactions with Plasmodium. However, we have briefly shown that two of the most commonly used ACTs do not change the composition of the gut microbiota in 
mice. As the gut microbiota has become an important area of clinical investigation, it is beneficial to know that ACT treatments do not seem to pose the same challenges as antibiotic treatments to gut homeostasis and host health. However, given the differences between mice and humans, it is critical to continue characterizing the role of the human gut microbiota during malaria infection and treatment to enhance the effort to defeat malaria as a global pathogen.

\section{Materials and Methods}

\section{Animals and Housing:}

6 week old female C57BL/6 mice were purchased from Envigo (Indianapolis, IN). All mice were housed in a specific pathogen-free facility and acclimatized for a minimum of 7 days before starting experiments. Animals were fed the NIH-31 diet (Modified Open Formula Mouse/Rat Irradiated Diet; Harlan 7913; Envigo, Indianapolis, IN) and provided autoclaved, non-acidified municipal water ad libitum. The mice were kept on a 12-hour light/dark cycle from $6 \mathrm{AM}$ to 6PM and 6PM to 6AM, respectively. All animal experiments were carried out at the University of Louisville in compliance with local and national regulations of laboratory animal welfare. Additionally, all animal care and use procedures were approved by the University of Louisville Institutional Animal Care and Use Committee. 


\section{Gut Microbiota Analysis:}

Mouse fecal pellets were collected at days $0,1,2,3,5,7,10,14$, and 21 p.t. and flash frozen in liquid nitrogen followed by storage at $-80^{\circ} \mathrm{C}$. DNA was extracted using the QIAamp PowerFecal DNA kit (QIAGEN, Germantown, MD) according to the manufacturer's instructions. DNA samples were then shipped overnight on ice to the Genome Technology Access Center at Washington University (GTAC, St. Louis, MO) for sequencing followed by analysis using MVRSION175. The OTU table received from GTAC was CSS normalized and analyzed within QIIME v1.9.1 to produce taxa plots and calculate alpha diversity and Bray-Curtis beta diversity 170 .

\section{Antimalarial Drug Treatments:}

For both $A L$ and $A A$, clinical dosing was used according to recommendations by the World Health Organization ${ }^{20}$. Artemether was given at 2 $\mathrm{mg} / \mathrm{kg}$ and lumefantrine at $12 \mathrm{mg} / \mathrm{kg}$, while artesunate was given at $4 \mathrm{mg} / \mathrm{kg}$ and amodiaquine at $10 \mathrm{mg} / \mathrm{kg}$. AL and AA were diluted in either $100 \%$ olive oil (Kroger, Cincinnati, OH, USA) or saline (Teknova, Hollister, CA, USA), respectively. Mice received $A L$ and AA by oral once daily for three days.

\section{Statistical Analysis:}


Statistical analyses were performed using GraphPad Prism 7 software

(GraphPad Software, La Jolla, CA, USA). Specific analyses are described in figure legends.

\section{Figures and Figure Legends}

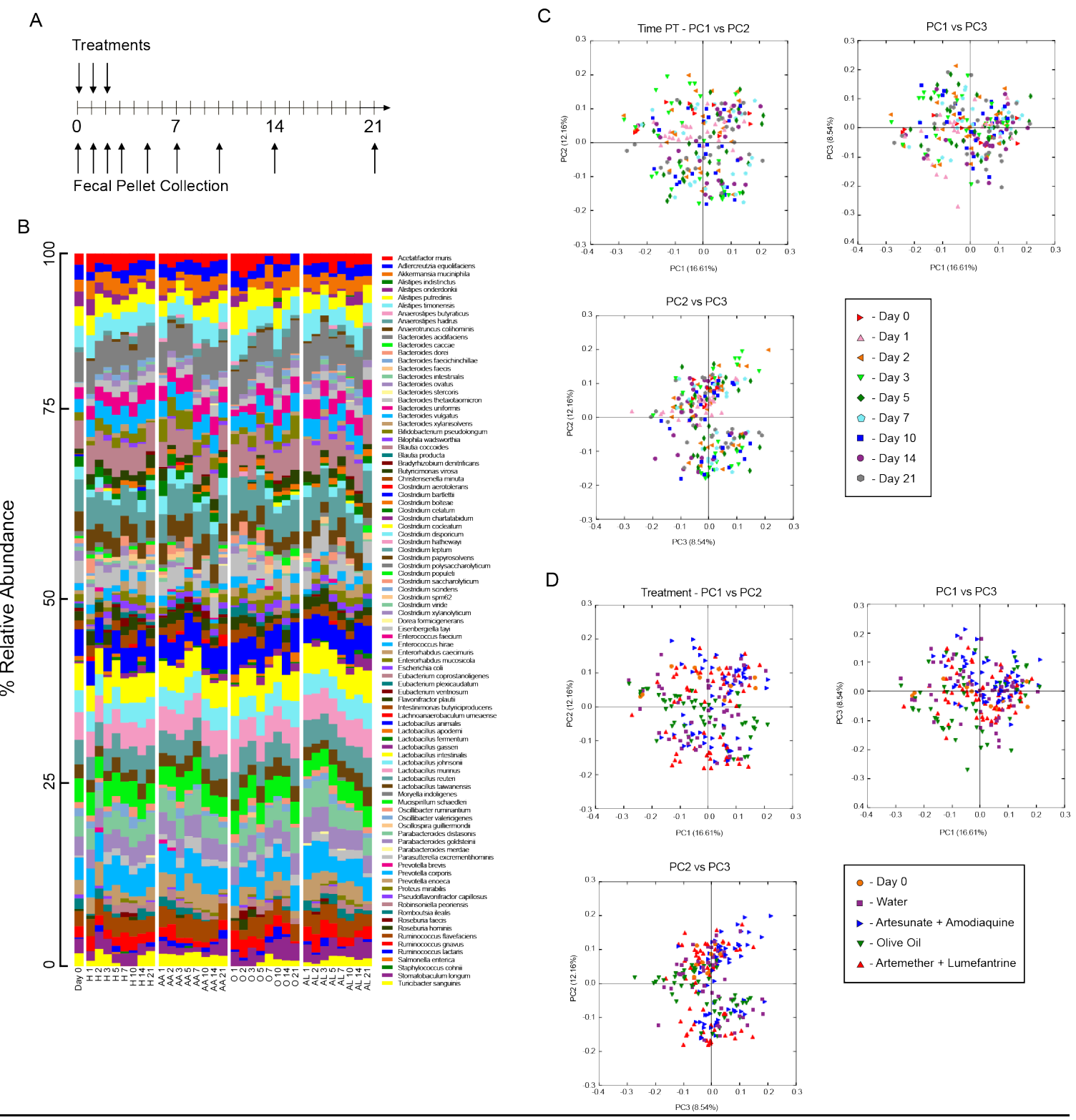


Figure 4-1. Relative bacterial abundances remain stable despite antimalarial treatments. A. Experimental design for antimalarial treatments and fecal pellet collections. B. Relative taxonomic abundances of bacterial species among different treatment groups. Time points are collapsed by average abundance and scaled to sum to 1. PCoA plots of Bray-Curtis distances between samples, with either C. time p.t. or D. treatment metadata distinguishing samples. Data are the cumulative results of 2 experiments (4 mice/group/experiment); untreated (day 0) data for each group is pooled. 


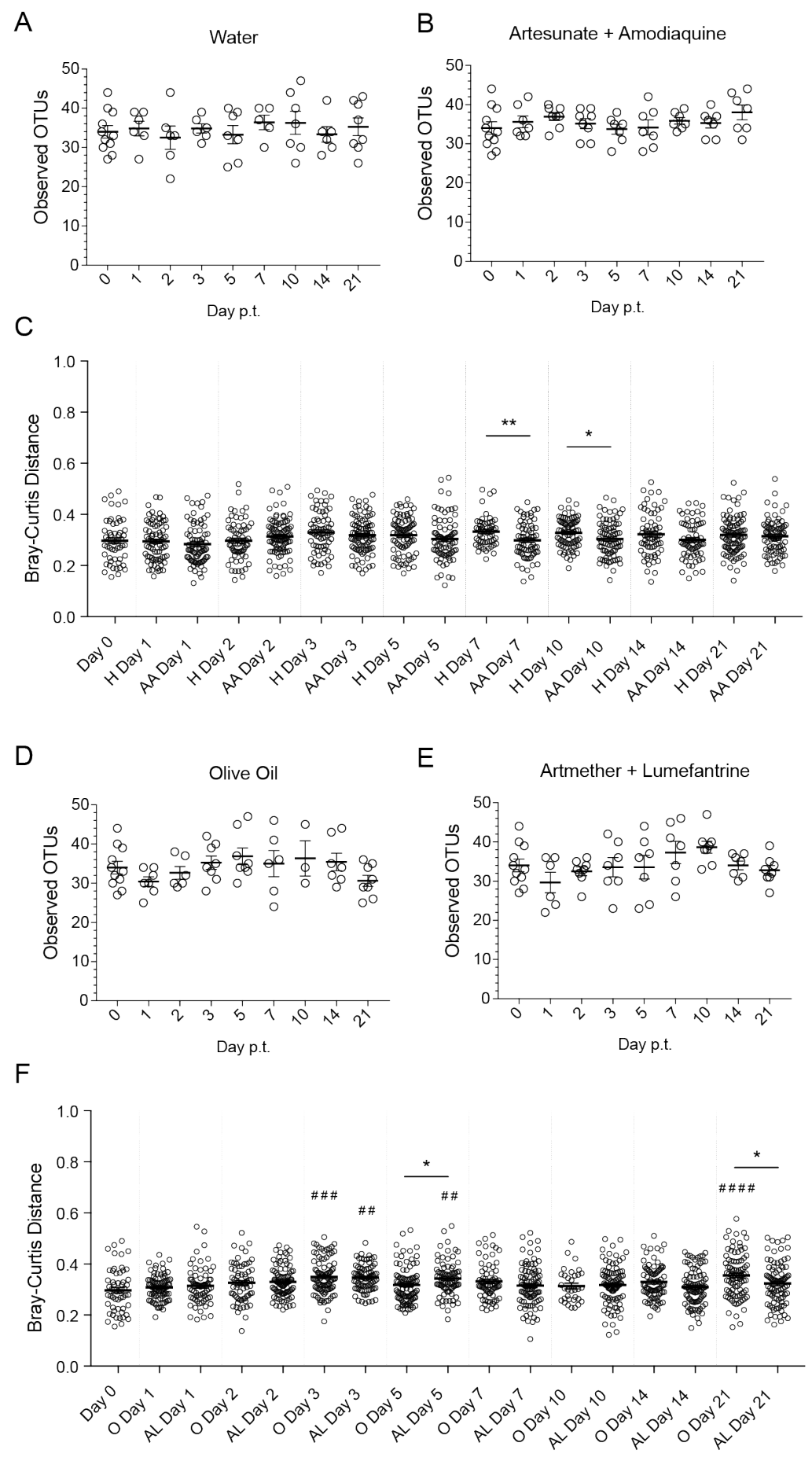

Figure 4-2. Antimalarial drug treatments cause minor changes to the diversity indices of the gut microbiota in mice. Alpha diversity of the gut microbiota as measured by observed OTUs in mice treated with $\mathbf{A}$. saline and $\mathbf{B}$. 
artesunate and amodiaquine. C. Bray-Curtis beta diversity for samples treated with either water or artesunate and amodiaquine. The distances compared are between the pooled day 0 samples and each treatment group at each time point followed by comparisons between treatments at each time point. D. Alpha diversity of samples from mice treated with olive oil and E. mice treated with artemether and lumefantrine. F. Bray-Curtis beta diversity comparisons for samples from mice treated with either olive oil or artemether and lumefantrine as in panel C. Data are the cumulative results of 2 experiments (4 mice/group/experiment); untreated (day 0) data for each group is pooled. Data in A-B and D-E were analyzed by one-way ANOVA with Tukey's multiple comparisons test; data in $\mathbf{C}$ and $\mathbf{F}$ were analyzed by one-way ANOVA with Dunnett's multiple comparisons test followed by a two-tailed t test for treatment comparisons at each time point. ${ }^{*}=$ comparison between same-day treatments; \# $=$ comparison between treatment day and day 0 p.t. 1 symbol $=p<0.05 ; 2$ symbols $=p<0.01 ; 3$ symbols $=p<0.001 ; 4$ symbols $=p<0.0001$. 


\section{CHAPTER 5}

\section{GENERAL DISCUSSION AND FUTURE DIRECTIONS}

It is critical to understand mechanisms of host resistance and Plasmodium disease pathogenesis. Identifying Plasmodium-host interactions that can be exploited for the production of antimalarial drugs or as vaccine candidates has always been a relevant strategy. However, since we have shown that the composition of the gut microbiota can modulate the severity of infection, the dimensionality and complexity of interactions increases to include Plasmodium, the host, and the microbiota. With this in mind, the search for malaria treatments is both easier and harder simultaneously. It is easier in that it may be possible to simply modulate the gut microbiota using a probiotic bacteria, prebiotic fiber, or metabolite to enhance the host response to malaria and inhibit the development of complicated malaria. On the other hand, antimalarial treatment design must also consider the microbiota and its effect not only on the severity of malaria, but also on other aspects of host physiology like metabolism or immunity. A treatment that affects 
the composition of the microbiota also has the potential to change the future resistance or susceptibility to severe malaria.

On a smaller scale, the complete mechanism for how the gut microbiota can regulate the severity of malaria is still unknown. Based on the observations made here, there are differences in host immunity during Py infection that correlate to differences in the gut microbiota; however, despite the fact that the compositions of both Tac and CR microbiotas become more similar over infection, these changes do not modulate severity of infection. With this in mind, one possible conclusion is that instead it is the differential function of the Tac and $\mathrm{CR}$ microbiotas that is responsible for the modulation of malaria severity. For example, Tac and CR mice receive the same diet with the same nutrients, but the cecal metabolite profiles are dramatically different during infection despite the decreasing dissimilarity between Tac and CR microbiotas. If the functional differences lead to the observed differences in severity of malaria, the products of the microbiota metabolism quite possibly are the key link between the microbiota and the host. However, this metabolite still needs to be identified.

One potential method to identify these metabolites that contribute to either resistant or susceptible phenotypes would be to isolate cecal or fecal cell-free supernatants from Tac or CR mice. These supernatants could then be given orally to identify the total contribution of the metabolites, or the supernatants could be treated beforehand to narrow down the class of metabolites that are active. For example, enzymatic treatments, heat treatments, and size 
fractionation would help identify whether the active metabolite or metabolites were a specific type of molecule, such as a nucleotide or peptide.

In germ-free mice, other options exist for identifying active microbes or metabolites in resistance or susceptibility. First, limited colonization of gnotobiotic mice with microbes of interest, such as Lactobacillus or Bifidobacteria species, would help determine the contribution of specific microbes or consortia of microbes to resistance or susceptibility. These microbes could have potential probiotic functionality for enhancing resistance to malaria. One caveat, however, is that the contribution of probiotics that we observed was moderate at best, and primarily dependent on antibiotic treatment beforehand. While this does not preclude probiotics as a potential treatment, it does highlight the resiliency of the established microbiota and the difficulty such future treatments may face. In a similar vein, maintaining germ-free mice but treating with metabolites of interest or postbiotics from the resistant microbiota may aid in identifying signals from the microbiota that confer the resistant or susceptible phenotypes. Also, as diet was shown to influence susceptibility to severe malaria, mice could also be given diets restricted in certain metabolites such as specific amino acids or flavonoids. These diet studies would complement experiments where mice are given an abundance of a specific metabolite.

On a different note, we showed that treatment with ACTs has very limited effects on the gut microbiota. However, one way to confirm that these changes do not affect susceptibility to Py infections is to treat with ACTs followed by Py 
infection; resistant and susceptible mice should have no changes in parasite burden or morbidity and mortality.

Within the context of human malaria, little work has been done to study how the gut microbiota modulates resistance or susceptibility to malaria. As discussed previously, different rodent models of malaria can recapitulate different aspects of human malaria; Py in particular can model the pathology and the initial immune response to malaria. Using mice with humanized microbiota would allow for initial characterization of how different human microbiota compositions modulate Py severity and the immune response to malaria. Comparisons could also be drawn between the baseline Tac or CR microbiota composition and function compared to the humanized microbiota. Prebiotic, probiotic, or antibiotic interventions could be used to modify the humanized microbiota to resemble the Tac microbiota to enhance resistance to Py. It would also be beneficial to perform large-scale sequencing efforts to identify microbiota profiles in humans that correlate with protection from malaria, as well as contributing factors like diet.

Overall, this work constitutes extensive characterization of how gut homeostasis affects and is affected by mild or severe Plasmodium infection, as well as a brief analysis of how clinical ACTs affect the composition of the gut microbiota. As the findings associated with the gut microbiota and its interactions with malaria are recent, it is hoped that these observations assist in future 
research into the mechanisms of the modulation of severe malaria by the microbiota. 


\section{REFERENCES}

1 Organization, W. H. World Malaria Report 2017, $<$ http://www.who.int/malaria/publications/world-malaria-report-2017/en/> (2017).

2 Marsh, K. et al. Indicators of life-threatening malaria in African children. The New England journal of medicine 332, 1399-1404, doi:10.1056/nejm199505253322102 (1995).

3 Crompton, P. D. et al. Malaria immunity in man and mosquito: insights into unsolved mysteries of a deadly infectious disease. Annual review of immunology 32, 157-187, doi:10.1146/annurev-immunol-032713-120220 (2014).

4 Pumpuni, C. B., Mendis, C. \& Beier, J. C. Plasmodium yoelii sporozoite infectivity varies as a function of sporozoite loads in Anopheles stephensi mosquitoes. The Journal of parasitology 83, 652-655 (1997).

5 Sturm, A. et al. Manipulation of host hepatocytes by the malaria parasite for delivery into liver sinusoids. Science (New York, N.Y.) 313, 1287-1290, doi:10.1126/science.1129720 (2006).

6 Stephens, R., Culleton, R. L. \& Lamb, T. J. The contribution of Plasmodium chabaudi to our understanding of malaria. Trends in parasitology 28, 73-82, doi:10.1016/j.pt.2011.10.006 (2012).

7 Gaur, D., Mayer, D. C. \& Miller, L. H. Parasite ligand-host receptor interactions during invasion of erythrocytes by Plasmodium merozoites. International journal for parasitology 34, 1413-1429, doi:10.1016/j.ijpara.2004.10.010 (2004).

8 Paul, A. S., Egan, E. S. \& Duraisingh, M. T. Host-parasite interactions that guide red blood cell invasion by malaria parasites. Current opinion in hematology 22, 220-226, doi:10.1097/moh.0000000000000135 (2015).

9 Loy, D. E. et al. Out of Africa: origins and evolution of the human malaria parasites Plasmodium falciparum and Plasmodium vivax. International journal for parasitology 47, 87-97, doi:10.1016/j.jpara.2016.05.008 (2017).

10 Eikenberry, S. E. \& Gumel, A. B. Mathematical modeling of climate change and malaria transmission dynamics: a historical review. Journal of mathematical biology 77, 857-933, doi:10.1007/s00285-018-1229-7 (2018). 
11 Cowman, A. F., Tonkin, C. J., Tham, W. H. \& Duraisingh, M. T. The Molecular Basis of Erythrocyte Invasion by Malaria Parasites. Cell host \& microbe 22, 232-245, doi:10.1016/j.chom.2017.07.003 (2017).

12 Achtman, A. H., Stephens, R., Cadman, E. T., Harrison, V. \& Langhorne, $J$. Malaria-specific antibody responses and parasite persistence after infection of mice with Plasmodium chabaudi chabaudi. Parasite immunology 29, 435-444, doi:10.1111/j.1365-3024.2007.00960.x (2007).

13 Taylor-Robinson, A. W. Regulation of immunity to Plasmodium: implications from mouse models for blood stage malaria vaccine design. Experimental parasitology 126, 406-414, doi:10.1016/j.exppara.2010.01.028 (2010).

14 Mikolajczak, S. A. et al. Plasmodium vivax liver stage development and hypnozoite persistence in human liver-chimeric mice. Cell host \& microbe 17, 526-535, doi:10.1016/j.chom.2015.02.011 (2015).

15 White, N. J. Determinants of relapse periodicity in Plasmodium vivax malaria. Malaria journal 10, 297, doi:10.1186/1475-2875-10-297 (2011).

16 Belnoue, E. et al. Control of pathogenic CD8+ T cell migration to the brain by IFN-gamma during experimental cerebral malaria. Parasite immunology 30, 544-553, doi:10.1111/j.1365-3024.2008.01053.x (2008).

17 Stevenson, M. M., Gros, P., Olivier, M., Fortin, A. \& Serghides, L. Cerebral malaria: human versus mouse studies. Trends in parasitology 26, 274275, doi:10.1016/j.pt.2010.03.008 (2010).

$18 \mathrm{Li}, \mathrm{C} . \&$ Langhorne, J. Tumor necrosis factor alpha p55 receptor is important for development of memory responses to blood-stage malaria infection. Infection and immunity 68, 5724-5730 (2000).

19 Sarfo, B. Y. et al. Plasmodium yoelii 17XL infection up-regulates RANTES, CCR1, CCR3 and CCR5 expression, and induces ultrastructural changes in the cerebellum. Malaria journal 4, 63, doi:10.1186/1475-2875-4-63 (2005).

20 Organization, W. H. Guidelines for the treatment of malaria. Third edition. (World Health Organization, 2015).

21 Thwing, J., Eisele, T. P. \& Steketee, R. W. Protective efficacy of malaria case management and intermittent preventive treatment for preventing malaria mortality in children: a systematic review for the Lives Saved Tool. BMC public health 11 Suppl 3, S14, doi:10.1186/1471-2458-11-s3-s14 (2011).

22 Clark, I. A., Alleva, L. M., Budd, A. C. \& Cowden, W. B. Understanding the role of inflammatory cytokines in malaria and related diseases. Travel medicine and infectious disease 6, 67-81, doi:10.1016/j.tmaid.2007.07.002 (2008).

23 Shikani, H. J. et al. Cerebral malaria: we have come a long way. The American journal of pathology 181, 1484-1492, doi:10.1016/j.ajpath.2012.08.010 (2012). 
24 Bauer, P. R., Van Der Heyde, H. C., Sun, G., Specian, R. D. \& Granger, D. N. Regulation of endothelial cell adhesion molecule expression in an experimental model of cerebral malaria. Microcirculation (New York, N.Y. : 1994) 9, 463-470, doi:10.1038/sj.mn.7800159 (2002).

25 Prasad, R. N. \& Virk, K. J. Malaria as a cause of diarrhoea--a review. Papua and New Guinea medical journal 36, 337-341 (1993).

26 De Souza, J. B., Williamson, K. H., Otani, T. \& Playfair, J. H. Early gamma interferon responses in lethal and nonlethal murine blood-stage malaria. Infection and immunity 65, 1593-1598 (1997).

27 Miller, J. L., Sack, B. K., Baldwin, M., Vaughan, A. M. \& Kappe, S. H. Interferon-mediated innate immune responses against malaria parasite liver stages. Cell reports 7, 436-447, doi:10.1016/j.celrep.2014.03.018 (2014).

28 Stevenson, M. M. \& Riley, E. M. Innate immunity to malaria. Nature reviews. Immunology 4, 169-180, doi:10.1038/nri1311 (2004).

29 Inoue, S., Niikura, M., Mineo, S. \& Kobayashi, F. Roles of IFN-gamma and gammadelta T Cells in Protective Immunity Against Blood-Stage Malaria. Frontiers in Immunology 4, 258, doi:10.3389/fimmu.2013.00258 (2013).

30 Roark, C. L., Simonian, P. L., Fontenot, A. P., Born, W. K. \& O'Brien, R. L. gammadelta T cells: an important source of IL-17. Current opinion in immunology 20, 353-357, doi:10.1016/j.coi.2008.03.006 (2008).

31 Ribot, J. C., Ribeiro, S. T., Correia, D. V., Sousa, A. E. \& Silva-Santos, B. Human gammadelta thymocytes are functionally immature and differentiate into cytotoxic type 1 effector T cells upon IL-2/IL-15 signaling. Journal of immunology (Baltimore, Md. : 1950) 192, 2237-2243, doi:10.4049/jimmunol.1303119 (2014).

32 Rezende, R. M. et al. gammadelta T cells control humoral immune response by inducing $\mathrm{T}$ follicular helper cell differentiation. Nature communications 9, 3151, doi:10.1038/s41467-018-05487-9 (2018).

33 Ribot, J. C. et al. Cutting edge: adaptive versus innate receptor signals selectively control the pool sizes of murine IFN-gamma- or IL-17producing gammadelta T cells upon infection. Journal of immunology (Baltimore, Md. : 1950) 185, 6421-6425, doi:10.4049/jimmunol.1002283 (2010).

34 Pan, T. et al. IL17-Producing gammadelta T Cells May Enhance Humoral Immunity during Pulmonary Pseudomonas aeruginosa Infection in Mice. Front Cell Infect Microbiol 6, 170, doi:10.3389/fcimb.2016.00170 (2016).

35 Sampaio, N. G., Eriksson, E. M. \& Schofield, L. Plasmodium falciparum PfEMP1 Modulates Monocyte/Macrophage Transcription Factor Activation and Cytokine and Chemokine Responses. Infection and immunity 86, doi:10.1128/iai.00447-17 (2018).

36 Parroche, P. et al. Malaria hemozoin is immunologically inert but radically enhances innate responses by presenting malaria DNA to Toll-like receptor 9. Proceedings of the National Academy of Sciences of the 
United States of America 104, 1919-1924, doi:10.1073/pnas.0608745104 (2007).

37 Mooney, J. P. et al. Inflammation-associated alterations to the intestinal microbiota reduce colonization resistance against non-typhoidal

Salmonella during concurrent malaria parasite infection. Scientific reports 5, 14603, doi:10.1038/srep14603 (2015).

38 Taniguchi, T. et al. Plasmodium berghei ANKA causes intestinal malaria associated with dysbiosis. Scientific reports 5, 15699, doi:10.1038/srep15699 (2015).

39 Vallejo, A. F. et al. Malaria systems immunology: Plasmodium vivax induces tolerance during primary infection through dysregulation of neutrophils and dendritic cells. The Journal of infection, doi:10.1016/j.jinf.2018.09.005 (2018).

40 Cockburn, I. A. \& Zavala, F. Dendritic cell function and antigen presentation in malaria. Current opinion in immunology 40, 1-6, doi:10.1016/j.coi.2016.01.010 (2016).

41 Wykes, M., Keighley, C., Pinzon-Charry, A. \& Good, M. F. Dendritic cell biology during malaria. Cellular microbiology 9, 300-305, doi:10.1111/j.1462-5822.2006.00865.x (2007).

42 Urban, B. C. et al. Plasmodium falciparum-infected erythrocytes modulate the maturation of dendritic cells. Nature 400, 73-77, doi:10.1038/21900 (1999).

43 Perry, J. A., Rush, A., Wilson, R. J., Olver, C. S. \& Avery, A. C. Dendritic cells from malaria-infected mice are fully functional APC. Journal of immunology (Baltimore, Md. : 1950) 172, 475-482 (2004).

44 Perez-Mazliah, D. et al. Disruption of IL-21 signaling affects T cell-B cell interactions and abrogates protective humoral immunity to malaria. PLOS pathogens 11, e1004715, doi:10.1371/journal.ppat.1004715 (2015).

45 Osier, F. H. et al. Breadth and magnitude of antibody responses to multiple Plasmodium falciparum merozoite antigens are associated with protection from clinical malaria. Infection and immunity 76, 2240-2248, doi:10.1128/iai.01585-07 (2008).

46 Hansen, D. S., Obeng-Adjei, N., Ly, A., loannidis, L. J. \& Crompton, P. D. Emerging concepts in $\mathrm{T}$ follicular helper cell responses to malaria. International journal for parasitology 47, 105-110, doi:10.1016/j.ijpara.2016.09.004 (2017).

47 Beeson, J. G. et al. Merozoite surface proteins in red blood cell invasion, immunity and vaccines against malaria. FEMS microbiology reviews $\mathbf{4 0}$, 343-372, doi:10.1093/femsre/fuw001 (2016).

48 Teo, A., Feng, G., Brown, G. V., Beeson, J. G. \& Rogerson, S. J. Functional Antibodies and Protection against Blood-stage Malaria. Trends in parasitology 32, 887-898, doi:10.1016/j.pt.2016.07.003 (2016). 
49 Foquet, L. et al. Molecular detection and quantification of Plasmodium falciparum-infected human hepatocytes in chimeric immune-deficient mice. Malaria journal 12, 430, doi:10.1186/1475-2875-12-430 (2013).

50 Tran, T. M. et al. An intensive longitudinal cohort study of Malian children and adults reveals no evidence of acquired immunity to Plasmodium falciparum infection. Clinical infectious diseases : an official publication of the Infectious Diseases Society of America 57, 40-47, doi:10.1093/cid/cit174 (2013).

51 Butler, N. S. et al. Therapeutic blockade of PD-L1 and LAG-3 rapidly clears established blood-stage Plasmodium infection. Nat Immunol 13, 188-195, doi:10.1038/ni.2180 (2011).

52 Illingworth, J. et al. Chronic exposure to Plasmodium falciparum is associated with phenotypic evidence of $B$ and $T$ cell exhaustion. Journal of immunology (Baltimore, Md. : 1950) 190, 1038-1047, doi:10.4049/jimmunol.1202438 (2013).

53 Ryg-Cornejo, V. et al. Severe Malaria Infections Impair Germinal Center Responses by Inhibiting T Follicular Helper Cell Differentiation. Cell reports 14, 68-81, doi:10.1016/j.celrep.2015.12.006 (2016).

54 Combrinck, J. M. et al. Insights into the Role of Heme in the Mechanism of Action of Antimalarials. ACS chemical biology 8, 133-137, doi:10.1021/cb300454t (2013).

55 Bridgford, J. L. et al. Artemisinin kills malaria parasites by damaging proteins and inhibiting the proteasome. Nature communications 9 , 3801, doi:10.1038/s41467-018-06221-1 (2018).

56 Gunjan, S. et al. Artemisinin Derivatives and Synthetic Trioxane Trigger Apoptotic Cell Death in Asexual Stages of Plasmodium. Front Cell Infect Microbiol 8, 256, doi:10.3389/fcimb.2018.00256 (2018).

57 Goswami, S. et al. Anti-Helicobacter pylori Potential of Artemisinin and Its Derivatives. Antimicrobial Agents and Chemotherapy 56, 4594-4607, doi:10.1128/aac.00407-12 (2012).

58 Sisto, F. et al. In vitro activity of artemisone and artemisinin derivatives against extracellular and intracellular Helicobacter pylori. International Journal of Antimicrobial Agents 48, 101-105, doi:https://doi.org/10.1016/j.ijantimicag.2016.03.018 (2016).

59 Caballero, S. et al. Cooperating commensals restore colonization resistance to vancomycin- resistant Enterococcus faecium. Cell host \& microbe 21, 592-602.e594, doi:10.1016/j.chom.2017.04.002 (2017).

60 Cox, L. M. et al. Altering the intestinal microbiota during a critical developmental window has lasting metabolic consequences. Cell 158, 705-721, doi:10.1016/j.cell.2014.05.052 (2014).

61 Johnson, C. C. et al. Antibiotic exposure in early infancy and risk for childhood atopy. The Journal of allergy and clinical immunology 115, 1218-1224, doi:10.1016/j.jaci.2005.04.020 (2005). 
62 Buffie, C. G. et al. Profound alterations of intestinal microbiota following a single dose of clindamycin results in sustained susceptibility to Clostridium difficile-induced colitis. Infection and immunity 80, 62-73, doi:10.1128/iai.05496-11 (2012).

63 David, L. A. et al. Diet rapidly and reproducibly alters the human gut microbiome. Nature 505, 559-563, doi:10.1038/nature12820 (2014).

64 Wilck, N. et al. Salt-responsive gut commensal modulates TH17 axis and disease. Nature 551, 585-589, doi:10.1038/nature24628 (2017).

65 Koh, A., De Vadder, F., Kovatcheva-Datchary, P. \& Backhed, F. From Dietary Fiber to Host Physiology: Short-Chain Fatty Acids as Key Bacterial Metabolites. Cell 165, 1332-1345, doi:10.1016/j.cell.2016.05.041 (2016).

66 Denny, J. E., Powell, W. L. \& Schmidt, N. W. Local and Long-Distance Calling: Conversations between the Gut Microbiota and Intra- and ExtraGastrointestinal Tract Infections. Frontiers in Cellular and Infection Microbiology 6, doi:10.3389/fcimb.2016.00041 (2016).

67 Leber, A. et al. Systems Modeling of Interactions between Mucosal Immunity and the Gut Microbiome during Clostridium difficile Infection. PloS one 10, e0134849, doi:10.1371/journal.pone.0134849 (2015).

68 Hirsch, B. E. et al. Effectiveness of fecal-derived microbiota transfer using orally administered capsules for recurrent Clostridium difficile infection. BMC infectious diseases 15, 191, doi:10.1186/s12879-015-0930-z (2015).

69 Weingarden, A. R. et al. Microbiota transplantation restores normal fecal bile acid composition in recurrent Clostridium difficile infection. American journal of physiology. Gastrointestinal and liver physiology 306, G310-319, doi:10.1152/ajpgi.00282.2013 (2014).

70 Buffie, C. G. et al. Precision microbiome reconstitution restores bile acid mediated resistance to Clostridium difficile. Nature 517, 205-208, doi:10.1038/nature13828 (2015).

71 Ichinohe, T. et al. Microbiota regulates immune defense against respiratory tract influenza A virus infection. Proceedings of the National Academy of Sciences of the United States of America 108, 5354-5359, doi:10.1073/pnas.1019378108 (2011).

72 Abt, M. C. et al. Commensal bacteria calibrate the activation threshold of innate antiviral immunity. Immunity $37,158-170$, doi:10.1016/j.immuni.2012.04.011 (2012).

73 Wang, J. et al. Respiratory influenza virus infection induces intestinal immune injury via microbiota-mediated Th17 cell-dependent inflammation. The Journal of experimental medicine 211, 2397-2410, doi:10.1084/jem.20140625 (2014).

74 Yilmaz, B. et al. Gut microbiota elicits a protective immune response against malaria transmission. Cell 159, 1277-1289, doi:10.1016/j.cell.2014.10.053 (2014). 
75 Murray, C. J. et al. Global malaria mortality between 1980 and 2010: a systematic analysis. Lancet (London, England) 379, 413-431, doi:10.1016/s0140-6736(12)60034-8 (2012).

76 Dondorp, A. M. et al. Estimation of the total parasite biomass in acute falciparum malaria from plasma PfHRP2. PLoS medicine 2, e204, doi:10.1371/journal.pmed.0020204 (2005).

77 Hanson, J. et al. Relative contributions of macrovascular and microvascular dysfunction to disease severity in falciparum malaria. The Journal of infectious diseases 206, 571-579, doi:10.1093/infdis/jis400 (2012).

78 Honda, K. \& Littman, D. R. The microbiome in infectious disease and inflammation. Annual review of immunology 30, 759-795, doi:10.1146/annurev-immunol-020711-074937 (2012).

79 Turnbaugh, P. J. et al. An obesity-associated gut microbiome with increased capacity for energy harvest. Nature 444, 1027-1131, doi:http://www.nature.com/nature/journal/v444/n7122/suppinfo/nature0541 4_S1.html (2006).

80 Wen, L. et al. Innate immunity and intestinal microbiota in the development of Type 1 diabetes. Nature 455, 1109-1113, doi:10.1038/nature07336 (2008).

81 Hsiao, E. Y. et al. Microbiota modulate behavioral and physiological abnormalities associated with neurodevelopmental disorders. Cell 155, 1451-1463, doi:10.1016/j.cell.2013.11.024 (2013).

82 Joffe, T. H. \& Simpson, N. A. Cesarean section and risk of asthma. The role of intrapartum antibiotics: a missing piece? The Journal of pediatrics 154, 154, doi:10.1016/j.jpeds.2008.08.039 (2009).

83 Fujimura, K. E. et al. House dust exposure mediates gut microbiome Lactobacillus enrichment and airway immune defense against allergens and virus infection. Proceedings of the National Academy of Sciences of the United States of America 111, 805-810, doi:10.1073/pnas.1310750111 (2014).

84 Manichanh, C. et al. Reduced diversity of faecal microbiota in Crohn's disease revealed by a metagenomic approach. Gut 55, 205-211, doi:10.1136/gut.2005.073817 (2006).

85 Frank, D. N. et al. Molecular-phylogenetic characterization of microbial community imbalances in human inflammatory bowel diseases.

Proceedings of the National Academy of Sciences of the United States of America 104, 13780-13785, doi:10.1073/pnas.0706625104 (2007).

86 Peterson, D. A., Frank, D. N., Pace, N. R. \& Gordon, J. I. Metagenomic approaches for defining the pathogenesis of inflammatory bowel diseases. Cell host \& microbe 3, 417-427, doi:10.1016/j.chom.2008.05.001 (2008).

87 Nicholson, J. K. et al. Host-gut microbiota metabolic interactions. Science (New York, N.Y.) 336, 1262-1267, doi:10.1126/science.1223813 (2012). 
88 Kamada, N., Chen, G. Y., Inohara, N. \& Nunez, G. Control of pathogens and pathobionts by the gut microbiota. Nat Immunol 14, 685-690, doi:10.1038/ni.2608 (2013).

89 Benson, A., Pifer, R., Behrendt, C. L., Hooper, L. V. \& Yarovinsky, F. Gut commensal bacteria direct a protective immune response against Toxoplasma gondii. Cell host \& microbe 6, 187-196, doi:10.1016/j.chom.2009.06.005 (2009).

90 Naik, S. et al. Compartmentalized control of skin immunity by resident commensals. Science (New York, N.Y.) 337, 1115-1119, doi:10.1126/science.1225152 (2012).

91 Yooseph, S. et al. Stool microbiota composition is associated with the prospective risk of Plasmodium falciparum infection. BMC genomics 16, 631, doi:10.1186/s12864-015-1819-3 (2015).

92 Ivanov, II et al. Induction of intestinal Th17 cells by segmented filamentous bacteria. Cell 139, 485-498, doi:10.1016/j.cell.2009.09.033 (2009).

93 Ivanov, II et al. Specific microbiota direct the differentiation of IL-17producing T-helper cells in the mucosa of the small intestine. Cell host \& microbe 4, 337-349, doi:10.1016/j.chom.2008.09.009 (2008).

94 Turnbaugh, P. J. et al. The effect of diet on the human gut microbiome: a metagenomic analysis in humanized gnotobiotic mice. Sci Transl Med 1, 6ra14, doi:10.1126/scitranslmed.3000322 (2009).

95 Carmody, R. N. et al. Diet dominates host genotype in shaping the murine gut microbiota. Cell host \& microbe 17, 72-84, doi:10.1016/j.chom.2014.11.010 (2015).

96 Perez-Enciso, M. \& Tenenhaus, M. Prediction of clinical outcome with microarray data: a partial least squares discriminant analysis (PLS-DA) approach. Human genetics 112, 581-592, doi:10.1007/s00439-003-0921-9 (2003).

97 Segata, N. et al. Metagenomic biomarker discovery and explanation. Genome biology 12, R60, doi:10.1186/gb-2011-12-6-r60 (2011).

98 Cox, M. J. et al. Lactobacillus casei abundance is associated with profound shifts in the infant gut microbiome. PloS one 5, e8745, doi:10.1371/journal.pone.0008745 (2010).

99 McNulty, N. P. et al. The impact of a consortium of fermented milk strains on the gut microbiome of gnotobiotic mice and monozygotic twins. Sci Transl Med 3, 106ra106, doi:10.1126/scitranslmed.3002701 (2011).

100 Azambuja, P., Garcia, E. S. \& Ratcliffe, N. A. Gut microbiota and parasite transmission by insect vectors. Trends in parasitology 21, 568-572, doi:10.1016/j.pt.2005.09.011 (2005).

101 Dong, Y., Manfredini, F. \& Dimopoulos, G. Implication of the mosquito midgut microbiota in the defense against malaria parasites. PLoS pathogens 5, e1000423, doi:10.1371/journal.ppat.1000423 (2009). 
102 Cirimotich, C. M. et al. Natural microbe-mediated refractoriness to Plasmodium infection in Anopheles gambiae. Science (New York, N.Y.) 332, 855-858, doi:10.1126/science.1201618 (2011).

103 Boissiere, A. et al. Midgut microbiota of the malaria mosquito vector Anopheles gambiae and interactions with Plasmodium falciparum infection. PLoS pathogens 8, e1002742, doi:10.1371/journal.ppat.1002742 (2012).

104 Bahia, A. C. et al. Exploring Anopheles gut bacteria for Plasmodium blocking activity. Environmental microbiology 16, 2980-2994, doi:10.1111/1462-2920.12381 (2014).

105 Verhulst, N. O. et al. Cultured skin microbiota attracts malaria mosquitoes. Malaria journal 8, 302, doi:10.1186/1475-2875-8-302 (2009).

106 Verhulst, N. O. et al. Composition of human skin microbiota affects attractiveness to malaria mosquitoes. PloS one 6, e28991, doi:10.1371/journal.pone.0028991 (2011).

107 Kau, A. L., Ahern, P. P., Griffin, N. W., Goodman, A. L. \& Gordon, J. I. Human nutrition, the gut microbiome and the immune system. Nature 474, 327-336, doi:10.1038/nature10213 (2011).

108 Brestoff, J. R. \& Artis, D. Commensal bacteria at the interface of host metabolism and the immune system. Nat Immunol 14, 676-684, doi:10.1038/ni.2640 (2013).

109 De Filippo, C. et al. Impact of diet in shaping gut microbiota revealed by a comparative study in children from Europe and rural Africa. Proceedings of the National Academy of Sciences of the United States of America 107, 14691-14696, doi:10.1073/pnas.1005963107 (2010).

110 Schloss, P. D. et al. Introducing mothur: open-source, platformindependent, community-supported software for describing and comparing microbial communities. Applied and environmental microbiology 75, 75377541, doi:10.1128/aem.01541-09 (2009).

111 Clarke, K. \& Gorley, R. PRIMER v6: user manual/tutorial. (2006).

$112 \mathrm{Lu}, \mathrm{W}$. et al. Metabolomic analysis via reversed-phase ion-pairing liquid chromatography coupled to a stand alone orbitrap mass spectrometer. Analytical chemistry 82, 3212-3221, doi:10.1021/ac902837x (2010).

113 Martens, L. et al. mzML--a community standard for mass spectrometry data. Molecular \& cellular proteomics : MCP 10, R110.000133, doi:10.1074/mcp.R110.000133 (2011).

114 Chambers, M. C. et al. A cross-platform toolkit for mass spectrometry and proteomics. Nature biotechnology 30, 918-920, doi:10.1038/nbt.2377 (2012).

115 Clasquin, M. F., Melamud, E. \& Rabinowitz, J. D. LC-MS data processing with MAVEN: a metabolomic analysis and visualization engine. Current protocols in bioinformatics Chapter 14, Unit14.11, doi:10.1002/0471250953.bi1411s37 (2012). 
116 Melamud, E., Vastag, L. \& Rabinowitz, J. D. Metabolomic analysis and visualization engine for LC-MS data. Analytical chemistry 82, 9818-9826, doi:10.1021/ac1021166 (2010).

117 de Hoon, M. J., Imoto, S., Nolan, J. \& Miyano, S. Open source clustering software. Bioinformatics (Oxford, England) 20, 1453-1454, doi:10.1093/bioinformatics/bth078 (2004).

118 Saldanha, A. J. Java Treeview--extensible visualization of microarray data. Bioinformatics (Oxford, England) 20, 3246-3248, doi:10.1093/bioinformatics/bth349 (2004).

119 Wickham, H. ggplot2: elegant graphics for data analysis. (Springer, 2016).

120 Vu, V. Q. ggbiplot: A ggplot2 based biplot. (2011).

121 Dejean, S. et al. mixOmics: omics data integration project. (2014).

122 Matthews, J. N., Altman, D. G., Campbell, M. J. \& Royston, P. Analysis of serial measurements in medical research. BMJ (Clinical research ed.) 300, 230-235 (1990).

123 Mendez, F., Munoz, A. \& Plowe, C. V. Use of area under the curve to characterize transmission potential after antimalarial treatment. The American Journal of Tropical Medicine and Hygiene 75, 640-644 (2006).

124 Dondorp, A. M. et al. Artemisinin Resistance in Plasmodium falciparum Malaria. New England Journal of Medicine 361, 455-467, doi:10.1056/NEJMoa0808859 (2009).

125 Amato, R. et al. Genetic markers associated with dihydroartemisininpiperaquine failure in Plasmodium falciparum malaria in Cambodia: a genotype-phenotype association study. The Lancet. Infectious diseases 17, 164-173, doi:10.1016/s1473-3099(16)30409-1 (2017).

126 Michalakis, Y. \& Renaud, F. Malaria: Evolution in vector control. Nature 462, 298-300, doi:10.1038/462298a (2009).

127 Sender, R., Fuchs, S. \& Milo, R. Are We Really Vastly Outnumbered? Revisiting the Ratio of Bacterial to Host Cells in Humans. Cell 164, 337340, doi:10.1016/j.cell.2016.01.013 (2016).

128 Grice, E. A. et al. A diversity profile of the human skin microbiota. Genome Research 18, 1043-1050, doi:10.1101/gr.075549.107 (2008).

129 Costello, E. K. et al. Bacterial Community Variation in Human Body Habitats Across Space and Time. Science (New York, N.Y.) 326, 1694 (2009).

130 Hilty, M. et al. Disordered microbial communities in asthmatic airways. PloS one 5, e8578, doi:10.1371/journal.pone.0008578 (2010).

131 Charlson, E. S. et al. Topographical continuity of bacterial populations in the healthy human respiratory tract. American journal of respiratory and critical care medicine 184, 957-963, doi:10.1164/rccm.201104-0655OC (2011).

132 Huang, Y. J. et al. Airway microbiota and bronchial hyperresponsiveness in patients with suboptimally controlled asthma. The Journal of allergy and 
clinical immunology 127, 372-381.e371-373,

doi:10.1016/j.jaci.2010.10.048 (2011).

133 Dash, S., Clarke, G., Berk, M. \& Jacka, F. N. The gut microbiome and diet in psychiatry: focus on depression. Current opinion in psychiatry 28, 1-6, doi:10.1097/yco.0000000000000117 (2015).

134 Fagundes, C. T. et al. Transient TLR activation restores inflammatory response and ability to control pulmonary bacterial infection in germfree mice. Journal of immunology (Baltimore, Md. : 1950) 188, 1411-1420, doi:10.4049/jimmunol.1101682 (2012).

135 Clarke, T. B. Early innate immunity to bacterial infection in the lung is regulated systemically by the commensal microbiota via nod-like receptor ligands. Infection and immunity 82, 4596-4606, doi:10.1128/iai.02212-14 (2014).

136 Villarino, N. F. et al. Composition of the gut microbiota modulates the severity of malaria. Proceedings of the National Academy of Sciences of the United States of America 113, 2235-2240, doi:10.1073/pnas.1504887113 (2016).

137 Atarashi, K. et al. Induction of colonic regulatory T cells by indigenous Clostridium species. Science (New York, N. Y.) 331, 337-341, doi:10.1126/science.1198469 (2011).

138 Chung, H. et al. Gut immune maturation depends on colonization with a host-specific microbiota. Cell 149, 1578-1593, doi:10.1016/j.cell.2012.04.037 (2012).

139 Geuking, M. B. et al. Intestinal bacterial colonization induces mutualistic regulatory T cell responses. Immunity 34, 794-806, doi:10.1016/j.immuni.2011.03.021 (2011).

140 Cai, X. et al. Dietary nucleotides protect against alcoholic liver injury by attenuating inflammation and regulating gut microbiota in rats. Food \& function 7, 2898-2908, doi:10.1039/c5fo01580d (2016).

141 Zelante, T. et al. Tryptophan catabolites from microbiota engage aryl hydrocarbon receptor and balance mucosal reactivity via interleukin-22. Immunity 39, 372-385, doi:10.1016/j.immuni.2013.08.003 (2013).

142 Wagage, S. et al. The aryl hydrocarbon receptor promotes IL-10 production by NK cells. Journal of immunology (Baltimore, Md. : 1950) 192, 1661-1670, doi:10.4049/jimmunol.1300497 (2014).

143 Mimche, P. N. et al. The receptor tyrosine kinase EphB2 promotes hepatic fibrosis in mice. Hepatology (Baltimore, Md.) 62, 900-914, doi:10.1002/hep.27792 (2015).

144 Brown, W. R. \& Kloppel, T. M. The role of the liver in translocation of IgA into the gastrointestinal tract. Immunological investigations 18, 269-285 (1989).

145 Paik, Y.-H. et al. Toll-Like receptor 4 mediates inflammatory signaling by bacterial lipopolysaccharide in human hepatic stellate cells. Hepatology 
(Baltimore, Md.) 37, 1043-1055,

doi:https://doi.org/10.1053/jhep.2003.50182 (2003).

146 Balmer, M. L. et al. The Liver May Act as a Firewall Mediating Mutualism Between the Host and Its Gut Commensal Microbiota. Science Translational Medicine 6, 237 ra266 (2014).

$147 \mathrm{Li}$, F. et al. The microbiota maintain homeostasis of liver-resident gammadeltaT-17 cells in a lipid antigen/CD1d-dependent manner. Nature communications 7, 13839, doi:10.1038/ncomms13839 (2017).

$148 \mathrm{Kim}, \mathrm{l}$. et al. Differential regulation of bile acid homeostasis by the farnesoid $X$ receptor in liver and intestine. Journal of lipid research 48, 2664-2672, doi:10.1194/jlr.M700330-JLR200 (2007).

149 Kawamata, Y. et al. A G protein-coupled receptor responsive to bile acids. The Journal of biological chemistry 278, 9435-9440, doi:10.1074/jbc.M209706200 (2003).

150 Jones, B. V., Begley, M., Hill, C., Gahan, C. G. \& Marchesi, J. R. Functional and comparative metagenomic analysis of bile salt hydrolase activity in the human gut microbiome. Proceedings of the National Academy of Sciences of the United States of America 105, 13580-13585, doi:10.1073/pnas.0804437105 (2008).

151 Lorenzo-Zuniga, V. et al. Oral bile acids reduce bacterial overgrowth, bacterial translocation, and endotoxemia in cirrhotic rats. Hepatology (Baltimore, Md.) 37, 551-557, doi:10.1053/jhep.2003.50116 (2003).

152 Langille, M. G. et al. Predictive functional profiling of microbial communities using $16 \mathrm{~S}$ rRNA marker gene sequences. Nature biotechnology 31, 814-821, doi:10.1038/nbt.2676 (2013).

153 Kanehisa, M., Furumichi, M., Tanabe, M., Sato, Y. \& Morishima, K. KEGG: new perspectives on genomes, pathways, diseases and drugs. Nucleic acids research 45, D353-d361, doi:10.1093/nar/gkw1092 (2017).

154 Kanehisa, M. \& Goto, S. KEGG: kyoto encyclopedia of genes and genomes. Nucleic acids research 28, 27-30 (2000).

155 Kanehisa, M., Sato, Y., Kawashima, M., Furumichi, M. \& Tanabe, M. KEGG as a reference resource for gene and protein annotation. Nucleic acids research 44, D457-462, doi:10.1093/nar/gkv1070 (2016).

156 Milner, D. A. et al. Quantitative Assessment of Multiorgan Sequestration of Parasites in Fatal Pediatric Cerebral Malaria. The Journal of infectious diseases 212, 1317-1321, doi:10.1093/infdis/jiv205 (2015).

157 Begley, M., Hill, C. \& Gahan, C. G. Bile salt hydrolase activity in probiotics. Applied and environmental microbiology 72, 1729-1738, doi:10.1128/aem.72.3.1729-1738.2006 (2006).

158 Maruyama, T. et al. Identification of membrane-type receptor for bile acids (M-BAR). Biochemical and Biophysical Research Communications 298, 714-719, doi:https://doi.org/10.1016/S0006-291X(02)02550-0 (2002). 
159 Yusta, B. et al. GLP-1R Agonists Modulate Enteric Immune Responses Through the Intestinal Intraepithelial Lymphocyte GLP-1R. Diabetes 64, 2537-2549, doi:10.2337/db14-1577 (2015).

160 Chong, J. et al. MetaboAnalyst 4.0: towards more transparent and integrative metabolomics analysis. Nucleic acids research, doi:10.1093/nar/gky310 (2018).

161 Xia, J., Psychogios, N., Young, N. \& Wishart, D. S. MetaboAnalyst: a web server for metabolomic data analysis and interpretation. Nucleic acids research 37, W652-660, doi:10.1093/nar/gkp356 (2009).

162 Xia, J., Sinelnikov, I. V., Han, B. \& Wishart, D. S. MetaboAnalyst 3.0-making metabolomics more meaningful. Nucleic acids research 43, W251257, doi:10.1093/nar/gkv380 (2015).

163 Xia, J. \& Wishart, D. S. MSEA: a web-based tool to identify biologically meaningful patterns in quantitative metabolomic data. Nucleic acids research 38, W71-77, doi:10.1093/nar/gkq329 (2010).

164 Comeau, A. M., Douglas, G. M. \& Langille, M. G. I. Microbiome Helper: a Custom and Streamlined Workflow for Microbiome Research. mSystems 2, doi:10.1128/mSystems.00127-16 (2017).

165 Andrews, S. FastQC: a quality control tool for high throughput sequence data. (2010).

166 Zhang, J., Kobert, K., Flouri, T. \& Stamatakis, A. PEAR: a fast and accurate Illumina Paired-End reAd mergeR. Bioinformatics (Oxford, England) 30, 614-620, doi:10.1093/bioinformatics/btt593 (2014).

167 Bushnell, B. BBMap: short read aligner. (2014).

168 Hannon, G. FASTX Toolkit: Sequence Read Preprocessing Software. (2014).

169 Rognes, T., Flouri, T., Nichols, B., Quince, C. \& Mahe, F. VSEARCH: a versatile open source tool for metagenomics. PeerJ 4, e2584, doi:10.7717/peerj.2584 (2016).

170 Caporaso, J. G. et al. QIIME allows analysis of high-throughput community sequencing data. Nature methods 7, 335-336, doi:10.1038/nmeth.f.303 (2010).

171 Kopylova, E., Noé, L. \& Touzet, H. SortMeRNA: fast and accurate filtering of ribosomal RNAs in metatranscriptomic data. Bioinformatics (Oxford, England) 28, 3211-3217, doi:10.1093/bioinformatics/bts611 (2012).

172 Mercier, C., Boyer, F., Bonin, A. \& Coissac, E. (2013).

173 Caporaso, J. G. et al. PyNAST: a flexible tool for aligning sequences to a template alignment. Bioinformatics (Oxford, England) 26, 266-267, doi:10.1093/bioinformatics/btp636 (2010).

174 DeSantis, T. Z. et al. Greengenes, a chimera-checked 16S rRNA gene database and workbench compatible with ARB. Applied and environmental microbiology 72, 5069-5072, doi:10.1128/aem.03006-05 (2006). 
175 Schriefer, A. E. et al. A multi-amplicon 16S rRNA sequencing and analysis method for improved taxonomic profiling of bacterial communities. Journal of microbiological methods 154, 6-13, doi:10.1016/j.mimet.2018.09.019 (2018).

176 Noedl, H., Socheat, D. \& Satimai, W. Artemisinin-resistant malaria in Asia. The New England journal of medicine 361, 540-541, doi:10.1056/NEJMc0900231 (2009).

177 Douglas, N. M., Anstey, N. M., Angus, B. J., Nosten, F. \& Price, R. N. Artemisinin combination therapy for vivax malaria. The Lancet. Infectious diseases 10, 405-416, doi:10.1016/s1473-3099(10)70079-7 (2010).

178 White, N. J. The treatment of malaria. The New England journal of medicine 335, 800-806, doi:10.1056/nejm199609123351107 (1996).

179 Lin, L., Mao, X., Sun, Y. \& Cui, H. Antibacterial mechanism of artemisinin / beta-cyclodextrins against methicillin-resistant Staphylococcus aureus (MRSA). Microbial Pathogenesis 118, 66-73, doi:https://doi.org/10.1016/j.micpath.2018.03.014 (2018).

180 Zhao, X. et al. Antimalarial agent artesunate protects Concanavalin Ainduced autoimmune hepatitis in mice by inhibiting inflammatory responses. Chemico-Biological Interactions 274, 116-123, doi:https://doi.org/10.1016/j.cbi.2017.07.012 (2017).

181 Kuang, M. et al. Artesunate Attenuates Pro-Inflammatory Cytokine Release from Macrophages by Inhibiting TLR4-Mediated Autophagic Activation via the TRAF6-Beclin1-PI3KC3 Pathway. Cellular physiology and biochemistry : international journal of experimental cellular physiology, biochemistry, and pharmacology 47, 475-488, doi:10.1159/000489982 (2018). 


\section{APPENDIX}

As the material in Chapter 2 was previously published, permission was sought and obtained from the journal Proceedings of the National Academy of the Sciences, documented below.

Wednesday, September 26, 2018 at 11:16:25 AM Eastern Daylight Time

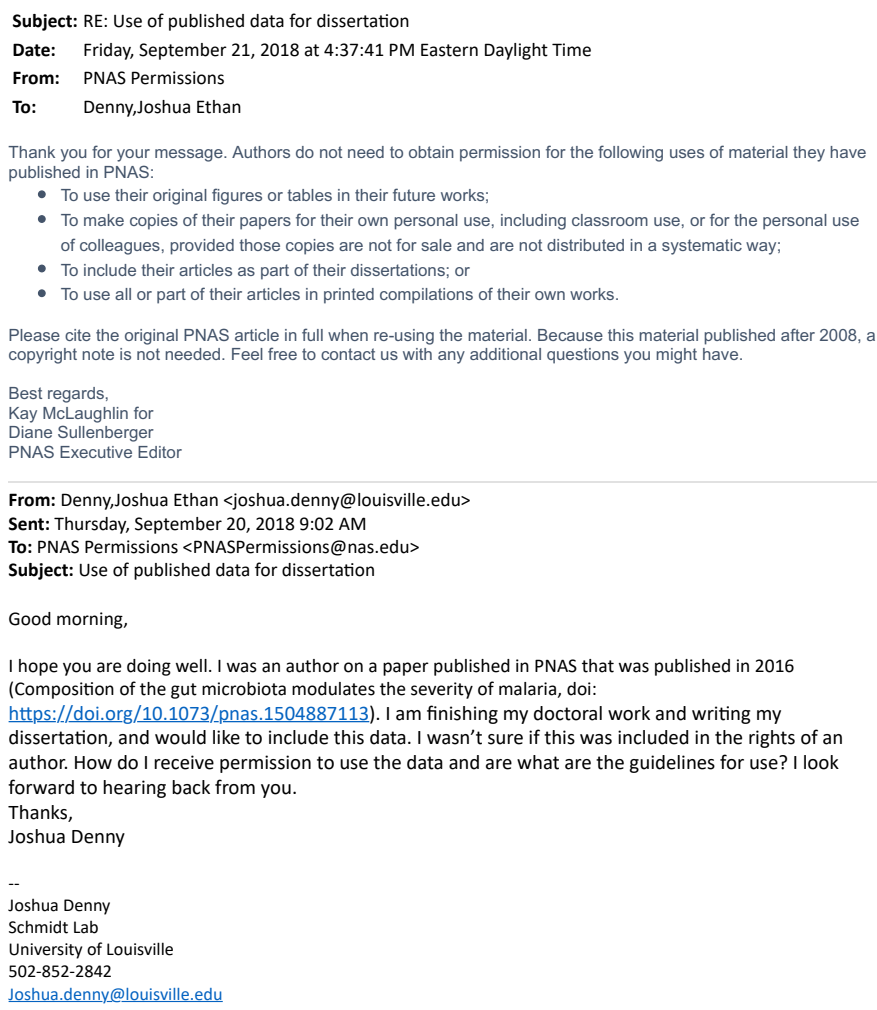




\title{
CURRICULUM VITAE
}

\author{
Joshua E. Denny \\ 1604 Hunnington Place, Apt. 12 \\ Louisville, KY 40220 \\ 931-434-2118 \\ Joshua.denny@louisville.edu
}

\section{Education}

2014 - Present

University of Louisville, Louisville, KY

Doctoral Student

Advisor: Nathan W. Schmidt, PhD

$2013-2014$

University of Tennessee, Knoxville, TN

Doctoral Student

Advisor: Nathan W. Schmidt, PhD

$2009-2013$

Carson-Newman University, Jefferson City, TN

Bachelor of Science in Biochemistry and Biology

\section{Research}

\section{3-Present}

Doctoral Research

University of Louisville, Louisville, KY

University of Tennessee, Knoxville, TN

Characterizing the effect of severe malaria on mouse gut homeostasis as well as the effects of antimalarial treatments on the microbiota.

2012-2013

Honors Thesis: LESD as a Practical Tool for Protein Structure Determination Carson-Newman University, Jefferson City, TN

Studied protein folding using an electrospray ionization-mass spectrometrybased labeling method, labeling electrospray deposition (LESD). 
2012

RUI: The Interdependence of Nutrient and Pheromone Sensing Pathways in Yeast

Maryville College, Maryville, TN

Used molecular biology techniques to study the impact of glucose levels on mating in Saccharomyces cerevisiae.

\section{Honors and Awards}

2015 Keystone Symposia Scholarship Recipient

2017 Gordon Conference: Red Blood Cells - Oral presentation

\section{Publications}

Joshua E. Denny and Nathan W. Schmidt. 2018. Oral Administration of Clinically Relevant Antimalarial Drugs Does Not Modify the Murine Gut Microbiota. Scientific Reports. Under Review.

Joshua E. Denny, Joshua B. Powers, Hector F. Castro, Jingwen Zhang, Swati Joshi-Barve, Shawn R. Campagna, and Nathan W. Schmidt. 2018. Severity of Plasmodium Infection in Mice Differentially Affects Gut-Liver Axis Homeostasis. Scientific Reports. Under Review.

Matthew M. Ippolito, Joshua E. Denny, Charles Langelier, Cynthia L. Sears, and Nathan W. Schmidt. 2018. Malaria and the microbiome: a systematic review. Clinical Infectious Diseases. ciy374.

Joshua E. Denny ${ }^{*}$, Whitney L. Powell*, and Nathan W. Schmidt. 2016. Local and Long-Distance Calling: Conversations between the Gut Microbiota and Intraand Extra-Gastrointestinal Tract Infections. Front Cell Infect Microbiol. 6.

${ }^{*}$ Co-first authors

Nicolas F. Villarino*, Gary R. Lecleir ${ }^{\star}$, Joshua E. Denny ${ }^{\star}$, Stephen P. Dearth, Christopher L. Harding, Sarah S. Sloan, Jennifer L. Gribble, Shawn R. Campagna, Steven W. Wilhelm, and Nathan W. Schmidt. 2016. Composition of the gut microbiota modulates the severity of malaria. Proc Natl Acad Sci. 113(8):2235:2240.

${ }^{*}$ Co-first authors 
Nicolas F. Villarino, Joshua E. Denny, and Nathan W. Schmidt. 2015.

Antimalarial Activity of Tulathromycin in a Murine Model of Malaria. Antimicrob. Agents Chemother. 59(6):3672-3674.

D. Grant Willhite, Jennifer R. Brigati, Katie E. Selcer, Joshua E. Denny, Zachary A. Duck, and Stephen E. Wright. 2014. Pheromone Responsiveness is regulated by components of the Gpr1p-mediated glucose sensing pathway in Saccharomyces cerevisiae. Yeast. 31:361-374.

\section{Presentations and Posters}

Denny, Joshua E., Josh Powers, Shawn R. Campagna, and Nathan W. Schmidt. Severity of Plasmodium Infection Differentially Affects Host Gut Homeostasis. Poster. Southeastern Immunology Symposium, June 16-17, 2018.

Denny, Joshua E., Josh Powers, Shawn R. Campagna, and Nathan W. Schmidt. Crosstalk Between the Gut Microbiota and Blood Stage Malaria. Poster. Research!Louisville, September 11-15, 2017.

Denny, Joshua E., Josh Powers, Shawn R. Campagna, and Nathan W. Schmidt. Crosstalk Between the Gut Microbiota and Blood Stage Malaria. Short Talk. Red Blood Cells: Formation, Genetics, Physiology and Disease Mechanisms, Newport, RI, July 16-21, 2017.

Villarino, Nicolas F., Gary R. Lecleir, Joshua E. Denny, Stephen P. Dearth, Christopher L. Harding, Sarah S. Sloan, Jennifer L. Gribble, Shawn R. Campagna, Steven W. Wilhelm, and Nathan W. Schmidt. Composition of the gut microbiota modulates the severity of malaria. Poster. Autumn Immunology Conference, Chicago, IL, November 20-23, 2015.

Villarino, Nicolas, Gary R. Lecleir, Joshua E. Denny, Stephen P. Dearth, Sarah S. Sloan, Jennifer L. Gribble, Shawn R. Campagna, Steven W. Wilhelm, and Nathan W. Schmidt. The Gut Microbiota Modulates the Severity of Malaria. Poster. Gut Microbiota Modulation of Host Physiology: The Search for Mechanism, Keystone, CO, March 1-6, 2015.

Nicolas Villarino, Joshua E. Denny, and Nathan W. Schmidt. Antimalarial Activity of Tulathromycin in a Murine Model of Malaria. Poster. Southeastern Immunology Symposium, Atlanta, GA, June 6-8, 2014.

Joshua E. Denny and Paul A. Martino. Probing the Topography of Rigid aConotoxin GI Using a Newly Proposed Mass Spectrometry-Based Covalent Labeling Method. Poster. Southeastern Regional Meeting of the American Chemical Society, Raleigh, NC, November 14-17, 2012. 


\section{Teaching Experience}

2017

Student Lecturer - University of Louisville

MBIO 610 - Research Methods in Microbiology and Immunology

$2013-2014$

Graduate Teaching Assistant - University of Tennessee

Biology 101 and General Biology

2012

Undergraduate Teaching Assistant - Carson-Newman University

Organic Chemistry Lab

\section{Technical Skills}

Mouse Handling - Oral gavage, intravenous injection, intraperitoneal injection, retro-orbital blood draw, organ/tissue extraction, isoflurane anesthesia Experimental Skills - Flow cytometry, DNA extraction, light microscopy, PCR, ELISA

Software - QIIME 1, PICRUSt 1, Graphpad Prism, FlowJo, Microsoft Office, Adobe Illustrator

\section{References}

Dr. Nathan Schmidt

Department of Microbiology and Immunology

University of Louisville

505 S. Hancock St.

Louisville, KY 40202

502-852-3741

Nathan.schmidt@louisville.edu

Dr. Michele Kosiewicz

Department of Microbiology and Immunology

University of Louisville

505 S. Hancock St.

Louisville, KY 40202

502-852-5343

Michele.kosiewicz@louisville.edu 


\author{
Dr. Nejat Egilmez \\ Department of Microbiology and Immunology \\ University of Louisville \\ 505 S. Hancock St. \\ Louisville, KY 40202 \\ 502-852-3539 \\ Nejat.egilmez@louisville.edu
}

